\title{
Prognostics and Health Management in Nuclear Power Plants: A Review of Technologies and Applications
}

JB Coble

P Ramuhalli

LJ Bond

JW Hines

BR Upadhyaya

July 2012

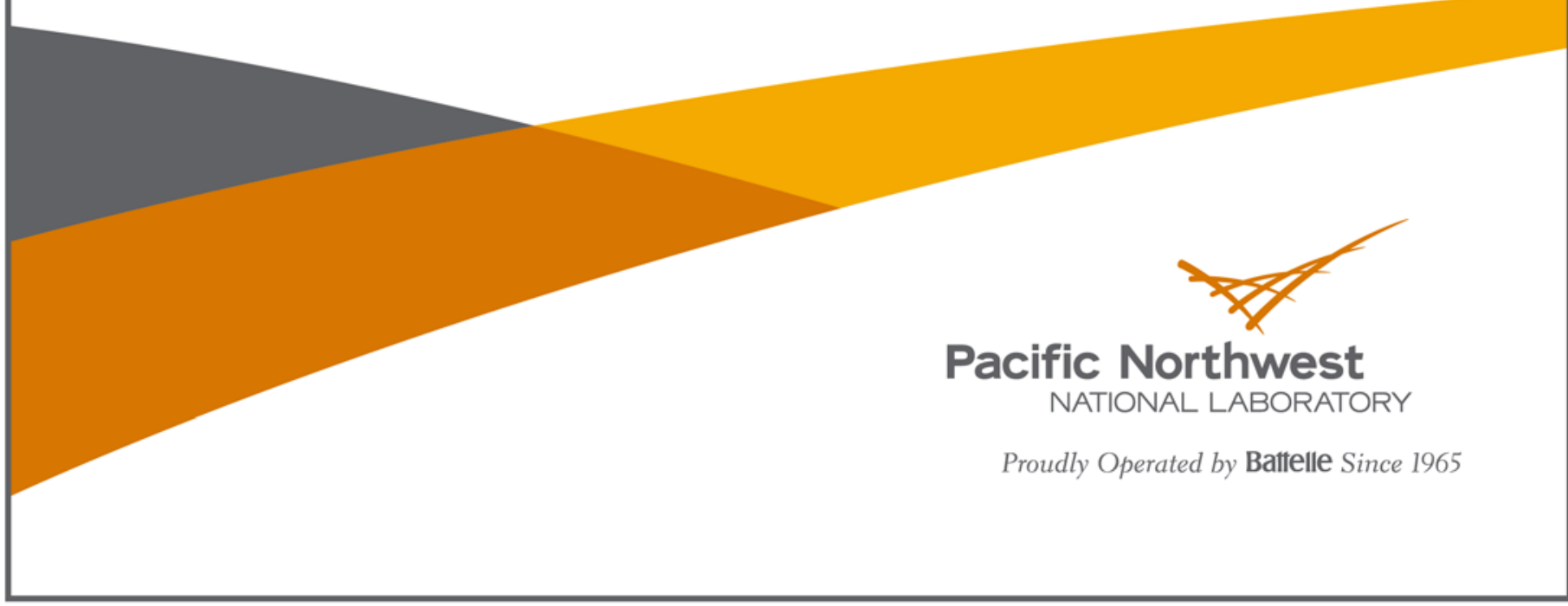




\title{
DISCLAIMER
}

This report was prepared as an account of work sponsored by an agency of the United States Government. Neither the United States Government nor any agency thereof, nor Battelle Memorial Institute, nor any of their employees, makes any warranty, express or implied, or assumes any legal liability or responsibility for the accuracy, completeness, or usefulness of any information, apparatus, product, or process disclosed, or represents that its use would not infringe privately owned rights. Reference herein to any specific commercial product, process, or service by trade name, trademark, manufacturer, or otherwise does not necessarily constitute or imply its endorsement, recommendation, or favoring by the United States Government or any agency thereof, or Battelle Memorial Institute. The views and opinions of authors expressed herein do not necessarily state or reflect those of the United States Government or any agency thereof.

\author{
PACIFIC NORTHWEST NATIONAL LABORATORY \\ operated by \\ BATTELLE \\ for the \\ UNITED STATES DEPARTMENT OF ENERGY \\ under Contract DE-AC05-76RL01830
}

Printed in the United States of America

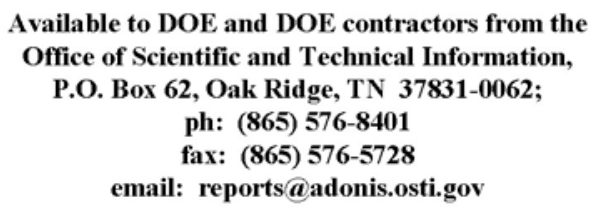

Available to the public from the National Technical Information Service 5301 Shawnee Rd., Alexandria, VA 22312 ph: (800) 553-NTIS (6847) email: orders@ntis.gov $<$ http://www.ntis.gov/about/form.aspx > Online ordering: http://www.ntis.gov 


\section{Prognostics and Health Management in Nuclear Power Plants: A Review of Technologies and Applications}

JB Coble

P Ramuhalli

LJ Bond $^{(\text {a) }}$

JW Hines ${ }^{(b)}$

BR Upadhyaya ${ }^{(b)}$

July 2012

Prepared for

the U.S. Department of Energy

under Contract DE-AC05-76RL01830

Pacific Northwest National Laboratory

Richland, Washington 99352

(a) Formerly Lab Fellow at PNNL

Currently Director of CNDE at Iowa State University Ames, Iowa 50011

(b) The University of Tennessee

Knoxville, Tennessee 37996 



\section{Abstract}

Recent years have seen a resurgence of nuclear power worldwide, with interest in extending the operating life of the approximately 436 reactors currently in service (as of March, 2012), 61 new reactors being constructed, and as many as 162 under consideration. Renewed worldwide interest in nuclear power has been somewhat tempered by the March 2011 incident at Fukushima Dai-ichi in Japan. However, nuclear power is still considered a key element in meeting future worldwide sustainable energy, energy security, and emissions goals. Currently, three separate thrusts to safe and economical nuclear power development for energy security are being pursued in the United States: (i) longer term operation for the legacy fleet, from 40-60 and possibly 60-80 years; (ii) near-term new nuclear plants with a 60-year design life; and (iii) small modular reactors, which are expected to employ light water reactor technology at least in the medium term. Within these activities, attention is turning to enhanced methods for plant component and structural health management.

The operating U.S. fleet includes 104 light water reactors. In addition, there are now (as of May 2012) four new nuclear power plants (AP-1000 plants) under construction in the United States, and two delayed plants are being completed by the Tennessee Valley Authority. There is also interest in the United States in small modular reactors (SMRs), which could be easier to match to existing grid infrastructure and which could replace aging coal fired plants. The current low price for natural gas presents a challenge to the economics of nuclear power, at least in the short term; however, some recent studies have demonstrated that nuclear generation will be competitive in the longer term (at least in some markets) when anticipated escalation in gas prices and the cost of building, operating, and maintaining gas-fired plants are considered over those same time periods.

This report reviews the current state of the art of prognostics and health management (PHM) for nuclear power systems and related technology currently applied in field or under development in other technological application areas, as well as key research needs and technical gaps for increased use of PHM in nuclear power systems. The historical approach to monitoring and maintenance in nuclear power plants (NPPs), including the Maintenance Rule for active components and Aging Management Plans for passive components, are reviewed. An outline is given for the technical and economic challenges that make PHM attractive for both legacy plants through Light Water Reactor Sustainability (LWRS) and new plant designs. There is a general introduction to PHM systems for monitoring, fault detection and diagnostics, and prognostics in other, non-nuclear fields. The state of the art for health monitoring in nuclear power systems is reviewed. A discussion of related technologies that support the application of PHM systems in NPPs, including digital instrumentation and control systems, wired and wireless sensor technology, and PHM software architectures is provided. Appropriate codes and standards for PHM are discussed, along with a description of the ongoing work in developing additional necessary standards. Finally, an outline of key research needs and opportunities that must be addressed in order to support the application of PHM in legacy and new NPPs is presented. 



\section{Summary}

Recent years have seen a resurgence of nuclear power worldwide, with interest in extending the operating life of the approximately 436 reactors currently in service (as of March 2012), 61 new reactors being constructed, and as many as 162 under consideration. While the renewed interest in nuclear power has been somewhat tempered by the March 2011 incident at Fukushima Dai-ichi in Japan, nuclear power is still considered key to meeting future worldwide sustainable energy, energy security, and emissions goals. Currently, three separate thrusts to safe and economical nuclear power development for energy security are being pursued in the United States.

The first thrust is focused on life extension of operating light-water reactors (LWRs), referred to as long-term operation (LTO). In the United States, 71 reactors have been approved for license extension from 40 years to 60 years of operation, and 9 plants have already entered LTO (in some cases, with power uprates). Similar trends are being reported worldwide. Interest is now turning to a second license extension to enable operation from 60-80 years, so-called extended LTO or life-beyond-60 (LB60). The question of safely and economically operating some of these plants beyond 60 years is now being considered in anticipation of this second round of license extensions. The existing fleet is considered to be too valuable to decommission, even if $\$ 1 \mathrm{~B}$ per plant is needed for refurbishment to give another 20 years of operation. Through power uprates of existing plants, U.S electric companies have added the equivalent of five or six new reactors in the past 30 years by upgrading legacy plants in order to produce more power from the same plant (Carter 2006).

The second pathway focuses on new LWR reactor designs. In the United States, the Westinghouse AP-1000 has emerged as the primary Generation III+ design, and four plants are currently under construction. The AP-1000 has enhanced safety features and a 60-year design life. Similar features are being included in other Generation III+ designs, including the ABWR, APWR, EPR, EBR, and Advanced CANDU Reactor (ACR), many of which are being built worldwide.

The third thrust is in the area of advanced reactor designs that rely on passive safety systems for decay heat removal; in the near-term, there is a focus on light water reactor designs and a particular focus on so-called small modular reactors (SMRs).

Over the years, operational experience has shown that greater situational awareness of the state of safety-critical nuclear plant systems, structures, and components is necessary, particularly as they age over time due to exposure to harsh service conditions. While replacement of a subset of components is possible, it may be economically prohibitive to replace several of the larger components, including the reactor pressure vessel and primary piping. Thus, detection, management, and mitigation of aging-related degradation in these critical components become important to maintain safety margins. In this context, the technical challenges related to detecting, characterizing, monitoring, and managing materials degradation need to be identified and addressed. These challenges are not unique to the operating fleet of LWRs, as the next generation of nuclear power reactors is expected to have similar needs in terms of managing and mitigating degradation; therefore, it is likely that any technology developed for detecting and characterizing degradation will have applications beyond the current fleet. Additionally, some nextgeneration reactor designs have potentially increased monitoring needs due to extended fuel cycles, reduced access to critical components, and remote siting with reduced maintenance staff. 
The key technology developments necessary for detecting and managing degradation in reactor components are: (1) nondestructive measurement methods and analyses to detect degradation and anomalies, (2) algorithms to characterize and monitor the degradation state of the component, and (3) algorithms that use the degradation state information to determine remaining useful life (RUL) and probability of failure (POF) of the component. The POF information may then be used in a probabilistic risk assessment (PRA) model to assess the risk significance of the degradation and the corresponding reduced safety margin. Together, these technologies constitute prognostics and health management (PHM) systems. Ideally, degradation detection should occur early in the degradation development lifecycle, to enable the application of appropriate mitigation or repair actions, thereby maintaining the necessary safety margins. Appropriate PHM systems therefore can potentially preclude serious consequences due to aging-related faults.

This report reviews the current state of the art of prognostics and health management for nuclear power systems and related technology currently applied in field or under development in other technological application areas.

The nuclear power industry worldwide poses a unique challenge for application of health management systems. NPPs worldwide are closely regulated by national and international licensing bodies, and application of PHM systems which may impact safety-critical systems (and some non-safetyrelated systems) will require a clear licensing basis outlining appropriate algorithms, architectures, and applications. In the United States, the evaluation of such a licensing basis is the responsibility of the Nuclear Regulatory Commission.

Section 2 describes the historical approach to monitoring and maintenance in nuclear power plants (NPPs), including the Maintenance Rule for active components and Aging Management Plans for passive components. The Maintenance Rule, enacted in the mid-1990s, provides a performance-based approach to monitoring and improving the effectiveness of active component maintenance; however, it does not encourage efficient (or discourage inefficient) maintenance practices. Passive components do not fall under the purview of the Maintenance Rule, but are managed by periodic in-service inspection as dictated by the Aging Management Plan. While these approaches have been adequate for maintaining safety margins in the past, several challenges exist that suggest the move to more predictive monitoring and maintenance activities.

Section 3 outlines the technical and economic challenges that make PHM attractive for both legacy plants through Light Water Reactor Sustainability (LWRS) and new plant designs. While the traditional approaches to maintenance and degradation management (the Maintenance Rule and Aging Management Plan) have been adequate in the past, these approaches are generally not optimized in terms of effort, time, or cost. As plants move into longer service lives (both through LWRS and extended license periods of newer designs), the frequency of periodic inspection and maintenance may have to increase to compensate for increasing failure rates of known fault modes and heretofore unknown fault modes. Advanced reactor designs introduce an additional set of challenges with exposure to potentially more severe stressors and the use of new, innovative materials to accommodate them. These technical challenges, along with the economic benefits of moving from periodic and find-and-fix maintenance strategies to just-in-time maintenance, build a strong case for applying PHM in current and future NPPs. 
This is followed by a general introduction to PHM systems in Section 4. Full PHM systems typically include several tasks or modules, including data collection, fault detection, fault diagnostics, system prognostics, and planning. By applying this entire suite, the goals of most PHM systems can be realized: increased assurance of safety; increased productivity; reduced downtime; reduced number and severity of failures, particularly unanticipated failures; optimized operating performance; extended operating periods between maintenance; reduced unnecessary planned maintenance; and reduced life-cycle cost. Section 4 discusses the current state of the art in data collection, monitoring and fault detection, diagnostics, and prognostics for both passive and active components. In some cases, such as data collection and fault detection, the algorithms and approaches for active and passive components are different, but the overarching approach to PHM is shared.

Current technologies for monitoring, fault detection and diagnostics, and prognostics in other, nonnuclear fields are discussed in Section 5. This discussion focuses on advances in health monitoring for electronics, defense applications, avionics, and wind turbines. While the specific models and results found in these areas may not be directly applicable to NPPs, the algorithms and approaches generally are applicable.

Section 6 outlines the state of the art for health monitoring in nuclear power systems. This discussion is divided between the advances in active components (focusing on sensors, pumps, and valves) and passive components (e.g., metals, reactor vessel internals, concrete, cables, buried pipes, and transformers). The state of the art is largely focused on fault detection and diagnostics for these components, although some work in prognostics is described. Additionally, the results of pilot applications and fielded systems in NPPs are summarized.

Section 7 describes related technologies that support the application of PHM systems in NPPs, including digital instrumentation and control (I\&C) systems, wired and wireless sensor technology, and PHM software architectures.

Appropriate codes and standards for PHM are discussed in Section 8, along with a description of the ongoing work in developing additional necessary standards. The applicable codes include those which define the NDE techniques approved for use in NPPs (defined by the ASME Boiler and Pressure Vessel Code), as well as standards developed for prognostics and health monitoring in general. These algorithmic standards are not application-specific and have not been reviewed by the U.S. Nuclear Regulatory Commission (NRC) for application to U.S. NPPs. It will likely be necessary to develop nuclear-specific and/or nuclear-applicable standards for advanced NDE and PHM techniques for review and endorsement by the NRC.

Section 9 outlines key research needs and opportunities that must be addressed in order to support the application of PHM in legacy and new NPPs. While significant advances have been made in the last 20 years, much work is still needed to bring PHM from the research arena into NPP deployment. Some of these needs are specific to the application to NPPs and nuclear components, including high-fidelity physics-of-failure models, experimental failure and aging data, and optimal sensor design and placement (particularly for passive components) to give adequate coverage at reasonable cost. Some areas of research are more general to the PHM community as a whole (uncertainty analysis and propagation, online performance metrics, verification and validation of PHM algorithms and models, etc.), and ongoing research in other fields can likely be leveraged to address these needs for NPPs. Finally, welldefined methods for incorporating the results of PHM algorithms into a more holistic view of plant 
operation, maintenance, and decision making are needed to provide a practical advantage for the use of PHM in terms of safety and economics.

Finally, concluding remarks are given in Section 10 and references in Section 11. 


\section{Acknowledgments}

The material presented in this report was developed from material gathered with support from PNNL's sustainable nuclear power initiative and DOE-NE FY 11 project funding under the light water

reactor sustainability program. The authors would like to extend their thanks to Kay Hass for her ongoing support, attention to detail, and technical editing expertise in preparing and finalizing this document. 



\section{Acronyms and Abbreviations}

\begin{tabular}{|c|c|}
\hline AAKR & Autoassociative Kernel Regression \\
\hline AANN & Autoassociative Neural Networks \\
\hline ABWR & advanced boiling water reactors \\
\hline $\mathrm{AE}$ & acoustic emission \\
\hline AMP & aging management plan \\
\hline AMS & Analysis and Measurement Services Corporation \\
\hline APR & advanced pattern recognition \\
\hline ASME & American Society of Mechanical Engineers \\
\hline ATO & Advanced Technology Office \\
\hline BIST & built-in self test \\
\hline BPV & Boiler \& Pressure Vessel \\
\hline BWR & boiling water reactor \\
\hline BWRVIP & Boiling Water Reactor Owners Group's Vessel and Internals Project \\
\hline CALCE & Center for Advanced Life-Cycle Engineering \\
\hline CASS & cast austenitic stainless steel \\
\hline CBA & cost-benefit analysis \\
\hline CBM & condition-based maintenance \\
\hline $\mathrm{CBM}+$ & condition-based maintenance plus \\
\hline $\mathrm{CDF}$ & cumulative density function \\
\hline CRP & Coordinated Research Programme \\
\hline $\mathrm{CSTH}$ & continuous system telemetry harness \\
\hline CUSUM & cumulative sum (chart) \\
\hline DARTS & diagnostic reasoning technology \\
\hline DGA & dissolved gas analysis \\
\hline DIC & digital image correlation \\
\hline DOE & U.S. Department of Energy \\
\hline $\mathrm{eCM}$ & equipment condition monitoring \\
\hline EPA & Environmental Protection Agency \\
\hline EPR & European Pressurized Water Reactor \\
\hline EPRI & Electric Power Research Institute \\
\hline EULM & Error Uncertainty Limit Monitoring \\
\hline FAMOS & Fatigue Monitoring System \\
\hline FCP & false call probability \\
\hline FMEA & failure modes and effects analysis \\
\hline
\end{tabular}


FMECA

FPK

GALL

GFR

GPM

GW

HFIR

HUMS

HVAC\&R

I\&C

IAEA

IASCC

ID

ISHM

ISI

IVHM

JSF

$\mathrm{kNN}$

LEAP

LFR

LIRA

LTO

LWR

LWRS

MAS

MOV

MRP

MSET

MSR

NDE

NESCC

NPAR

NPP

NRC

O\&M

OD

OLM

ORNL failure mode, effects, and criticality analysis

Fokker-Planck-Kolmorgorov

Generic Aging Lessons Learned

gas-cooled fast reactor

general path model

guided ultrasonic wave

High Flux Isotope Reactor

health and usage monitoring systems

heating, ventilating, air conditioning, and refrigeration

instrumentation and control

International Atomic Energy Agency

irradiation-assisted SCC

inner diameter

integrated structural health management

in-service inspection

integrated vehicle health management

Joint Strike Fighter

k-nearest neighbors

Life Extension Analysis and Prognostics

lead-cooled fast reactor

line resonance analysis

long-term operation

light-water reactor

Light Water Reactor Sustainability

multi-agent system

motor operated valves

Materials Reliability Program

Multivariate State Estimation Technique

molten salt reactor

nondestructive examination

Nuclear Energy Standards Coordination Collaborative

Nuclear Plant Aging Research

nuclear power plant

U.S. Nuclear Regulatory Commission

operations and maintenance

outer diameter

on-line monitoring

Oak Ridge National Laboratory 
PBMR

PCA

PD

PHM

PIT

PMBD

PNNL

POD

POF

PRA

progROC

PWR

PWROG

RADS

RCP

$\mathrm{RCV}$

REDI-PRO

RUL

SAFT

SBIR

SCADA

SCC

SCWR

SFR

SMR

SNR

SPRT

SSBF

SSC

TDR

TEDANN

TS

UHF

UML

USDI

$\mathrm{V} \& \mathrm{~V}$

VHM

VHTR
Pebble Bed Modular Reactor

principal component analysis

partial discharge

prognostics and health management

probability integral test

predictive maintenance basis database

Pacific Northwest National Laboratory

probability of detection

probability of failure

probabilistic risk assessment

prognostics extension to the receiver operating characteristics

pressurized water reactor

Pressurized Water Reactor Owner's Group

Reliability and Availability Data System

reactor coolant pump

reactor containment vessel

Real-time Engine Diagnostics-Prognostics

remaining useful life

synthetic aperture focusing technique

Small Business Innovation Research

Supervisory Control and Data Acquisition

stress corrosion cracking

super-critical water-cooled reactor

sodium-cooled fast reactor

small modular reactors

signal-to-noise ratio

sequential probability ratio test

simplified stress-based fatigue

systems, structures, and components

time domain reflectometry

Turbine Engine Diagnostics using Artificial Neural Networks

technical specifications

ultra-high frequency

Unified Modeling Language

U.S. Department of the Interior

verification and validation

vehicle health management

very high-temperature gas-cooled reactor 
WEC

$\overline{\mathrm{X}}-\mathrm{R}$

$\overline{\mathrm{X}}-\mathrm{S}$

YPG wind energy convertor

mean-range (control chart)

mean-standard deviation (control chart)

Yuma Proving Ground 


\section{Contents}

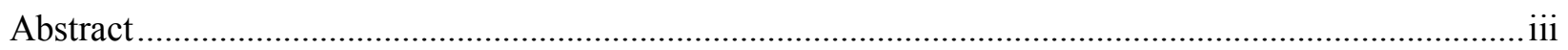

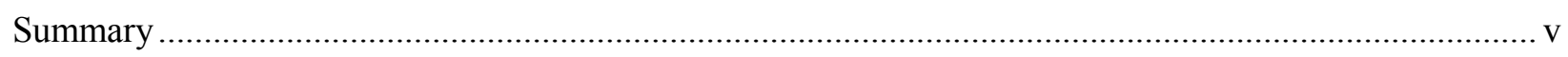

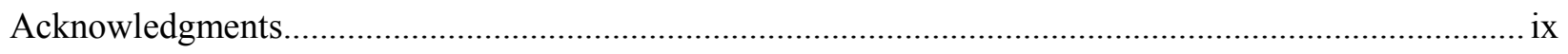

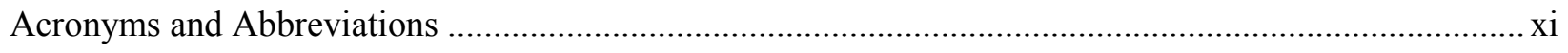

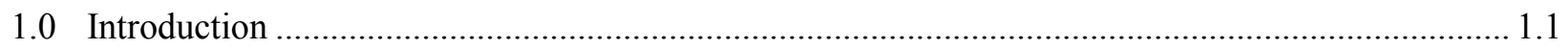

2.0 Historical Approach to Monitoring and Maintenance in NPPs in the USA .................................. 2.1

2.1 Active Components - the Maintenance Rule .................................................................... 2.1

2.2 Passive Components - Aging Management Plans ............................................................... 2.2

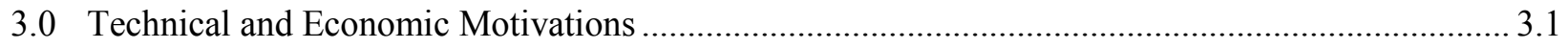

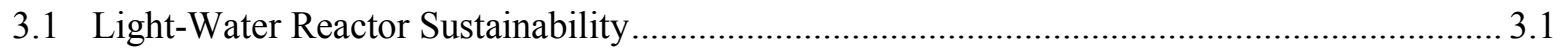

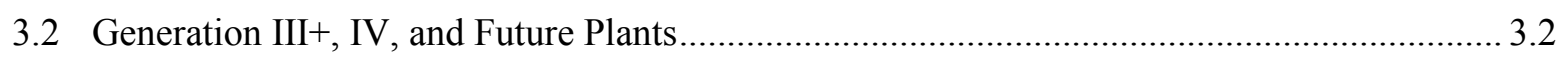

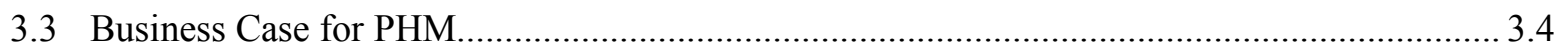

4.0 Introduction to Prognostics and Health Management Systems ..................................................... 4.1

4.1 Data Collection - NDE for Passives, Variable Sensing for Actives ....................................... 4.3

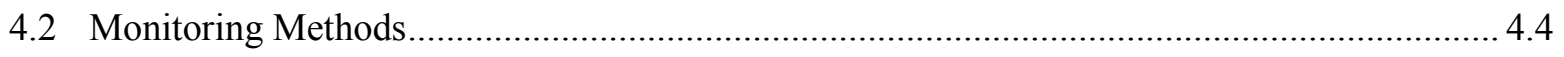

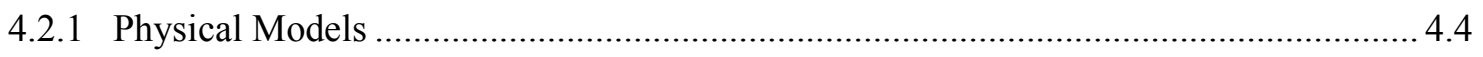

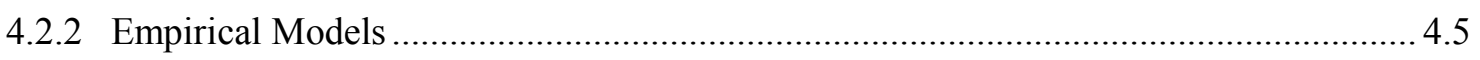

4.2.3 Fault Detection Methods ....................................................................................... 4.5

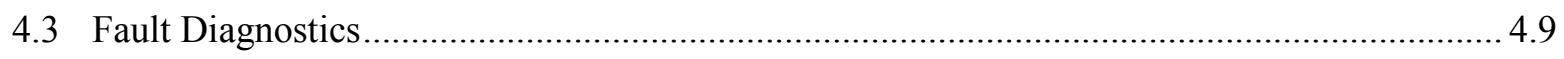

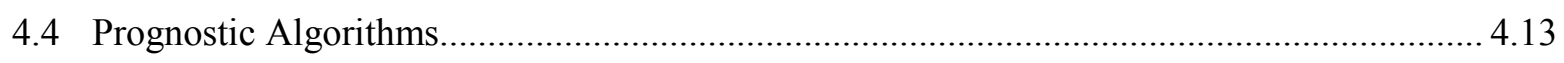

4.4.1 Prognostic Uncertainty Analysis ............................................................................ 4.14

4.4.2 Prognostic Performance Metrics ......................................................................... 4.16

5.0 Applications of Current Technologies in Other Fields ............................................................. 5.1

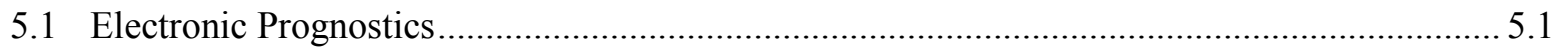

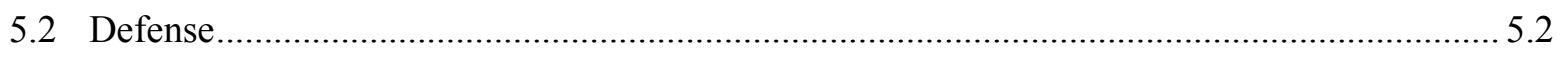

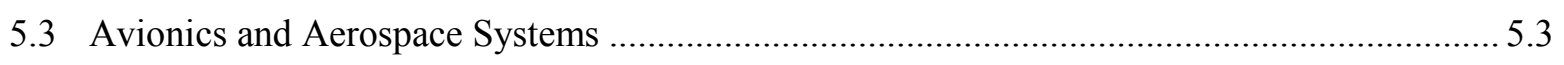

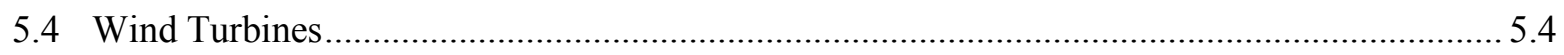

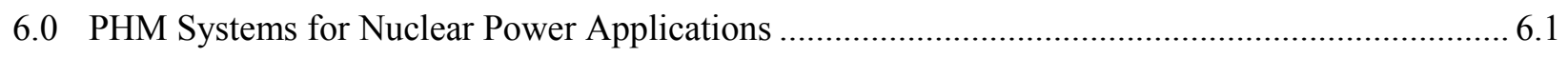

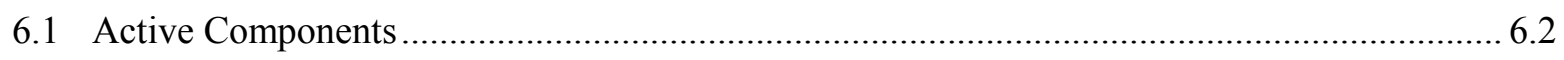

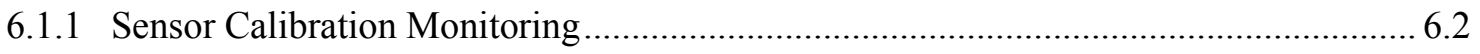

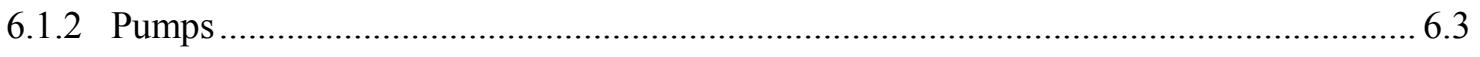

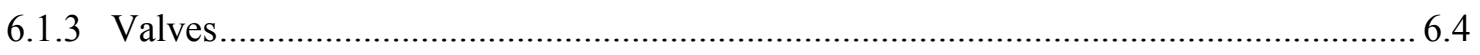

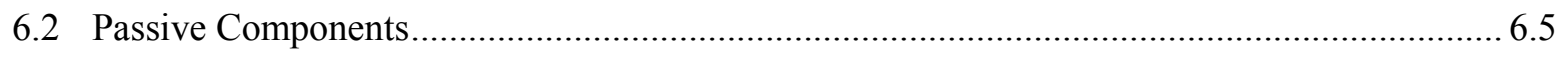

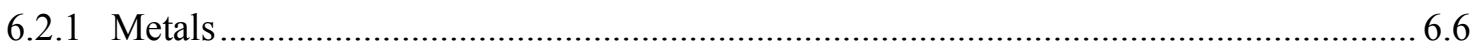

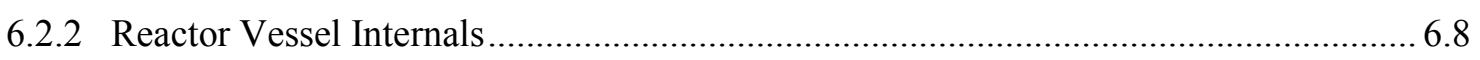

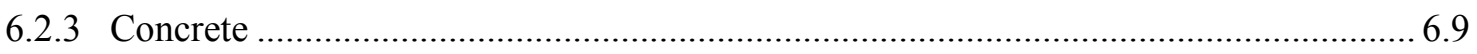




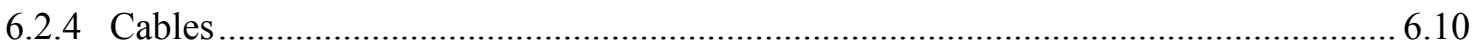

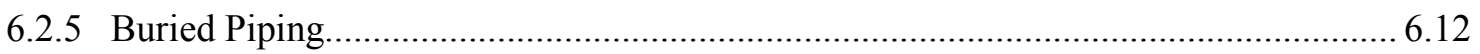

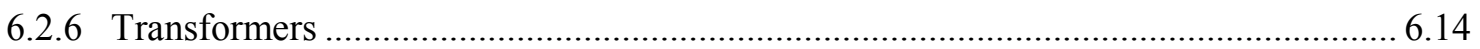

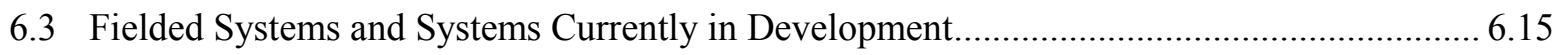

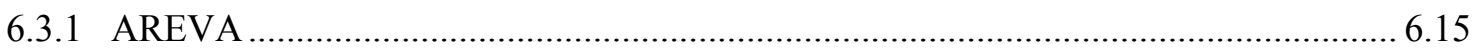

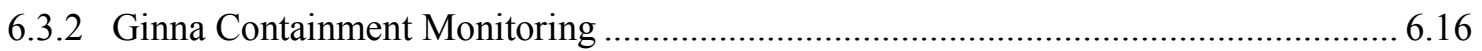

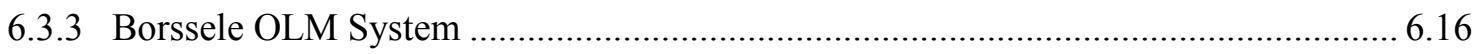

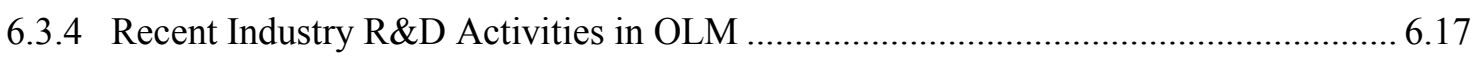

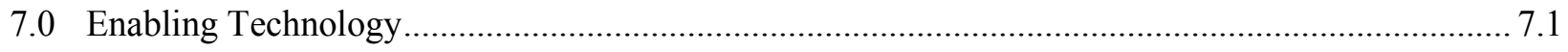

7.1 Digital Instrumentation and Control Systems ............................................................... 7.1

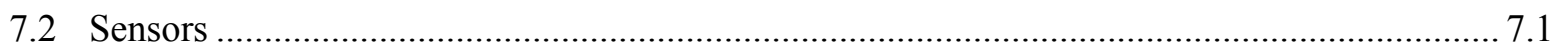

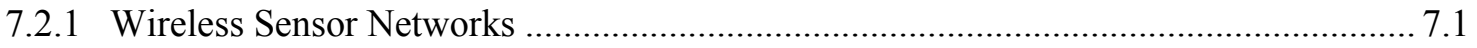

7.3 Monitoring the PHM Hardware and Validating Data ......................................................... 7.2

7.4 Prognostic Architectures (Software) ..................................................................................... 7.2

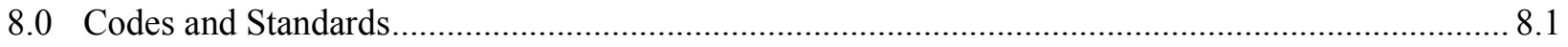

9.0 Research Needs and Opportunities to Support PHM in NPPs.................................................. 9.1

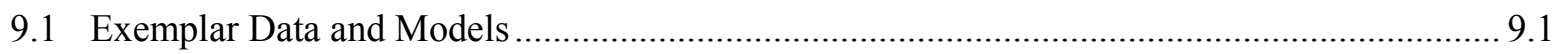

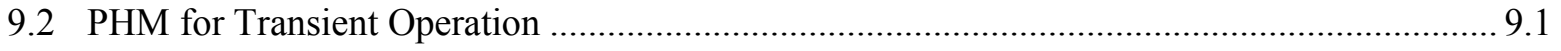

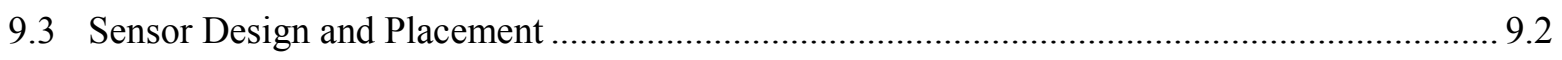

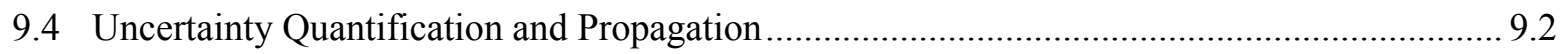

9.5 Online Prognostic Model Performance Assessment ........................................................... 9.3

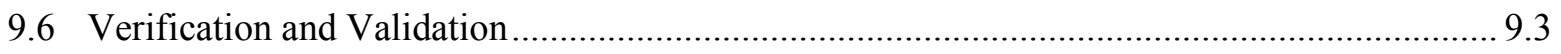

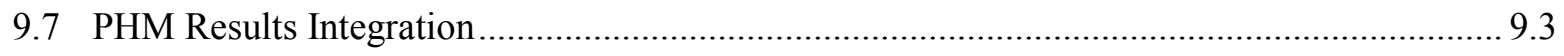

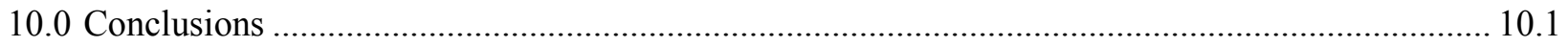

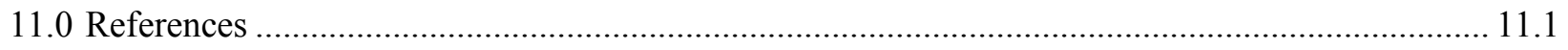




\section{Figures}

4.1 Suite of Modules in a Health Monitoring System …..................................................................... 4.2

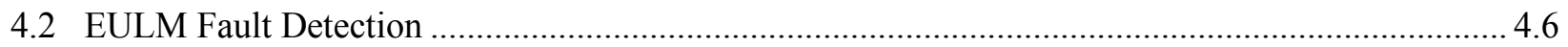

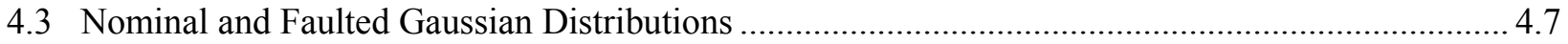

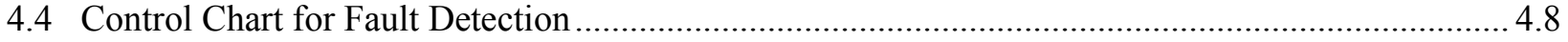

4.5 Prognostic Algorithm Categorization .......................................................................................... 4.13

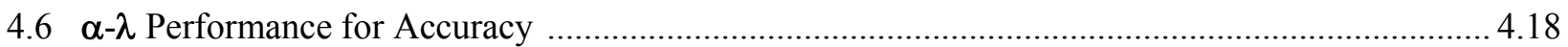

\section{Tables}

4.1 Assessment of State of Maturity for Diagnostic [D] and Prognostic [P] Technologies ................... 4.2

4.2 Categorization of Select Empirical State Estimation Methods ....................................................... 4.5

4.3 Classification of Selected Prognostic Algorithms ...................................................................... 4.15

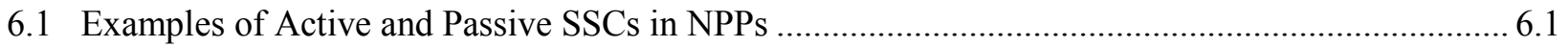

6.2 Summary of NDE Technologies Included in Section XI of the ASME Boiler and Pressure

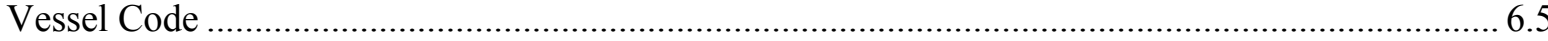





\subsection{Introduction}

Recent years have seen a resurgence of nuclear power worldwide, with interest in extending the operating life of the approximately 436 reactors currently in service (as of 2011), 61 new reactors being constructed, and as many as 162 under consideration. While the renewed interest in nuclear power has been somewhat tempered by the March 2011 incident at Fukushima Dai-ichi in Japan, nuclear power is still considered key to meeting future worldwide energy and emissions goals. Currently, three separate thrusts to support safe and economical nuclear power development for energy security are being pursued in the United States.

The first pathway is addressing life extension of operating light-water reactors (LWRs). In the United States, many of the operating 104 reactors are moving to extended operation (from the initial license period of 40 years to extended life of 60 years), and similar trends are being reported worldwide (Chockie et al. 1991; Bond 1999; Gregor and Chockie 2006; Bond et al. 2008c). The question of safely and economically operating some of these plants beyond 60 years is now being considered, because the cost of building replacement generating capacity while also building new plants to meet anticipated growth in electricity demand would challenge the available technical, manufacturing, regulatory, and economic infrastructures. The first round of license extensions required extensive research into aging mechanisms, detection, and management (Shah and MacDonald 1993; Tipping 2010). In the United States, this information was used by the NRC to develop the technical basis for license renewal that included the AMP and the Maintenance Rule (NRC 2001, 2005a, b, 2010d). Similar questions about aging detection and management are being asked for subsequent license extensions, with the anticipation being that other aging mechanisms will dominate as plants go beyond 60 years.

The second area focuses on new LWR reactor designs, commonly referred to as Generation III+ $($ Gen III + ) reactors. In the United States, the Westinghouse AP-1000 design has emerged as the primary Gen III+ design. This design includes enhanced safety features and a 60-year design life. Similar features are being included in other Gen III+ designs, including the ABWR, APWR, EPR, EBR, ACR, many of which are being built worldwide.

The third thrust is in the area of advanced reactor designs that rely on passive safety systems for decay heat removal (called Generation IV designs), with a particular focus on so-called small modular reactors (SMR) (Abu-Khader 2009; Ingersoll 2009). Gen IV and SMR designs for near-term deployment are expected to adapt well-known LWR technology; however, these reactors may include new design features, such as integral primary systems, and new components, such as helical coil heat exchangers. Longer-term designs are expected to feature non-light water coolants, including liquid metal, liquid salt, high-temperature gas, etc., and operate at higher temperatures than those seen in current LWR designs. These advanced designs will have additional monitoring needs due to high-temperature degradation phenomena in advanced materials, measurement challenges associated with extreme coolant environments, and unique operational characteristics. Both LWR-based and advanced designs of fullscale and small reactors will likely have additional monitoring needs that may not be met by traditional inspection techniques. Continuous surveillance of component condition is also a means to compensate for a relative lack of understanding of long-term component and materials behavior.

Operational experience (with the light water fleet in the United States and overseas, heavy water fleet worldwide, as well as advanced reactor designs that have been built and operated in limited numbers) has 
shown that greater situational awareness of the state of safety-critical nuclear plant systems, structures, and components is necessary, particularly as they age due to exposure to harsh service conditions. While replacement of a subset of components is possible, and may even be economically attractive, it may be economically prohibitive to replace several of the larger components, including the reactor pressure vessel, primary piping, and instrumentation cabling. Thus, management and mitigation of aging-related degradation in these critical passive components becomes important to maintain safety margins. In this context, the technical challenges related to detecting, characterizing, monitoring, and managing materials degradation need to be identified and addressed (Chockie et al. 1991; Bond et al. 2011a). These challenges are not unique to the operating fleet of LWRs, as the next generation of nuclear power reactors is expected to have similar requirements in terms of managing and mitigating degradation; therefore, it is likely that any technology developed for detecting and characterizing degradation will have applications beyond the current fleet.

The key technology developments necessary for detecting and managing degradation in reactor components are: (1) nondestructive measurement methods and analyses to detect degradation and anomalies; (2) algorithms to characterize and quantify degradation (with a degradation metric) and to monitor the degradation state of the component; and (3) algorithms that use the degradation state information, combined with expected operating conditions (stressors), to determine remaining useful life (RUL) of the component. The RUL information may then be applied to a probabilistic risk assessment (PRA) model to assess the risk significance of the degradation and the corresponding safety margin. Together, these technologies constitute prognostics and health management (PHM) systems. Ideally, degradation detection should occur early in the degradation development lifecycle, to enable the applicability of any appropriate mitigation actions (Stevenson 2006). Appropriate PHM systems can potentially preclude serious consequences due to aging-related faults.

This report reviews the current state of the art of prognostics and health management for nuclear power systems and related technology currently applied in field or under development in other technological application areas. Section 2 describes the historical approach to monitoring and maintenance in nuclear power plants, including the Maintenance Rule for active components and Aging Management Plans for passive components. Section 3 outlines the technical and economic challenges that make PHM attractive for both legacy plants through Light Water Reactor Sustainability (LWRS) and new plant designs. This is followed by a general introduction to PHM systems in Section 4. Current technologies for monitoring, fault detection and diagnostics, and prognostics in non-nuclear fields are discussed in Section 5. Section 6 outlines the state of the art for health monitoring in nuclear power systems. Section 7 describes related technologies that support the application of PHM systems in nuclear power plants (NPPs), including digital instrumentation and control (I\&C) systems, wired and wireless sensor technology, and PHM software architectures. Appropriate codes and standards for PHM are discussed in Section 8, along with a description of the ongoing work in developing additional necessary standards. Section 9 outlines key research needs and opportunities that must be addressed in order to support the application of PHM in legacy and new NPPs. Finally, concluding remarks are given in Section 10 and references in Section 11. 


\subsection{Historical Approach to Monitoring and Maintenance in NPPs in the USA}

The U.S. Nuclear Regulatory Commission (NRC) requires that commercial nuclear power plants (NPPs) operate in a safe condition. In looking at longer term operation, the NRC published its license renewal rule, Title 10 of the Code of Federal Regulations (10 CFR) Part 54, on May 8, 1995, which provides the requirements for renewal of operating licenses for nuclear power plants. This explicitly states in 10 CFR 54.21(a)(1)(i) that an aging management review of containment structures is performed to ensure that the effects of aging will be managed so that their intended functions will be maintained for the period of extended operation. In 1990, the Nuclear Management and Resources Council (NUMARC), now the Nuclear Energy Institute (NEI), submitted for NRC review, the industry reports (IRs), NUMARC Report 90-01 and NUMARC Report 90-10, addressing aging management issues associated with PWR containments and BWR containments for license renewal, respectively (Liu et al. 1997).

Ensuring that the intended functions of SSCs in an NPP will be maintained for the period of operation and extended operation is, in part, a fulfillment of the defense-in-depth safety strategy employed by the NRC. Defense-in-depth includes multiple, independent, and redundant layers of protection to mitigate and contain possible accident scenarios that may result in radiation release (Diaz 2004). Chockie et al. (1991) identified four elements that are necessary for effective maintenance and aging management programs:

1. Prioritized selection of critical systems, structures, and components;

2. Collection and analysis of equipment performance measures to understand aging;

3. Applying maintenance to mitigate the effects of aging; and

4. The use of feedback to improve the maintenance and aging management programs.

Monitoring and maintenance programs prescribed by the NRC and implemented at NPPs draw a distinction between active and passive SSCs. Simply put, passive SSCs do not move during normal functions while active SSCs do. Passive components include structures, pressure vessel, heat exchangers, cables, pipes, pressurizers, steam generators, etc. Active components include pumps, motors, generators, sensors, control rod drive, etc. The distinction between active and passive components can be complicated. For instance, pumps and valves are generally considered active components, but their bodies, casings, and support structures are passive. Historically, maintenance of active components is covered by the Maintenance Rule, while passive component degradation and maintenance are addressed under the plant's Aging Management Plan. These are essential elements of the NRC's defense-in-depth policy for ensuring the safety and integrity of operating reactors. The following sections briefly discuss the Maintenance Rule and Aging Management Plan, respectively.

\subsection{Active Components - the Maintenance Rule}

The requirements for performance monitoring and aging management of active components are contained in the Maintenance Rule (the Rule), defined in (10 CFR 50.65 2011); section (a) of the Rule defines the technical requirements while section (b) defines the scope of SSCs that fall into the Rule. The Rule was designed to provide the nuclear power industry with a performance-based rule to improve the 
overall effectiveness of maintenance programs and integrate risk considerations into maintenance processes. Application of this rule and good maintenance practices should provide for detection and accommodation of any degradation of active SSC performance. The Rule requires that each NPP operator set goals to monitor the SSCs in order to ensure that those SSCs are capable of performing their intended function, even in design basis accident scenarios. Representative measurands are used to quantify, monitor, and trend performance to detect equipment performance degradation and aging. These measurands may include flow, pressure, vibration, or temperature for monitoring individual components. Failures that are likely to cause loss of an intended function should be monitored through these unitspecific measurands to give early warning of degradation. However, when this is not the case, reliability and availability are traditional measurands for monitoring the overall health of systems. Risk-significant systems must be monitored at the individual, or train, level to ensure that poorly performing systems are not being compensated for by higher-performing redundant systems. Additionally, common-cause failures and generic problems are monitored across similar components in different system component groups. A review of the Rule is given by Gregor and Chockie (2006) and Stevenson (2006).

The Rule was issued in 1991 and became fully effective in July 1996. Guidance for applying and fulfilling the Rule is given in NUMARC 93-01 (1996), which was endorsed by NRC (1997). The scope of the rule, defined in section (b), includes (1) safety-related SSCs that are relied upon to remain function during and after design basis accidents and (2) nonsafety-related SSCs that are relied upon to mitigate accidents or transients, that could prevent safety-related SSCs from fulfilling their functions, or that could cause a reactor scram or actuation of a safety-related system through their failure. Most NPPs use an expert panel to determine which SSCs fall into each of these categories. The NRC reviews the application of the Rule and NUMARC guidance at each NPP in the United States. As utilities began to develop maintenance monitoring programs to fulfill the Rule leading up to 1996, lessons of early efforts at implementation were summarized in Petrone et al. (1995). These early inspections found that utilities were largely applying the Rule accurately to develop maintenance monitoring programs. After the Rule had been in place for a few years, another review of baseline inspections was performed which found that the Rule was well-understood and appropriately applied for monitoring maintenance effectiveness (Wong et al. 1999). While the Rule has been adequate for monitoring the effectiveness of maintenance of active components, it does not directly improve the scheduling or economics of performing maintenance. In fact, most maintenance activities remain periodically scheduled under the Rule; however, well-applied condition-based maintenance (CBM) could reduce unnecessary maintenance and cost, and its effectiveness could also be monitored under the Rule.

\subsection{Passive Components - Aging Management Plans}

Management of aging and degradation of passive components and structures is accomplished through the plant's aging management plan (AMP) and scheduled in-service inspection (ISI). The AMP applies to all SSCs that are safety-related or whose failure could affect safety-related functions, as well as those SSCs relied on for compliance with fire protection, environmental qualification, pressurized thermal shock, anticipated transients without scram, or station blackout regulations. The NRC's guidance with regard to AMPs is provided in a set of reports referred to as the Generic Aging Lessons Learned (GALL) reports (NRC 2001, 2005a, b, 2010d). These reports provide the technical basis for determining whether aging management programs at operating reactors are adequate or need modification as plants enter extended operation. Specific programs that need modification are also identified, and the information in 
these reports are included in the NRC's Standard Review Plan for Review of License Renewal Applications (NRC 2010c).

A key component of the AMP is the scheduled ISI of passive components, codified in 10 CFR 50.55a (2007), which specify the requirements for nondestructive inspection (such as inspection periodicity, inspection techniques, and qualification procedures). These elements are contained in the American Society for Mechanical Engineers (ASME) Boiler \& Pressure Vessel (BPV) Code, which the Code of Federal Regulations incorporates by reference. The ASME Code specifies the minimum requirements for nondestructive examination (NDE). Specifically, Section XI of the Code defines the acceptable volumetric and surface examination techniques, minimum requirements for acceptable procedures, and the acceptance criteria for flaws that are detected. In addition, requirements for qualification of the procedures, equipment, and personnel are specified to ensure reliable inspections.

Reliability of the nondestructive ISI techniques is key to ensuring that the defense-in-depth philosophy works as intended. In this context, reliability includes several factors, such as the minimum detectable flaw, critical flaw size, and the probability of detection (POD), which is a function of equipment, inspection method that is implemented (procedure), and human factors (such as operator training, inspection environment, etc.) (Mueller et al. 2012). The reliability of NDE was originally examined in several studies conducted by the US Air Force (Singh 2000). Concerns regarding the ability of available NDE techniques to detect critical flaws in safety-related components in nuclear power plants caused the NRC and other organizations (in the United States and overseas) to conduct a series of studies on the reliability of ultrasonic NDE techniques (Chockie 1981; Doctor 1984; Fong 1986; Bates et al. 1987; Nichols and McDonald 1987; Willetts and Ammirato 1987; Doctor et al. 1995; Doctor 2007; Miller 2008). These studies demonstrated that several sources of variability were present that impacted the reliability of NDE, and that an appropriate performance demonstration procedure was needed to ensure consistent reliability in field examinations. These results were codified into the ASME BPV Code, in Section IX, Appendix VIII, and are also the basis for performance demonstration procedures for other NDE techniques (Chockie 1985).

In the United States, for certain inspection techniques and components, the nuclear industry has developed additional examination guidelines, such as those developed under the Boiling Water Reactor Owners Group's Vessel and Internals Project (BWRVIP) program, and the Materials Reliability Program (MRP) (for instance, EPRI 2008c; EPRI 2011c). In addition, the concept of risk-informed inspections is being explored (IAEA 2010), which categorizes SSCs as being of either high or low safety significance. The AMP is then tailored to ensure that high-safety significance SSCs are inspected, while maintaining some minimum inspection coverage of low-safety significance SSCs.

One persistent issue with the operating plants is that these were generally designed without consideration for in-service requirements; indeed, the ISI and AMP programs were established only after these plants were built and operating (Shah and MacDonald 1993; Tipping 2010). The result is that several parts of the plant structures and components are not amenable to easy inspection, and the NRC therefore annually has to handle several requests for relief from the ISI requirements for these components. Some of these issues are being addressed in the licensing for next-generation reactors, with particular attention being paid to inspectability (EPRI 2007). Such changes, in concert with new and advanced online nondestructive monitoring methods such as acoustic emission, are expected to form the technical basis for safe extended operation in the future. 



\subsection{Technical and Economic Motivations}

This section discusses the technical and economic challenges that make PHM attractive for both legacy plants and new plant designs. While the traditional approaches to maintenance and degradation management (the Maintenance Rule and Aging Management Plan) have been adequate in the past, these approaches are generally not optimized in terms of effort, time, or cost. As plants move into longer service lives (both through LWRS and extended license periods of newer designs), the frequency of periodic inspection and maintenance may have to change to compensate for potentially increasing failure rates of known fault modes and heretofore unknown fault modes. Advanced reactor designs introduce an additional set of challenges with exposure to potentially more severe stressors and the use of new, innovative materials to accommodate them. Condition monitoring and PHM may have the potential to improve plant safety, increase efficiency, and reduce operating costs in NPPs, both legacy and future.

\subsection{Light-Water Reactor Sustainability}

Of the 104 NPPs in operation in the United States, 71 have received the first round of license extension, from 40 to 60 years of operation, and most of the remaining plants' applications are under review. To date, nine U.S. plants have entered extended operation. Industry polls indicate that license renewal is largely supported by the public, with $81 \%$ of respondents in favor of extending operation of existing plants (Bisconti 2007). A review of the practical issues of policy and environment for license renewal concludes that advanced monitoring technology is key for extending licenses (Cerafici 2009). As of December 2011, nine plants have entered long-term operation (LTO); that is, operation beyond the original 40-year licensing period (NRC 2011). Consideration is already being given to the second round of license extensions for operation from 60 to 80 years, called extended LTO. Light Water Reactor Sustainability (LWRS) refers to the research and development programs designed to support safe and economic operation of the existing reactor fleet during LTO and extended LTO. To continue to provide secure, reliable nuclear power generation, it is imperative to understand and manage the challenges posed by NPP system aging.

Aging is a potential problem for both active components (which exhibit increasing failure rate over time due to wear-out failures) and passive structures (whose safety margins are being reduced toward the lowest allowable level). Historically, active components have been well maintained, managed, diagnosed, repaired, and replaced as necessary under the Maintenance Rule. However, the application of advanced prognostics and health monitoring methods may support the economics of LWRS as the maintenance of these components shifts from conservative, periodic maintenance scheduling to just in time repair. The health assessment of passive structures and components will necessarily need to transition to a more continuous approach to ensure their intended functions will be maintained for the period of operation and extended operation. Additionally, advanced monitoring of passive structures may provide warning of incipient fault and failure in advance of traditional ISI techniques, further ensuring that intended functions are maintained over the same period.

Redundancy is one of the principal barriers against the negative effects of random failures; however, as systems age, the likelihood of simultaneous failures of redundant safety systems becomes more compelling. Concerns over the effects of aging on plant SSCs have been building for at least the past 25 years (Novak and Podest 1987). Currently, there is increased interest in using condition-based rather than time-based maintenance for active components and automated online monitoring instead of periodic 
ISI for passive structures to support LWRS (Jarrell et al. 2004; Bond et al. 2008c; Bond et al. 2008d; Bond and Meyer 2011).

\subsection{Generation III+, IV, and Future Plants}

The U.S. fleet of operating LWR plants is largely considered Generation II (Gen II) designs. Generation III and III+ reactors are advanced LWR designs. Gen III reactor designs improve on Gen II by incorporating improvements that have developed during the operation of Gen II reactors. Gen III designs commonly include improved fuel technology, thermal efficiency, passive safety systems, and a standardized design for multiple units. Worldwide, several Gen III plants have been built and operated, including four advanced boiling water reactors (ABWR) in Japan. Gen III+ designs, such as the AP-1000, the European Pressurized Reactor (EPR), and the Economic Simplified Boiling Water Reactor (ESBWR), offer further improvements in safety and economics over the Gen III designs. Several Gen III+ plants are currently under construction, including EPR plants in Finland, France, and China. On-line monitoring (OLM) is now being deployed as part of new LWR plants; for example, by AREVA in the new EPR reactor at Olkiluoto in Finland, where 256 online measurement channels are included (AREVA 2007).

Development and deployment of small-scale nuclear power reactors (generally considered less than $350 \mathrm{MW}$ ) and their maintenance, monitoring, and control are part of the mission under the U.S. DOE Small Modular Reactor (SMR) program. Ingersoll (2009) describes SMRs as "deliberately small reactors" with modular characteristics and multiple units deployed at the same plant site. SMRs are designed to potentially operate in remote locations with limited infrastructure and skilled personnel. These reactors have additional monitoring concerns over traditional nuclear plant designs; these needs include increased expectations of availability, longer operating cycles between planned refueling and maintenance opportunities, and increased concerns for safety and proliferation resistance.

Both LWR-based and advanced reactor designs for SMRs will necessarily have significantly different I\&C needs than the existing reactor fleet (Clayton and Wood 2011). LWR-based designs may include integral primary systems, which will have sensor access requirements very different from existing LWRs. The location of active components, such as pumps, inside the reactor vessel limits the ability to perform periodic diagnostic measurements; automated, online measurements for these components will be necessary to provide surveillance and health assessment. Additionally, unconventional components, such as helical coil steam generators, do not have well-established performance and degradation histories. Continuous surveillance of these systems will alleviate some of the uncertainty associated with deploying unproven technology. Advanced SMR designs commonly use different coolants and operate at higher temperatures than traditional LWRs, which may pose problems for existing sensor technology. There are significant challenges associated with the choice of materials that are compatible with the unique operating conditions expected for advanced designs. New forms of degradation (such as hightemperature creep, oxidation/carburization, etc.) in passive components such as heat exchanger tubing, pressure boundary components, and reactor internals are anticipated due to these operating conditions. In addition, there are a host of advanced structural materials being proposed for use in SMRs (such as ODS steels) where the long-term structural performance is not well understood. Thus, the development of rapidly growing degradation in passive components is likely to impact the safe long-term operation of SMRs if not detected in a timely manner. Traditional approaches based on periodic in-service nondestructive inspection methods (such as those used in current LWRs) for detecting such degradation 
are not applicable to SMRs, given the expectation of longer operating periods between re-fueling outages, and potential lack of inspection access to critical components. Many of these designs are intended for energy applications in addition to electricity production, such as process heat production. Longer operating cycles will also affect the online monitoring and surveillance needs of SMRs. Most LWRbased designs anticipate a 3- to 4-year operating cycle, while some advanced concepts project core lifetimes of 20- to 30-years. In both cases, outage frequency may be driven by inspection and maintenance needs, which makes timely, accurate detection of faults and incipient failures even more paramount. Proponents of SMR designs also anticipate reduced maintenance staff as a cost-saving feature. While the maintenance requirements for an individual reactor may be reduced, the total workload at a specific site will increase with the number of plants (and, therefore, the number of systems and components) located at the site. Again, accurate, timely detection of faults and projection of RUL will help alleviate the maintenance burden by focusing efforts on those SSCs which need maintenance.

Several advanced concepts have been proposed as part of Generation IV initiative to develop the future fleet of nuclear power generating plants. Most of the concepts represent significant departures from current LWR technology inspired by efforts to improve existing technology in one or more areas including increased electricity generation efficiency, minimization of waste, implementation of passive safety features, and improved proliferation resistance. A review by Abram and Ion (2008) identifies six Generation IV concepts, including the very high-temperature gas-cooled reactor (VHTR), the gas-cooled fast reactor (GFR), the sodium-cooled fast reactor (SFR), the lead-cooled fast reactor (LFR), the molten salt reactor (MSR), and the super-critical water-cooled reactor (SCWR). Robinson (2006) presents a general framework for processing monitoring data and maintenance records to characterize current and future performance and to estimate current and future failure probabilities for subsystems and components of advanced reactors. A key challenge in developing PHM systems for future systems is the lack of operating data to train and validate PHM models. An adaptive approach has been proposed to move from high-fidelity simulations developed in the design phase to actual operating data as it becomes available (Hines et al. 2011).

Several experimental and prototype reactors have been built that demonstrate VHTR and SFR technologies while demonstrations of LFR and MSR technologies have been more limited. GFR or SCWR reactor technologies have yet to be demonstrated (Abram and Ion 2008). In contrast, global experience in the operation of LWRs is extensive, yet knowledge of materials degradation behavior in LWRs is incomplete, particularly when considering LTO and extended LTO. Advanced reactors will pose a considerably greater challenge, given that thermal, chemical, and radiological stressors will be more extreme. To meet this challenge, many advanced reactor components will likely be fabricated from new or relatively unfamiliar materials. As a consequence, "surprises" must be anticipated with respect to materials aging in advanced reactors; a proactive approach to aging management incorporating online monitoring, measurements of early degradation, and prognostics can help alleviate the burden of operating new reactor designs. In addition, certain advanced reactor designs, such as the Pebble Bed Modular Reactor (PBMR), are designed to refuel online, reducing the frequency of convenient opportunities for maintenance. Implementation of proactive aging management concepts can help operators of these reactors cope with extended periods between maintenance. 


\subsection{Business Case for PHM}

The current U.S. nuclear power fleet represents about $10 \%$ of installed capacity and it is used to generate approximately $20 \%$ of U.S. electricity. This base load generation remains the most economical source of electricity in the United States (Nicholson et al. 2011; WNA 2011). Operations and maintenance (O\&M) costs comprise approximately $60-70 \%$ of the overall generating cost in NPPs, while only $15-30 \%$ of costs are attributed to fuel. Furthermore, of the O\&M costs in U.S. plants, approximately $80 \%$ are labor costs (Wacker et al. 2007). The use of PHM has potential to impact the economics of maintenance for both active and passive SSCs (Bond et al. 2008b; Bond et al. 2012 (draft)).

The United States faces an additional challenge due to the coal-fired power plant fleet, which is also aging. On December 21, 2011, the Environmental Protection Agency (EPA) issued the Mercury and Air Toxics Standards, which require the reduction of energy-related emissions, largely from coal-fired power plants, over the next three years. Because the cost to employ these pollution-control measures will be necessarily passed on to consumers, nuclear-generated power is likely to remain an economically competitive form of electricity generation.

At the time of this writing (2012), natural gas is being considered an economic alternative to nuclear power (Barron 2012). Primarily, this is based on the present low cost of natural gas. However, recent studies show that the total cost of gas-fired plants are a function of several other factors, including market regulation, transportation, building and maintaining the plant, electric grid upgrades, and any escalation in the supply cost of natural gas and the cost of electricity (Barron 2012). As a result, nuclear generation may remain an economically competitive alternative to natural gas.

Equipment maintenance has some associated fixed cost for labor, repair and replacement parts, and the minimum necessary downtime for repair. Unscheduled maintenance due to unexpected equipment degradation or failure can incur significant additional costs, including possible secondary degradation or failure that may result from an in-service equipment failure. The time needed to perform unscheduled maintenance may be extended due to a lack of necessary parts, equipment, and crew and the added repair time for secondary failures. The lost revenue from reduced or halted electricity production during this extended repair time is a significant cost, estimated at approximately $\$ 1.25$ million per day of plant shutdown for an average plant in the United States (NEI 2011). Discovery of unanticipated pressure vessel head degradation at the Davis-Besse nuclear plant led to a 25-month outage and estimated repair costs exceeding $\$ 600$ million. In September 2008, a turbine generator malfunction at the D.C. Cook nuclear plant resulted in a fire, which led to eventual manual plant shutdown. Turbine repairs totaled \$332 million in addition to lost revenue during the one-year outage. Two separate instances of cooling tower collapse in August 2007 and July 2008 at the Vermont Yankee plant led to a reduction in power generation to approximately $35 \%$ capacity for 11 and 12 days, respectively. Obviously, it is of paramount importance to be aware of impending SSC failures so that preventive maintenance can be performed, operations can be adjusted, or auxiliary equipment can be employed to avoid these costs when possible. Information from online monitoring and prognostics enables turning unscheduled maintenance actions (resulting from unexpected malfunction of SSCs) into scheduled work.

The results of the Nuclear Plant Aging Research (NPAR) program and complimentary industrydriven aging research programs indicate that the aging phenomena in existing NPPs do not pose an insurmountable technical challenge to life extension; the life of these plants is primarily limited by the cost of effective inspection and maintenance programs and the cost of repair or replacement of degraded 
SSCs. Legacy commercial aircraft face many of the same challenges that legacy NPPs face in continued operation, including both policy challenges (i.e., significant regulations that control technology certification and application) and technical challenges (i.e., lack of data collection and processing infrastructure in legacy systems). From a business perspective, the goal in applying PHM to the commercial aircraft industry is identical to that of the legacy NPP fleet: to yield maximum profit while maintaining safe operation. Leão et al. (2008a) present a cost-benefit analysis (CBA) for applying PHM to the aging commercial aircraft fleet to extend life and alleviate unnecessary maintenance. Many of the enumerated benefits and costs are common to both industries. Benefits include increased system availability and reliability; reduction in scheduled maintenance tasks and associated costs; reduction in secondary damage due to failure of a primary component; reduction in maintenance-induced failures (due to unintentional damage caused during normal maintenance activities); reduction of insurance costs; and intangible benefits, including building a positive public perception of maintenance and safety practices. Common sources of cost include PHM system development and certification; deployment of additional sensors and processing capabilities; maintenance of PHM system, including replacing and repairing sensors and maintaining PHM models; training of appropriate personnel; and additional maintenance performed due to inaccurate or conservative prognostic models. Quantification of the individual figures prescribed to each benefit and cost is difficult due to changes over time and the uncertainty in equipment condition that makes prognostics necessary. Cost of new technology deployment and related issues, such as replacement of instrumentation cabling, in a given plant may be the economic decider for the feasibility of extended operation (Bond et al. 2011b).

Anecdotally, appropriate application of PHM reduces the cost of maintenance while increasing system availability and reliability. No formalized CBA for applying PHM in a specific NPP has been found; however, analyses by Bond et al. (2011b) and Wacker et al. (2007) suggests that fleet-wide savings of over $\$ 1$ billion per year are possible in the United States when PHM is applied to all key equipment in legacy power plants. Analyses of PHM in other industries and in general suggest significant potential savings. Duc and Ming (2011) compare the costs of different maintenance policies, including two scheduled maintenance plans and two CBM plans. The CBM systems do not include the use of prognostics to schedule maintenance; maintenance is performed when a sensed indicator exceeds a predefined threshold. Even with these naïve maintenance policies and under varying costs of failure and maintenance quality, the two CBM methodologies consistently present significant cost savings over the scheduled maintenance plans. Banks and Merenich (2007) apply a trade space visualization software to CBA, which accounts for the cost associated with operational unavailability, a significant cost for NPPs. Hecht (2006) investigated application of PHM to electronic equipment and identified several general results that may be widely applicable, including the use of broad spectrum sensors to cover a large number of failure mechanisms with minimal hardware; targeted application of PHM sensors and algorithms to those where the difference in cost between pre-planned and unanticipated maintenance is high; and managing the cost of sensors and implementation to ensure some savings are realized if prognostic coverage is not as high as expected. Tian et al. (2011a; 2011b) give a methodology to economically optimize maintenance planning of multi-component systems based on condition monitoring results. This method capitalizes on fixed costs associated with any maintenance action, such as sending a maintenance team to the site, by combining many maintenance actions that will be necessary in some short-term window into one maintenance event. Drummond and Yang (2008) present a different approach to CBA by determining a range of key features (cost of failure, failure rate, false alarm cost, etc.) over which the algorithm is economically useful compared to schedule-based and run-to-failure maintenance policies. The existing literature on CBA for PHM applied to a variety of components and 
systems suggest a strong business case for applying PHM to maintenance planning and life extension of SCCs in legacy NPPs; however, this analysis will need to be performed on a plant-by-plant basis. The case for PHM in future NPPs is even stronger, because the added cost of expensive retrofitting can be avoided by incorporating sensors, communication infrastructure, and other PHM considerations beginning in the design phase.

Considering the lessons learned in operating and maintaining the existing fleet of NPPs, new reactors will clearly benefit from including advanced monitoring, fault detection, diagnostic, and prognostic infrastructure from initial design through operation. Retrofitting health monitoring systems to existing plants is more costly and likely more complicated than incorporating the necessary monitoring systems in the design phase. However, in both legacy and future plants, the economics of PHM for NPPs is attractive. 


\subsection{Introduction to Prognostics and Health Management Systems}

Several related programs have been proposed since the late 1990s to facilitate the move from findand-fix maintenance policies to predictive maintenance. The Joint Strike Fighter (JSF) research group includes fault detection and isolation, enhanced diagnostics, material condition assessment, performance monitoring, and estimation of remaining useful life (RUL) under the umbrella of prognostics (Hess et al. 2005). However, this collection of activities seems better suited to the common moniker of Prognostics and Health Management (PHM) or the program suggested by the U.S. Deputy Under Secretary of Defense for Logistics and Material Readiness, Condition Based Maintenance plus (CBM+) (Jaw and Merrill 2008; Millar 2009). Full health monitoring systems, also called Condition Based Maintenance (CBM) systems, are the focus of much research. Callan et al. (2006) outline a five-step CBM system, which includes: data acquisition, data manipulation, condition monitoring, health assessment, and prognostics. Walter (2006) extends this system with a sixth module: advisory generation. Pipe (2008) and Hess et al. (2005) suggest the use of RUL estimates for maintenance planning and logistics systems. Kothamasu et al. (2006) describe using prognostic estimates to aid maintenance scheduling and planning; they also suggest prognostics for optimal control algorithms.

The Life Extension Analysis and Prognostics (LEAP) program focused on health monitoring of complex mechanical systems for diagnostics, prognostics, and maintenance scheduling (Greitzer et al. 1999b). One outcome of the LEAP research program was a prognostics architecture concept that helped to communicate logistics and organizational requirements fundamental to establishing capabilities for anticipatory logistics that exploit prognostics analyses for different applications and multiple analytical methods (Greitzer 2000). This has led to a six-step PHM development process (Greitzer and Ferryman 2001):

1. Conduct a thorough failure modes and effects analysis (FMEA) to identify maintenance issues and high-frequency/high-cost faults and failures;

2. Identify sensors that are necessary to diagnose and/or predict faults;

3. Conduct a cost-benefit analysis to determine the monetary and intangible impacts;

4. Design and fabricate additional sensors, if necessary;

5. Collect testbed and field data for system development and validation; and

6. Develop and apply prognostic algorithms in a prototype version of the PHM system.

The LEAP program also resulted in a prognostic methodology termed LEAP-Frog, which attempts to trade-off between short- and long-window regressions to provide optimal response to changing system conditions while controlling prognostic uncertainty (Greitzer 2001a).

Figure 4.1 illustrates the modules of a typical health monitoring system. By applying the entire suite of modules, one can accomplish the goals of most prognostic systems: increased productivity; reduced downtime; reduced number and severity of failures, particularly unanticipated failures; optimized operating performance; extended operating periods between maintenance; reduced unnecessary planned maintenance; and reduced life-cycle cost. Data collected from a system of interest is monitored for deviations from normal behavior. Monitoring can be accomplished through a variety of methods, 
including first-principle models, empirical models, and statistical analysis (Hines and Seibert 2006). If a fault is detected, it is often important to identify the type of fault; systems will likely degrade in different ways depending on the type of fault and so different prognostic models will be applicable. Expert systems, such as fuzzy rule-based systems, are common fault diagnosers. With this information, a prognostic model is employed to estimate the RUL of the system. This model may include information from the original data, the monitoring system residuals, and the results of the fault detection and isolation routines. Finally, knowledge of the estimated current and predicted future system health can be used to inform O\&M planning or for optimal, fault-accommodating/fault-adaptive control. Table 4.1 assesses the maturity of diagnostics and prognostics for several classes of SSCs and application spaces.

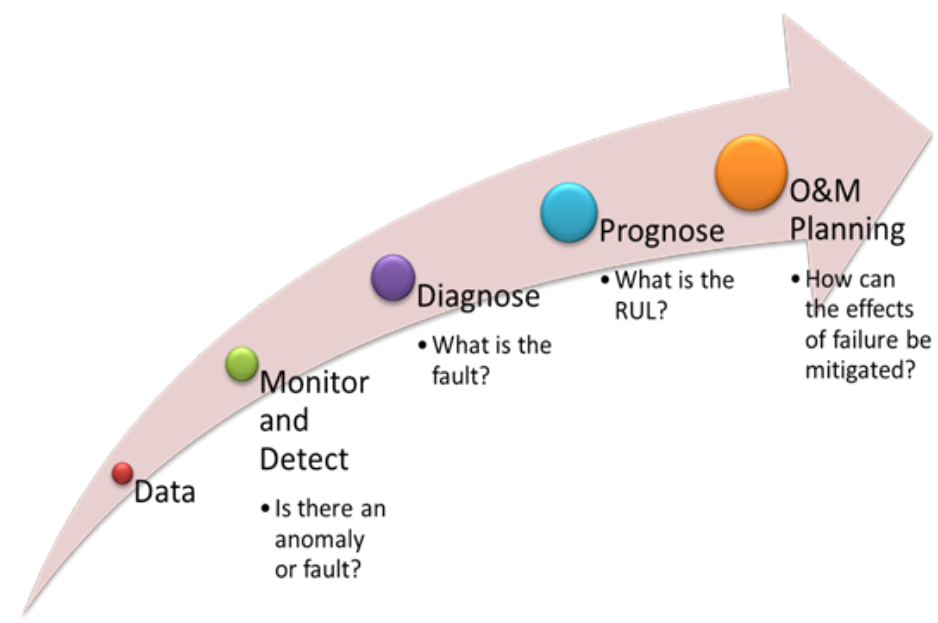

Figure 4.1. Suite of Modules in a Health Monitoring System (Hines et al. 2008b)

Table 4.1. Assessment of State of Maturity for Diagnostic [D] and Prognostic [P] Technologies (adapted from (Howard 2005; Bond et al. 2008a)

\begin{tabular}{|c|c|c|c|c|}
\hline Diagnostic/Prognostic Technology for: & $\mathbf{A P} \mathbf{P}^{(\mathbf{a})}$ & $\mathbf{A}^{(\mathbf{b})}$ & $I^{(\mathbf{c})}$ & $\mathbf{N O}^{(\mathrm{d})}$ \\
\hline Basic Machinery (motors, pumps, generators, etc.) & $\bar{D} \& \mathrm{P}$ & & & \\
\hline Complex Machinery (helicopter gearboxes, etc.) & $\mathrm{D} \& \mathrm{P}$ & & & \\
\hline Metal Structures & $\mathrm{D}$ & $\mathrm{P}$ & & \\
\hline Composite Structures & & $\mathrm{D}$ & $\mathrm{P}$ & \\
\hline Electronic Power Supplies (low power) & $\mathrm{D}$ & $\mathrm{P}$ & & \\
\hline Avionics and Controls Electronics & $\mathrm{D}$ & $\mathrm{P}$ & & \\
\hline Medium Power Electronics (radar, etc.) & $\mathrm{D}$ & $\mathrm{P}$ & & \\
\hline High Power Electronics (electric propulsion, etc.) & $\mathrm{D}$ & $\mathrm{P}$ & & \\
\hline Instrument Re-calibration - monitoring (NPP) & $\mathrm{D}$ & & & $\mathrm{P}$ \\
\hline Active Components - nuclear power plants & $\mathrm{D}$ & & $\mathrm{P}$ & \\
\hline Passive Components - nuclear power plants & & & $\mathrm{D}$ & $\mathrm{P}$ \\
\hline $\begin{array}{l}\text { (a) } \mathrm{AP}=\text { Technology currently available and proven effective. } \\
\text { (b) } \mathrm{A}=\text { Technology currently available, but } \mathrm{V} \& \mathrm{~V} \text { not completed. } \\
\text { (c) } \mathrm{I}=\mathrm{Tech} \text {. } \\
\text { (d) } \mathrm{NO}=\text { No significant technology development in place. }\end{array}$ & & & & \\
\hline
\end{tabular}


Each module in the PHM system is described in the sections below, from data collection through prognostics. Incorporating this information into control and O\&M planning is an area of active research and is described later in this report.

\subsection{Data Collection - NDE for Passives, Variable Sensing for Actives}

Traditional reliability-type analysis estimates the probability of faults and failures in a system based only on population distributions, but this does not give any insight into the current or future condition of a specific SSC. Information from an SSC of interest is necessary to make an accurate, reliable prediction of individual health.

Active components in NPPs include pumps, valves, motors, sensors, etc. For these active components, PHM systems can capitalize on the information already collected by the plant I\&C system: temperature, flow, pressure, etc. Pump health may be estimated using discharge pressure and flow; valve operation could be monitored through the changes in flow as the valve position setpoint is changed; and sensor calibration can be monitored and diagnosed by using the data those sensors are collecting.

For some active components, additional measurements may be useful or necessary to develop more robust and accurate prognostic models. Pumps and motors can be monitored through vibration measurements; in fact, reactor coolant pumps and casing are commonly monitored through the reactor coolant pump vibration monitoring system (RCPVMS) (Koo and Kim 2000). However, these systems do not currently support automated, online analysis of the vibration data to detect and diagnose abnormal conditions. Motors, such as those used for motor-operated valves, can be monitored through multiple features, such as input current and voltage, active power, motor position measures, and applied forces. These monitoring methods using additional data sources are described in more detail in Section 6.1. Many of these additional measurements, such as vibration, motor position, or electrical signatures, may largely be obtained autonomously, online, and unobtrusively.

Similar measurement capabilities are not as readily available for passive components. Damage in passive components takes many forms (corrosion, cracking, etc.) and typically results in a localized change in material properties (electrical or thermal conductivity, magnetic permeability, elastic modulus, etc. (Raj et al. 2003)). Measurements that are sensitive to these discontinuities are generally used to detect damage in passive components in a nondestructive manner. Nondestructive measurement methods available for passive components include radiography, ultrasonic imaging, visual inspection, electromagnetic inspection (including eddy currents, potential drop methods, etc.), and thermal imaging (Meyer et al. 2010; Meyer et al. 2011a). Almost all of these methods (with the exception of visual inspection) are focused on the detection of hidden damage in components, and rely on the interaction of applied energy with the material damage sites. In most cases, the inspection process requires progressive scanning to inspect the entire component, and may require significant intervention (i.e., not autonomous, online, or unobtrusive). As with active components, the resulting data only provides a snapshot of the current condition of the system.

Exceptions (for passive component nondestructive condition measurements) include wide-area inspection methods such as acoustic emission (AE) monitoring and guided ultrasonic waves (GW). AE relies on the (usually long-range) detection of stress waves that emanate from damage sites, either intrinsically due to the growth process of the damage or as a result of an external applied load to the 
component. AE is well suited for online monitoring of passive components in nuclear power plants, and has been applied for crack growth detection (Harris and Dunegan 1974; Hutton et al. 1984; Hutton 1993; Hutton et al. 1993; Ai et al. 2010; Meyer et al. 2011b), leak detection (IAEA 2008b), and loose part monitoring (IAEA 2008b). GW also relies on the interaction of long-range stress waves with damage in the component (Rose 1999; Meyer et al. 2012b). However, GW is an active technique that uses transducers to generate guided waves in the structure, and records the result of the interaction. The guided waves that are used in GW inspection are able to propagate over long distances (10s of meters) enabling wide-area inspection (Rose 2002). A brief review of applications of GW for nuclear power plant components is provided in (Meyer et al. 2012b).

The methods described above are focused on detecting the presence of damage in materials that result in a change in the local material properties. Inspection methods that rely on modal frequency analysis for structural monitoring have been proposed in other application spaces (Vipperman 1999; Zimmerman et al. 2008). These methods typically use accelerometers to measure displacements at different points in the components and use the result to analyze changes in primary modes of vibration of the structure. Changes in the modal structure are usually correlated to the level of structural damage.

\subsection{Monitoring Methods}

Condition monitoring describes a suite of activities for estimating system state and providing early warning of anomalous behavior. Requirements for extending data collection to equipment condition assessment include (Rasmussen 2005):

- clear understanding of all failure modes of interest,

- installed instrumentation capable of detecting these failure modes,

- defined instrument signatures for each failure mode,

- significantly slow progression from fault to failure to provide early detection,

- unambiguous early detection to provide actionable information, and

- negligible probability of false- and missed-alarms.

Detailed reviews of state estimation and fault detection methods are given in Hashemian (1995), Hines and Seibert (2006), Heo (2008), and Ramachandran et al. (2010). State estimation and fault detection will be briefly described in the following sections. State estimation methods can be roughly divided into physical models and empirical models.

\subsubsection{Physical Models}

If the underlying physical mechanisms of a system are well understood, then an analytical model based on first principles can be designed to describe the expected nominal (or in some cases faulted) behavior based on measured system features or operating conditions. For instance, if the pressure of saturated steam at the outlet of a steam generator in a PWR is measured, the temperature can be determined based on well-known physical relationships. Physical models are attractive for engineering systems because they explicitly account for mechanical, material, and operational characteristics; they can be developed and evaluated before the system has been built and operated; and they can be applied to a 
wide variety of operational and material conditions to understand behavior over a wider range of operation. However, these models can be costly and time-consuming to develop for large, complex systems, and developed models often have limited applicability. Additionally, simplifying assumptions are often necessary for phenomena that are not fully understood or to improve runtime performance.

\subsubsection{Empirical Models}

Unlike physical models, empirical models are built on historical operation data with no explicitly defined understanding of the underlying physical mechanisms of the system. These modeling methods can be classified according to two characteristics: parametric versus nonparametric and inferential versus auto-associative (Table 4.2). Parametric models use the available data to determine the parameter values for a functional fit and then "throw away" the historic data; a well-known classic parametric model is linear regression. Conversely, nonparametric models retain the historic exemplars in a memory matrix and include an algorithm for combining these exemplars to make a prediction for each new observation (e.g., kernel regression). Inferential models use a set of explanatory variables to predict one response variable; again, a classic example is linear regression. Autoassociative models predict the "correct" set of variable measurements given the measured values of those variables; here, the inputs and the outputs represent the same variables. These state estimation methods can be thought of as error correction routines.

Table 4.2. Categorization of Select Empirical State Estimation Methods

\begin{tabular}{ccc}
\hline & Parametric & Nonparametric \\
\hline & Linear Regression & Kernel Regression \\
& Neural Networks & Locally Weighted Regression \\
& & \\
\hline
\end{tabular}

All of these state estimation techniques, both physical and empirical, inherently have uncertainty associated with their outputs. Sources of this uncertainty include instrument channel uncertainty (electrical and sensor noise, instrumentation faults, process variation) and estimation model uncertainty (integrity and appropriateness of the model). Statistical monitoring techniques can be used to determine if system operation is nominal or faulted while taking these uncertainties into consideration; the next section describes some common fault detection routines.

\subsubsection{Fault Detection Methods}

The simplest method of fault detection is thresholding. Thresholding monitors a sensed value (or state estimation residual) and alarms when it exceeds some predefined, fixed threshold. This type of fault 
detection looks for gross changes in the sensor value. Care must be taken when setting the alarm threshold to balance between false alarms (due to noise naturally present in the system) and missed alarms (common when faults only induce small changes in a single variable). Advanced fault detection methods typically compare the nominal system state estimated by a physical or empirical model to the system state measured by sensed variables to detect discrepancies between expected and actual behavior. The difference between expected and actual behavior, called the residual, characterizes system deviations from normal behavior and can be used to determine if the system is operating in an abnormal state. Statistical methods are commonly used in this analysis to account for noise and uncertainties in the predicted and measured system states. Several reviews of fault detection routines are available (Isermann 1984; Gertler 1988; Angeli and Chatzinikolaou 2004; Miljkovic 2011). Three common routines are described here: error uncertainty limit monitoring, sequential probability ratio test, and control charts.

Error Uncertainty Limit Monitoring (EULM) fault detection is an adaptation of simple threshold monitoring developed specifically for sensor calibration monitoring in NPPs. EULM fault detection adapts simple thresholding for use in the nuclear power industry by monitoring the uncertainty bounds about a sensed value (or state estimation residual) and alarming when the uncertainty bounds exceed some threshold, as shown in Figure 4.2. The calculation of drift limits inherently accounts for the imperfect estimation of monitoring system residuals by incorporating the effects of faulty inputs on modeling outputs through the model's auto-sensitivity. This approach offers an additional level of conservatism to the fault detection, indicating when the monitored parameter is no longer within the error thresholds to some specified confidence level.

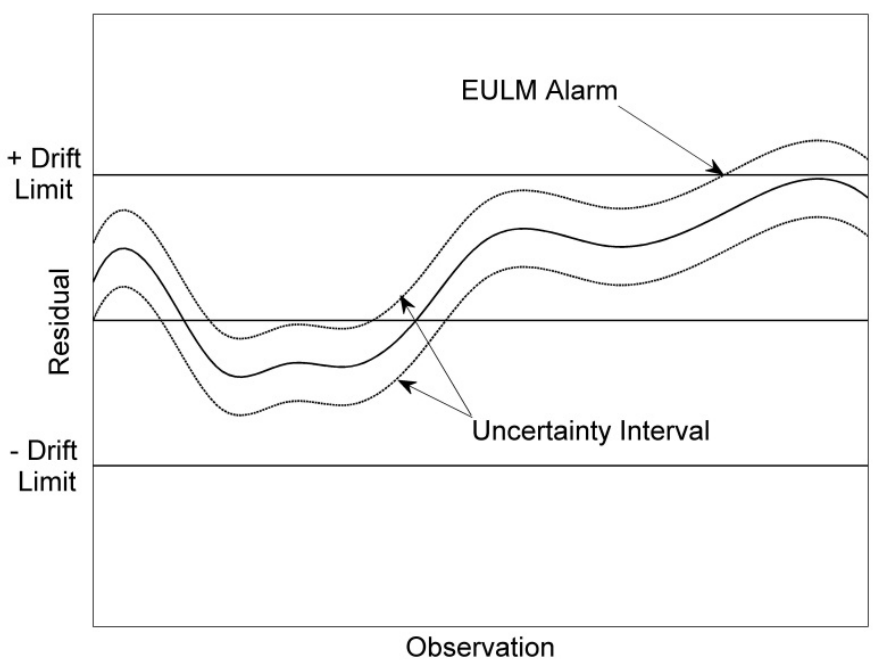

Figure 4.2. EULM Fault Detection

The sequential probability ratio test (SPRT) was developed by Wald (1945). This statistical test considers a sequence of residuals and determines if they are more likely from the distribution that represents normal behavior or that of a faulted distribution, which may have a shifted mean value or altered standard deviation from the nominal distribution. Figure 4.3 shows the nominal and faulted distributions for a SPRT test for a positive shift in mean value. Here, the faulted distribution must be predefined to perform the sequential analysis. If the behavior of expected faults is not well understood, the 
faulted distribution can be defined to achieve specified false- and missed-alarm probabilities. However, this method is based on the assumption that noise and residuals are normally distributed and white, which may not occur in practice.

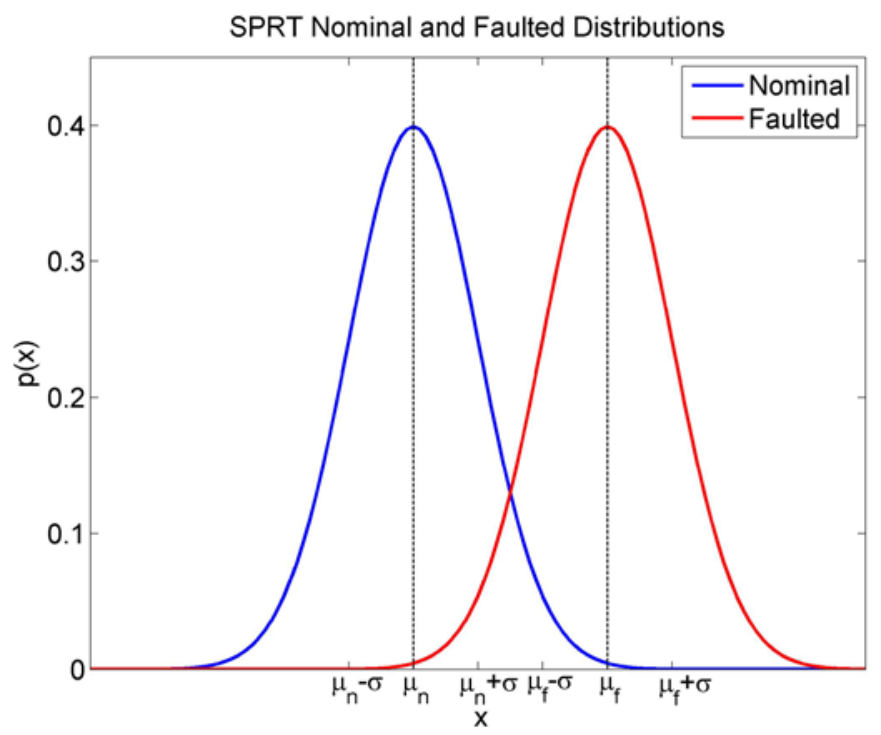

Figure 4.3. Nominal and Faulted Gaussian Distributions

A control chart, also called a Shewhart Chart, indicates if an engineering system is statistically "in control" or "out of control." Control charts are graphical approaches to fault detection where the sampled values of a parameter (or residual) are plotted with an expected distribution indicating the centerline value and upper and lower control limits (Figure 4.4). Two types of control charts are common: mean-standard deviation control chart $(\overline{\mathrm{X}}-\mathrm{S})$ and mean-range control chart $(\overline{\mathrm{X}}-\mathrm{R})$. These charts can be used when the system is relatively stable, meaning that the standard deviation (or range) does not vary significantly over time under normal operation. $\overline{\mathrm{X}}$-S chart is used when a large number of samples are available to calculate the standard deviation. When the sample size is too small to accurately estimate the variance, the $\overline{\mathrm{X}}-\mathrm{R}$ chart can be used considering the range to be an alternative to the standard deviation. Both the $\overline{\mathrm{X}}-\mathrm{S}$ and $\overline{\mathrm{X}}-\mathrm{R}$ charts are useful for detecting large changes ( $3 \sigma$ or more) in a process mean or variance, but they do not perform as well for small changes. The cumulative sum (CUSUM) chart can be used as a supplement to the traditional control charts to detect smaller changes (such as a $1 \sigma$ or $2 \sigma$ change in mean or variance). Control charts provide a heuristic approach to fault detection, as opposed to the hypothesis testing used in SPRT. Because of their graphical nature, the variation patterns created in control charts can sometimes be used for fault diagnostics. Several rule sets have been proposed to evaluate the type of error based on control chart patterns, including Nelson rules (Nelson 1984) and the Western Electric or Wheeler rules (Montgomery 2005). 


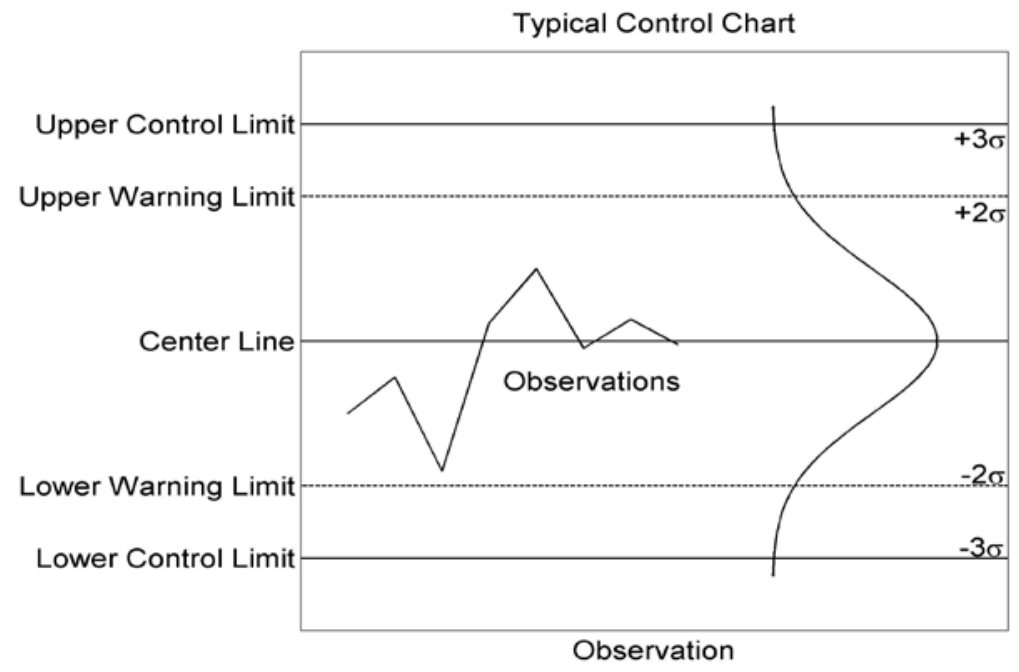

Figure 4.4. Control Chart for Fault Detection

The goal in fault (or flaw) detection in passive components is similar to the goal for active component fault detection - does a measurement contain potential evidence of a flaw in the material or component? As of this writing, flaw detection and diagnostics in most commercial applications of NDE is generally performed manually; that is, by one or more analysts examining the measured data to identify signals of interest, followed by a more comprehensive evaluation (also manual) of the measured data to determine if the signal is from a flaw of interest. The detection is also generally performed as a function of spatial location (as opposed to the time-based fault detection typically used with active components). However, the basic principles are the same in that, in both cases, some form of change detection approach is used.

In recent years, there has been increased interest in the use of automated algorithms to analyze NDE measurements from passive components. While most of the work is still in the research phase, there are some notable successes, where the automated analysis technology is being transitioned to use in a field setting (Zetec ; Benson et al. 2007; EPRI 2008a, 2009b). Automated algorithms for flaw detection are usually applied off-line, that is, after all of the measurement data has been acquired (though some recent applications in the nuclear power area are emerging where the goal is near-real time flaw detection (Benson et al. 2007; EPRI 2009b)). In general, automated analysis also follows a two-step process as in the manual case, where the first step is to identify signals of interest (detection) followed by a second step of careful analysis to determine if the identified signal is a flaw and, if so, to characterize the severity of the identified flaw. This section discusses the first (detection) step only; the second step is detailed in the next section of Fault Diagnostics. Note that, in the literature, such a division is often not explicitly described and, indeed, the line dividing the two steps is somewhat fuzzy at best. However, the distinction is made here to align the discussion on passive component fault diagnostics with that on active component diagnostics and maintain a uniform outline.

The problem of flaw detection in passive components has seen a number of approaches, which are usually empirical in nature. Typically some signal enhancement operations (prefiltering) are performed first (Udpa 2004) (either explicitly, or implicitly as part of the thresholding phase applied next). The goal here is to reduce the impact of measurement noise and enhance the overall signal-to-noise ratio (SNR). Often, this step also results in a reduced set of signals that need to be evaluated, as obvious non-flaw 
signals are eliminated. This is usually followed by some form of thresholding (either in the time domain or in an alternative domain such as frequency or wavelet) to clearly identify the signals of interest. The benefits of this approach are most often apparent in the analysis of imaging data, as this step can eliminate all but a few regions of the image for follow-on analysis (Xiang et al. 2001).

Approaches that have been proposed for detection are based on a range of filtering and transform algorithms, including adaptive filtering (for instance, Shekhar et al. 2001), template-based algorithms (for instance, Baskaran et al. 2004), time-frequency transforms (for instance, Legendre et al. 2000), and other transforms such as the Hilbert-Huang Transform (Quek et al. 2003). The diversity of approaches is partly due to the diversity in available nondestructive measurement techniques. In the area of ultrasonic NDE alone, a number of SNR enhancement techniques have been assessed. Spatial and frequency compounding (Bencharit et al. 1986; Choi 2007) methods use weighted averages of several measurements to improve SNR. Deconvolution methods assume a linear model for each of the measurement subsystems, and attempt to compensate for their effect on the measured data. Deterministic deconvolution techniques that assume a convolving filter did not result in an improvement in SNR with model data, particularly for low amplitude responses from cast austenitic stainless steel (CASS) specimens (Hargreaves 1988). Similar results were observed when deconvolution using a minimum phase filter was applied, and the most promising results were obtained by a minimum entropy deconvolution technique (Hargreaves 1988). Other studies have also indicated the potential for improvement in flaw detection through deconvolution (Ghouti 1997). Wavelet and related timefrequency techniques have also been successfully applied to reduce speckle noise and enhance ultrasonic signal SNR (Chen et al. 1999; Park et al. 2004). Other methods, such as the split spectrum processing technique (Bilgutay et al. 1989; Shankar et al. 1989) as well as the more general class of synthetic frequency diversity algorithms (Ericsson 1994) also show some potential. Time-averaged mean and mean-squared values were effective when the back surface response was relatively strong but were relatively ineffective for low amplitude signals (Hargreaves 1988). However, split-spectrum based polarity thresholding (Shankar et al. 1989), either alone or in combination with other processing techniques such as minimization (Newhouse et al. 1985) appears to significantly improve detectability in challenging materials, such as CASS specimens. A maximum entropy model of spectral analysis also had limited success (Hargreaves 1988). Miralles et al. (2004) discuss the application of higher order statistics (HOS) for analyzing ultrasonic backscatter. Though the focus of the work is on classifying scatterer (or grain) sizes based on HOS, similar approaches may potentially be applied to improve SNR or characterize microstructure. The use of nonlinear homomorphic filters (Morris et al. 1995) for reducing distortion and improving the imaging of strong scatterers have also been proposed. Note that synthetic aperture focusing techniques (SAFT) for CASS inspection (Silverstein and Thomas 1993; Anderson et al. 2007) also make use of signal processing tools to improve the beam-forming capabilities and reduce clutter.

Similar approaches to addressing the detection problem for other NDE measurements (Arunachalam et al. 2002; Ramuhalli et al. 2003b; Shin et al. 2004) techniques have been studied.

\subsection{Fault Diagnostics}

When a fault has been detected, diagnostic routines attempt to determine some information about the fault. Diagnosis can entail fault isolation, fault identification, or, most commonly, both. Fault isolation refers to locating the fault to a specific piece of equipment or area. Fault identification refers to determining the cause of the fault. Often, these two analyses are completed in tandem; a fault is detected 
and the diagnostic system determines both the location and cause (and, in some cases, severity) given the available fault symptoms. Fault symptoms are the features and signatures available that may help diagnose the fault; symptoms could include sensed data, monitoring system residuals, fault detection results, alarm patterns, etc.

Traditionally, diagnostics employed rule-based systems, wherein explicit if-then rules were used to determine a diagnosis given a symptom or set of symptoms. These systems were attractive, because they were naturally able to capture engineering judgment and past experience in a set of rules. However, rulebased systems are extremely brittle (Rich and Venkatasubramanian 1987). Before a new rule can be added, it must be compared to all the existing rules to avoid inconsistencies and conflicts (particularly when one symptom may be indicative of multiple different fault modes). Rule bases can easily become unmanageable as the rule list is expanded to include more and more scenarios. The if-then rules do not contain any deep knowledge of the system, so the rule-based engine will necessarily fail when a new condition is encountered that is not encapsulated in the rules, and changes in system design and operation are not easily propagated through the existing rules. Fuzzy rule-based systems overcome some of these challenges by naturally allowing a certain amount of uncertainty, or "fuzziness," in the rules; here, conflicting rules are not as detrimental to the overall system and "guesses" can be made for new situations based on the expert knowledge contained in rules. However, even fuzzy systems can suffer from rule bases that are too large for practical analysis. Research in diagnostic systems has investigated more sophisticated algorithms for several decades. A review of early work is given by Milne (1987).

In addition to expert systems, many traditional and advanced classification algorithms have been applied to fault diagnostics, including k-nearest neighbors ( $\mathrm{kNN})$, principal component analysis (PCA), neural networks, self-organizing maps, clustering, fuzzy clustering, etc. A series of review papers describes diagnostic methods based on quantitative models (Venkatasubramanian et al. 2003c), qualitative models (Venkatasubramanian et al. 2003a), and process history (Venkatasubramanian et al. 2003b). Yang (2004) gives a thorough review of both model-based diagnosis and data-driven diagnosis.

While diagnostic algorithms can be generally developed, fully developed diagnostic modules are extremely situationally dependent. A diagnostic system for a car engine is not applicable to a jet engine. In fact, a diagnostic system for a specific car engine (say a 1998 Saturn SL-1) may not be appropriate for a 1998 Ford Taurus, or even a 1995 Saturn of the same model. However, some work has been done in the power industry, specifically by the Electric Power Research Institute (EPRI), to pool information across a fleet of power-generating stations (both nuclear and non-nuclear) to build a more widely applicable Diagnostic Advisor. That work is briefly described in a later section.

The issue of diagnostics in passive components is similar, in that the goal is to determine whether a highlighted signal of interest (see previous section) contains evidence of material damage, and if so, whether the severity of the damage (in terms of location, size, and shape) can be quantified. This problem of material damage diagnostics is part of a general class of problems referred to as inverse problems (National Research Council 1996; Udpa and Udpa 1997; Ramuhalli 2002). Inverse problems in general are ill-posed (Tarantola 1987), lacking both uniqueness and continuous dependence of the measured signals on the inputs (Vogel 2002). This has resulted in the development of a variety of solution techniques for inverse problems in NDE (Udpa and Udpa 1997). As discussed in the previous section, flaw diagnostics for passive components in the field is generally performed by a manual analysis of measurement data. In the nuclear plant NDE area, this is almost exclusively the case although flaw detection in other application areas is slowly moving to the use of automated algorithms. Manual analysis 
typically makes use of a calibration standard. Measurements from the component under test are compared to those from the calibration standard to determine whether a response is flaw-like or not. In recent years, the use of data from a calibration standard has also been proposed for automated analysis and is an example of a direct approach for flaw diagnostics, which maps the measurement to the material property space (Hwang et al. 1997; Udpa and Udpa 1997; Ramuhalli et al. 2002; Sohn et al. 2003). Direct approaches apply a feature extraction step to extract relevant attributes of the measurement signal that has been identified as high interest during the detection phase. Physical features (such as rise time and energy), frequency-domain features, statistical features (high-order moments), and time-frequency features (wavelets, etc.) have all been applied to this task (Polikar et al. 1998; Simone et al. 2001; Kim et al. 2006). The final step of flaw signal detection is performed using one of several approaches, including hypothesis testing, neural networks, fuzzy systems, expert systems, etc., where the original measurement may be ultrasonic, radiographic, eddy current, or visual (or some other nondestructive measurement approach).

However, direct techniques are limited in that they require data from known damage (training data) to determine the mapping parameters, are sensitive to noise, and can only be used when measurements are acquired from flaws similar to those used in the training process. Alternative approaches draw inspiration from the many techniques that are well-established for inversion (or reconstruction) in the area of imaging (Barrett and Myes 2004).

In the context of flaw diagnostics (specifically quantification of flaw parameters such as shape and size), iterative methods that employ simulation models of the measurement physics are generally considered as an alternative to direct techniques (Yan et al. 1998). The model is used to estimate the measurement given the flaw or damage characteristics (i.e., shape, size, and location), which is iteratively derived by minimizing the difference between the estimated and actual measurements using optimization techniques such as conjugate gradient, simulated annealing (Kirkpatrick et al. 1983), or genetic algorithms (Haupt 1995).

Two classes of forward models (i.e., models of the measurement physics) have been utilized. The first group of models are numerical models such as a finite element model or integral equation models (Hoole et al. 1991; Caorsi et al. 1994; Monebhrrun et al. 1998; Yan et al. 1998; Balasubramanian et al. 2001; Li et al. 2001; Kim et al. 2003; Arunachalam 2006; Connolly et al. 2009), and although accurate, tend to be computationally expensive because the models must be solved during each iteration. Alternative forward models based on neural networks have also been proposed (Qing et al. 1997; Zhang and Gupta 2000; Ramuhalli et al. 2002, 2003a; Joshi 2006).

Other non-iterative forward model-based inversion techniques have also been proposed for the general problem of flaw diagnostics, including tomographic reconstruction using the Fourier slice theorem (Kim et al. 2003; Barrett and Myes 2004; Arunachalam 2006), point source technique, and (Potthast 2001) linear sampling (Colton and Kirsch 1996; Colton and Kress 1998). Constraints on material properties in the form of Markov random fields (Caorsi et al. 1994), as well as other regularization methods, have also been attempted (Tikhonov and Arsenin 1977; Morozov 1993; Vogel 2002; Barrett and Myes 2004). These methods are non-recursive (i.e., they attempt to solve the inverse problem over the entire problem domain using all of the data) and are generally computationally expensive. 
Data fusion algorithms (Waltz and Llinas 1990; Abidi 1992; Dasarathy 1997; Hall and Llinas 1997) for inverse problems in NDE have primarily been applied in direct solutions. In general, algorithms based on transform-based methods, Kalman filtering (and extended Kalman filtering) methods, Bayesian and other stochastic approaches, evidence-based techniques (such as Dempster-Shafer evidential reasoning), neural networks and rule-based systems have all been proposed for NDE applications (Pearson et al. 1988; Maren et al. 1989; Abidi 1992; Gros 1997; Liu et al. 2003; Ramuhalli and Liu 2004). Several authors have proposed the use of other techniques that include wavelet transforms (Verma et al. 2003; Kumar and Ramuhalli 2005b; Kumar et al. 2005), geometric transformations (Oagaro et al. 2004), independent component analysis (Simone and Morabito 2000), neural network algorithms (Yim et al. 1994; Ramuhalli and Liu 2004), and fusion using optimum filters or deconvolution methods (Yim et al. 1996; Kumar and Ramuhalli 2005a). There are relatively fewer methods that attempt to use statistical techniques for data fusion for NDE (Lee and Bajcsy 2004; Basseville et al. 2007). Applications range from NDE of aerospace components (Forsyth and Komorowski 2000; Liu et al. 2003; Kumar and Ramuhalli 2005b; Kumar et al. 2005) to thermal protection systems in aerospace vehicles (Hundhausen 2004) and gas transmission pipelines (Oagaro et al. 2004). Recent work in this area has focused on optimal fusion metrics for wavelet transform-based fusion algorithms (Kumar and Ramuhalli 2005b), generalized regression methods for multisensor fusion (Liu et al. 2008), and decision-level fusion (Dion et al. 2007), with applications for aerospace structures (Kumar and Ramuhalli 2005b; Kumar et al. 2005) and nuclear power plants (Kumar and Ramuhalli 2005a). Fusion using physics-based models, although not as widespread, has also been investigated by several authors (Nandhakumar and Aggarwal 1997; Tian et al. 2003).

One specific issue that has seen a greater focus in passive component diagnostics is the concept of reliability of the measurement technique. In general, the detection and diagnostics of flaws in materials is subject to uncertainty from a number of sources including measurement noise, material microstructure, surface condition and access, and human factors. A number of studies have been conducted to evaluate the reliability of the different NDE measurement methods and have resulted in probability of detection (POD) information (Berens and Hovey 1981, 1983) identifying the probability of detection of a flaw of specified size, false call probability (FCP), and the associated confidence bounds based on flaw type, material, and inspection technique (Singh 2000). In the nuclear power area, NDE reliability studies resulted in performance demonstration requirements that are codified in the ASME BPV Code (Section XI, Appendix VIII), and are used to qualify equipment, procedures, and personnel prior to allowing their use in ISI. The use of automated analysis methods for flaw detection and diagnostics adds a layer of complexity to the assessment of reliability. For instance, when using automated analysis methods, questions arise regarding the applicability of the algorithms to data that is generally not available during the algorithm development process. In recent years, EPRI has been evaluating the use of performance qualification procedures for automated analysis systems. A recent example is the Automated Analysis Performance Demonstration Database, which is used to qualify automated analysis tools for eddy current inspection of steam generator tubing (EPRI 2009b).

Whether using manual or automated analysis methods, the POD associated with a particular measurement and analysis technique will need to be incorporated in any subsequent remaining useful life analysis (Simonen et al. 2007; Kulkarni and Achenbach 2008). In turn, the failure probabilities can also be applied to determine the effectiveness of ISI programs (Khaleel and Simonen 2009). 


\subsection{Prognostic Algorithms}

As suggested by the "No Free Lunch" Theorem, no one prognostic algorithm is ideal for every situation (Ho and Pepyne 2001; Koppen 2004). A variety of models have been developed for application to specific situations or specific classes of systems. The efficacy of these algorithms for a new process or system depends on the type and quality of data available, the assumptions inherent in the algorithm, and the assumptions which can validly be made about the system. As such, these prognostic algorithms can be categorized according to many criteria. One proposed categorization focuses on the type of information used to make prognostic estimates; this results in three classes of prognostic algorithms (Figure 4.5) (Coble and Hines 2008; Hines and Usynin 2008).

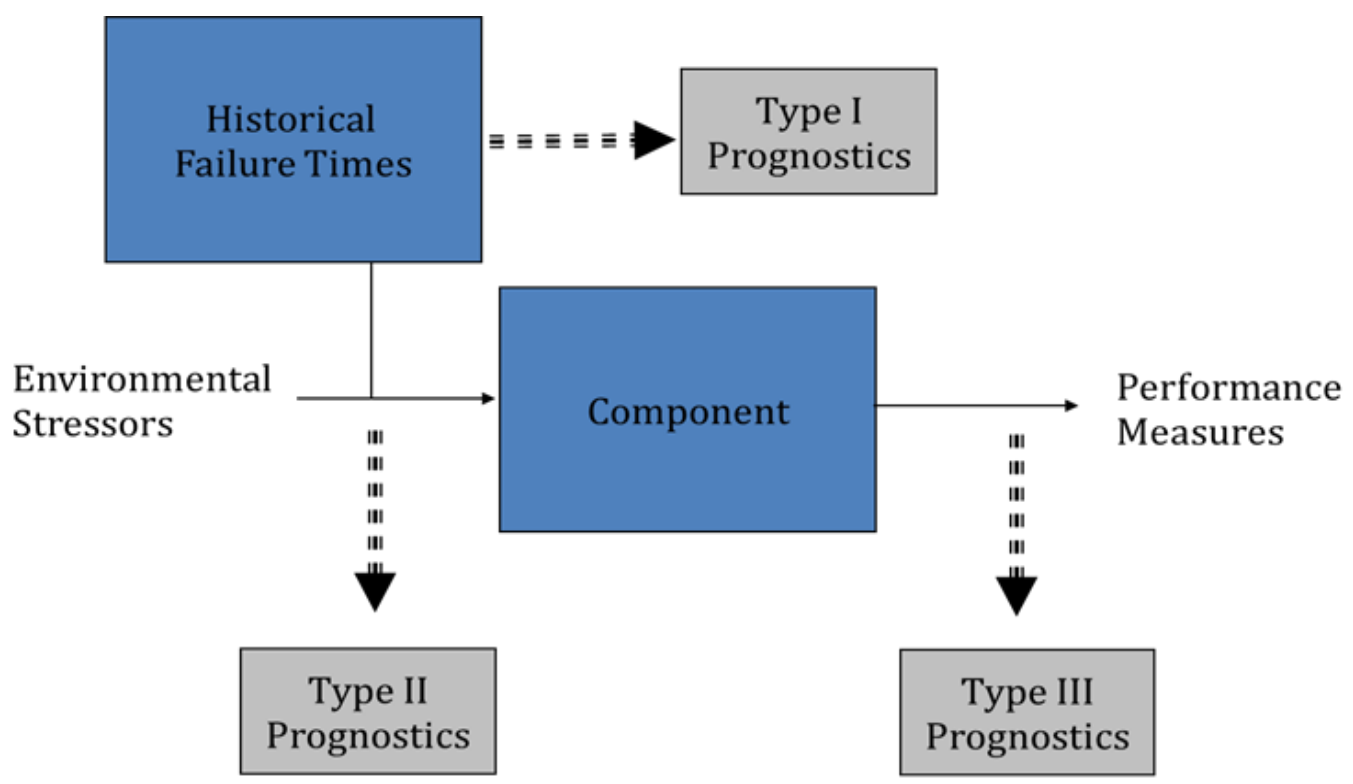

Figure 4.5. Prognostic Algorithm Categorization (Hines et al. 2008b)

Type I, or reliability-based, prognostics is traditional time-to-failure analysis; this type of prognostic algorithm characterizes the expected lifetime of an average system operating in an historically average environment. These methods may be applied if no data specific to the current system is available. Examples of Type I prognostics include Weibull analysis, exponential or normal distribution analysis, and nonparametric distribution analysis. A readily apparent shortcoming of this group of methods is the absence of consideration for operating conditions and environment in making RUL estimates. Typically, systems operating in harsher conditions will fail more quickly while those in milder environments more slowly.

Type II, or stressor-based, prognostics address this shortcoming by incorporating operational and environmental condition data to estimate RUL. This type of prognostics characterizes the lifetime of an average system or component operating in a specific environment. Type II methods can be used if operating conditions, such as load, input current and voltage, ambient temperature, vibration, etc., are measurable and correlated to system degradation. Algorithms in this class include simple regression analysis, specific formulations of the Markov Chain model and shock model, the proportional hazards 
model, and the life consumption model. Although more specific than Type I models, Type II models are deficient because they neglect unit-to-unit variance, which may be due to manufacturing-, installation-, and maintenance action-variability.

The final class of algorithms, Type III or degradation-based prognostics, characterize the lifetime of a specific unit or system operating in its specific environment. Extrapolation of a general path model (GPM) or a particle filter model is the most common empirical Type III method. This extrapolation involves trending a prognostic parameter and extrapolating it to some predefined failure threshold. A prognostic parameter is a measure, either directly sensed from the system or inferred from a set of sensor readings, which characterizes system degradation or health. System failure is commonly indicated by a soft failure threshold at which the system no longer performs to its specifications or cannot be expected to perform for an appreciable amount of time; this is generally some point before a catastrophic failure occurs. Additional Type III methods include a degradation-based formulation of the Markov Chain model and the shock model. This kind of individual-based analysis is generally considered the ultimate goal of prognostics for safety-critical or high-value components and systems.

Several surveys of prognostic algorithms are available (Schwabacher 2005; Schwabacher and Goebel 2007; Hines et al. 2008b), but the details of these algorithms are beyond the scope of this report. Some key algorithms in each of the prognostic categories are summarized in Table 4.3. Prognostic algorithms typically produce point estimates for RUL; however, estimates of the uncertainty associated with these RUL estimates are necessary to allow for optimized maintenance, operations planning, and safety when using RUL for risk-based decision-making. The following section briefly describes the research in characterizing prognostic uncertainty. This is followed by a description of prognostic performance metrics.

\subsubsection{Prognostic Uncertainty Analysis}

A systematic analysis of uncertainty can help reveal both reducible and irreducible uncertainty sources to aid in managing the overall RUL uncertainty. Uncertainty in prognostic estimates stems from several sources. Usynin (2007) identifies four sources: (1) variability of the severity and order of both past and future operating conditions (or loads), (2) variability in the initial degradation of a component or system, (3) variability in the amount of degradation which defines failure (the critical degradation threshold), and (4) data uncertainty due to inspection and data collection routines. Sankararaman et al. (2009) and Liang et al. (2009) identify two additional sources of uncertainty: physical variability (i.e., variability in material properties, manufacturing, geometry, etc.) and model uncertainty (i.e., model integrity and appropriateness, including uncertainty in how well the model captures the failure mechanism and uncertainty in the model parameters). The effect of model choice on prognostic model performance is investigated in Daigle et al. (2011) and Saha et al. (2011).

Several approaches have been proposed and applied to the problem of uncertainty estimation, each of which is well-suited to specific algorithms or systems. Closed-form equations for uncertainty estimation are used in Hines and Seibert (2006) and Engel et al. (2000) based on the prognostic model architecture. 
Table 4.3. Classification of Selected Prognostic Algorithms

\begin{tabular}{|c|c|c|c|c|c|}
\hline & Algorithm & Innuts & Assumntions & $\begin{array}{c}\text { Application } \\
\text { Snaces }\end{array}$ & Example References \\
\hline 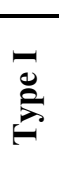 & Weibull Analysis & $\begin{array}{l}\text { Current } \\
\text { Runtime }\end{array}$ & $\begin{array}{l}\text { Component and system failure can be accurately } \\
\text { described by population-based failure probabilities }\end{array}$ & $\begin{array}{l}\text { Traditional } \\
\text { reliability } \\
\text { analysis }\end{array}$ & $\begin{array}{l}\text { (Yang and Xue 1996; Lall et al. 1997; Pecht } \\
\text { et al. 2002; Girish et al. 2003; Vichare et al. } \\
\text { 2004; Chen and Zheng 2005; Kharoufeh and } \\
\text { Cox 2005; Xu and Zhao 2005) }\end{array}$ \\
\hline \multirow{6}{*}{ ל. } & $\begin{array}{l}\text { Proportional } \\
\text { Hazards Models }\end{array}$ & \multirow{6}{*}{$\begin{array}{l}\text { Operating } \\
\text { History }\end{array}$} & $\begin{array}{l}\text { Uses operation condition-based covariates to } \\
\text { modify a baseline hazard function }\end{array}$ & & $\begin{array}{l}\text { (Cox and Oakes 1984; Dale 1985; Kumar } \\
\text { and Klefsjö 1994; Liao et al. 2006) }\end{array}$ \\
\hline & $\begin{array}{l}\text { Physics of Failure } \\
\text { Models }\end{array}$ & & $\begin{array}{l}\text { Accurate models of the underlying physical } \\
\text { progression of faults to failure must be available }\end{array}$ & $\begin{array}{l}\text { Electronics, } \\
\text { materials }\end{array}$ & $\begin{array}{l}\text { (Pecht and Dasgupta 1995; Kacprzynski et } \\
\text { al. 2002; Valentin et al. 2003; Kacprzynski } \\
\text { et al. 2004; Oja et al. 2007; Kulkarni and } \\
\text { Achenbach 2008) }\end{array}$ \\
\hline & $\begin{array}{l}\text { Life Consumption } \\
\text { Models }\end{array}$ & & $\begin{array}{l}\text { Operation at a given condition consumes some set } \\
\text { amount of life, which is subtracted from the } \\
\text { expected equipment life. }\end{array}$ & $\begin{array}{l}\text { Electronics, } \\
\text { circuit boards }\end{array}$ & $\begin{array}{l}\text { (Ramakrishnan and Pecht 2003; Mishra et } \\
\text { al. 2004) }\end{array}$ \\
\hline & Regression Analysis & & $\begin{array}{l}\text { Built-in Self Test (BIST) results are used to } \\
\text { extrapolate the effects of operation on the full } \\
\text { system }\end{array}$ & Circuit boards & $\begin{array}{l}\text { (Goodman 2000; Mishra et al. 2002; } \\
\text { Hofmeister et al. 2006a; Hofmeister et al. } \\
\text { 2006b) }\end{array}$ \\
\hline & $\begin{array}{l}\text { Markov Chain } \\
\text { Models }\end{array}$ & & $\begin{array}{l}\text { Future operating conditions are random and do not } \\
\text { depend on past conditions. A predictable amount of } \\
\text { damage occurs in each operating condition. }\end{array}$ & & \\
\hline & Shock Models & & $\begin{array}{l}\text { Shocks occur randomly in time and magnitude, } \\
\text { usually as a function of the current operating } \\
\text { conditions. }\end{array}$ & & \\
\hline \multirow{5}{*}{ छ } & General Path Model & \multirow{5}{*}{$\begin{array}{l}\text { Prognostic } \\
\text { Parameter }\end{array}$} & $\begin{array}{l}\text { A parametric model (regression, neural network, } \\
\text { etc.) is fitted to a prognostic parameter and } \\
\text { extrapolated to the failure threshold }\end{array}$ & & $\begin{array}{l}\text { (Upadhyaya et al. 1994; Chinnam 1999; } \\
\text { Engel et al. 2000; Byington et al. 2004; } \\
\text { Hines et al. 2006; Keller et al. 2006; Brown } \\
\text { et al. 2007; Coble 2010; Liu et al. 2010; } \\
\text { Coble and Hines 2011) }\end{array}$ \\
\hline & LEAP-Frog & & $\begin{array}{l}\text { Similar to the GPM using a short-window } \\
\text { regression for faster response to system changes }\end{array}$ & & $\begin{array}{l}\text { (Greitzer et al. 1999b; Greitzer 2001b; } \\
\text { Greitzer and Ferryman 2001) }\end{array}$ \\
\hline & Particle Filter & & $\begin{array}{l}\text { Model of damage accumulation is available as are } \\
\text { estimates of noise in the measurement. }\end{array}$ & $\begin{array}{l}\text { Passive } \\
\text { components }\end{array}$ & $\begin{array}{l}\text { (Orchard and Vachtsevanos 2007; } \\
\text { Ramuhalli et al. 2010) }\end{array}$ \\
\hline & $\begin{array}{l}\text { Markov Chain } \\
\text { Models }\end{array}$ & & $\begin{array}{l}\text { Similar to above, but the model is informed by the } \\
\text { current level of degradation }\end{array}$ & & \\
\hline & Shock Models & & $\begin{array}{l}\text { Similar to above, but shock arrival time and/or } \\
\text { magnitude is informed by the current amount of } \\
\text { degradation }\end{array}$ & & \\
\hline
\end{tabular}


Liu et al. (2010) utilize a bootstrap approach wherein the prognostic model is developed and executed many (in this work, 50) times and features of the RUL prediction are estimated from the aggregate results. Sankararaman et al. (2009) employ a Monte Carlo approach with a physical degradation model, a modified Paris' crack growth model. For each simulation, model parameters are randomly sampled from the parameter distributions, and the resulting crack growth is evaluated. The final result is a distribution of crack lengths as a function of the number of duty cycles.

Bayesian approaches to estimating uncertainty are common because they naturally incorporate information about the current system with prior knowledge (i.e., expert opinion, results of past analyses). Byington et al. (2004) utilize Bayesian belief models to estimate the uncertainty. Saha and Goebel (2008) utilize Bayesian methods for quantifying the uncertainty in both diagnostic (through relevance vector machines) and prognostic (through particle filters) results for battery monitoring. Liang et al. (2009) present two Bayesian-based uncertainty estimation methods: inner-outer loop Monte Carlo simulation for offline estimation of uncertainty and Bayesian estimation based on particle filtering models for online application. The authors further develop uncertainty analysis methods for additional filtering algorithms (particle filtering, exact filtering, and multiple-model filtering) based on the Fokker-Planck-Kolmorgorov (FPK) partial differential equation for uncertainty representation with dynamic system models (Liang et al. 2010a). This method can be applied to systems with exact solutions (exact filtering), systems that switch between competing fault modes (multiple-model filtering), and nonlinear systems excited by nonGaussian noise (particle filters).

An accurate estimate of RUL uncertainty is necessary to evaluate the efficacy and usefulness of a prognostic result. In addition to uncertainty, several prognostic performance metrics have been proposed in recent years, both for offline method evaluation and online RUL estimation evaluation. These are described in the next section.

\subsubsection{Prognostic Performance Metrics}

Performance metrics are necessary to allow model developers to compare two or more competing models, to understand the validity of a prognostic estimate, and to characterize model performance over different operating regimes, fault modes, or systems. Performance metrics for monitoring, fault detection, and diagnostic systems are well established (Hines et al. 2008a), including accuracy, robustness or autosensitivity, spill-over or cross-sensitivity, fault detectability, uncertainty measures, fault detection time, and false alarm/missed alarm rates. However, these conventional metrics fall short in characterizing prognostic model performance (Saxena et al. 2009a). Leão et al. (2008b) attempt to extend some of these metrics to prognostics, including false and missed alarm rates, but these prognostic metrics have seen limited application.

Generally, there are two classes of prognostic model performance analysis: offline and online. Offline performance metrics evaluate the prognostic algorithm, as a whole, applied to a specific component or class of components. Offline performance utilizes known ground-truth, such as actual failure times, to evaluate model performance. This type of analysis can be useful in the development stage for selecting between multiple competing prognostic models. Online analysis gives a measure of how well a prognostic model is performing in real time for a specific SSC that has not yet failed. Offline performance metrics are currently more commonly proposed and employed for evaluating prognostic algorithms. Metrics such as the "badness indicator" given by the probability integral test (PIT) (Leao et 
al. 2010; Leao et al. 2011) can be used to comprehensively evaluate prognostic algorithms and compare competing solutions. The badness indicator quantitatively characterizes the deviation of the empirical cumulative density function (CDF) from the expected CDF in the PIT analysis. By establishing confidence bounds and critical values for PIT (Leao et al. 2011), traditional hypothesis testing can be used to determine how a prognostic algorithm performs given the amount of training and validation data available. Additionally, Leao et al. (2008b) suggests a prognostics extension to the receiver operating characteristics, called the progROC curve, to help evaluate the trade-off between two conflicting prognostics goals.

Prognostic algorithm performance metrics tend to characterize performance in terms of either accuracy (estimation error) or precision (uncertainty). The field, however, is plagued by a problem common to many areas of prediction: The more precise the prognostic estimate, the less likely it is that this estimate will be correct. Practically, there is a trade-off between RUL accuracy and RUL precision; therefore, both features should be considered simultaneously.

Prognostic models result in a time-series of RUL estimates, and the performance requirements for these predictions vary throughout the life of the system. In general, we are willing to suffer large errors and uncertainties early in life if the prognostic performance improves as the system approaches failure (Line and Clements 2006; Saxena et al. 2008b; Saxena et al. 2009a). Traditional error measures do not account for these progressive acceptable accuracy and precision levels. Saxena et al. (2008b) suggest several metrics to account for this, the most interesting of which are the $\alpha-\lambda$ performance metrics for accuracy and precision. The $\alpha-\lambda$ performance dictates that the accuracy (or uncertainty) should be within some specified $\alpha^{*} 100 \%$ of the actual value within a relative distance, $\lambda$, to the actual failure, as shown in Figure 4.6. In this figure, $r_{*}^{l}$ is the actual RUL, and both lines marked $r^{l}$ (red and green) represent different RUL estimates. The shaded region indicates $20 \%$ error about the actual RUL. The $\alpha-\lambda$ performance is a binary true/false metric indicating the estimate is or is not within the specified tolerance at a given fraction of life. For the case shown, both estimates have an $\alpha-\lambda$ accuracy of "true" for $\alpha$ of 0.2 and $\lambda$ of 0.5 ; however, only the red estimate would have an $\alpha-\lambda$ performance of "true" for $\lambda$ of 0.9 . The Prognostic Horizon, also proposed in Saxena et al. (2008b), indicates the lead time between end of life (failure) and when the prognostic model first predicts failure to some specified performance (accuracy and/or precision). These metrics are expanded in Saxena et al. (2009b, 2010a; 2010b) to incorporate predicted RUL distribution information. The proposed metrics are largely visual, requiring evaluation of a graph of model performance, similar to that in Figure 4.6. Development of a single value to quantify this performance has not yet been reported.

In addition to concerns about the importance of correctly accounting for temporal needs, prognostic models that predict that failure will occur within a short time before actual failure are generally considered better than those that predict failure will occur in the same short time after the actual failure. RUL estimates greater than the actual remaining life leave room for unexpected failures and unplanned maintenance. Saxena et al. (2008b) suggest an exponentially weighted accuracy metric to account for this, which gives a larger penalty for late predictions than for early predictions of failure. This metric considers the RUL predictions made at one point in time across a population of systems, instead of the entire time series of predictions. A similar error metric was used in the 2008 PHM data challenge (Saxena et al. 2008a). 


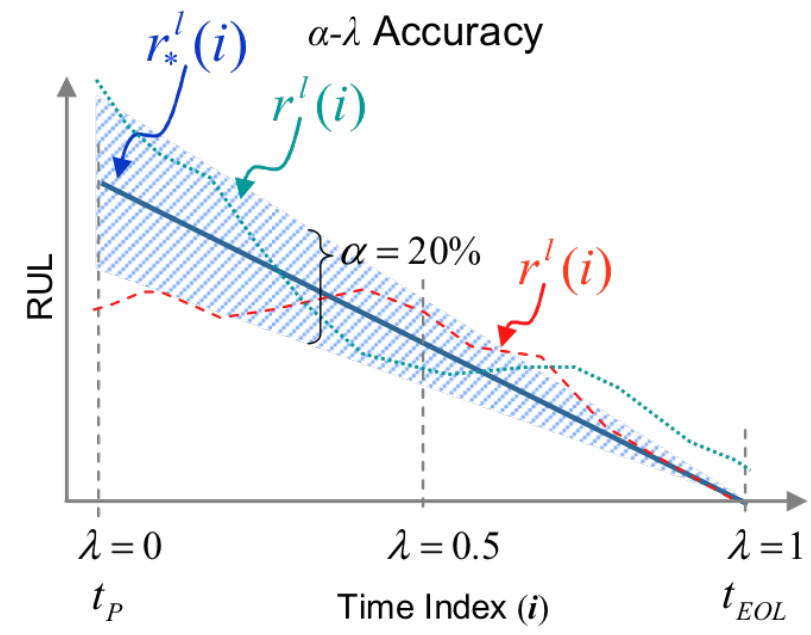

Figure 4.6. $\alpha-\lambda$ Performance for Accuracy (Saxena et al. 2008b)

Research in health monitoring algorithms and applications is active in many areas outside of the nuclear industry. In fact, most advances in PHM have originated in other areas, although the results may be applicable to NPPs. The following section summarizes the recent research in a few key industries: electronics, defense, avionics, and wind turbines. 


\subsection{Applications of Current Technologies in Other Fields}

Increasing availability and reliability is economically attractive for many applications and systems, while avoiding serious degradation and consequent failure is paramount for safety critical applications. As such, PHM and CBM have found use in a variety of fields in the past decades. For instance, automated CBM and control for building systems, such as heating, ventilating, air conditioning, and refrigeration (HVAC\&R) systems, can save an estimated 15-30\% of wasted energy in commercial buildings; a two-part review of these systems is given in Katipamula and Brambley (2005a, b). At the time of this review, automated CBM for building systems remained largely the purview of research. The authors cite several research needs to bring these systems to deployment, including well-defined methods for automation of fault detection, diagnostics, and control and methods to detect and diagnose multiple, simultaneous faults. Additionally, research at Sun Microsystems has led to the development of a continuous system telemetry harness (CSTH) for enterprise servers. The CSTH monitors parameters of the operating server, such as voltage, current, and temperature, and uses the MSET algorithm and SPRT detection routine to detect deviations from the expected server behavior (Gross et al. 2003; Mishra and Gross 2003; Whisnant et al. 2005; Gross et al. 2006). The use of CSTH data with electronic PHM algorithms has improved visibility of server health, reduced the number of Could-Not-Duplicate faults, and increased server availability.

The following sections summarize research and development in condition monitoring and PHM for electronics, defense systems, avionics, and wind turbines, which represent some of the most developed PHM application areas.

\subsection{Electronic Prognostics}

Traditionally, electronic failures were viewed as completely random in nature; however, significant research has looked at monitoring aging mechanisms and effects in recent years (Droste and Finklea 2006; Hofmeister et al. 2006b; Vichare and Pecht 2006; Gu et al. 2007; Kalgren et al. 2007; Bailey et al. 2011; Lall et al. 2011). Electronic system prognostics on the board or circuit level commonly utilize a built-in self test (BIST) prognostic monitor or canary (Goodman 2000; Mishra et al. 2002; Goodman et al. 2006; Hofmeister et al. 2006a). A prognostic monitor is a "pre-calibrated semiconductor cell that is colocated with the actual circuit on a semi-conductor device" (Mishra et al. 2002). The prognostic cell is designed to experience a higher current level than the actual circuit by decreasing the cross sectional area of the current-carrying path in the canary. Because the canary cell undergoes a higher current density, it is expected to fail in a predictably faster way than the actual circuit. By locating several prognostic monitors with different known accelerating factors on a circuit, the failure times of each of these cells can be trended to predict failure in the actual circuit at any time after at least two prognostic monitors have failed. While this method is convenient and uncomplicated, Pecht et al. (2001) argue that BIST monitors are not always sufficient for detecting and identifying failures. The authors found BIST results to suffer from a high false alarm rate and a low correlation between the fault indicated by the BIST and the actual fault. These shortcomings should be considered before applying this type of prognostic monitoring module.

The use of physics of failure models for estimating RUL has focused mainly on electronic system prognostics, because accurate physical models of component-level electronics are well-understood (Pecht and Dasgupta 1995; Kelkar et al. 1997; Valentin et al. 2003; Oja et al. 2007). Life Consumption models 
(LCM) based on physics-of-failure models were first proposed by Ramakrishnan and Pecht (2003) for monitoring RUL in electronic systems. The LCM methodology monitors the environment of a component or system during its entire lifecycle to determine the amount of damage incurred by the various loads and conditions experienced. This damage is translated to lost "life" which is subtracted from the expected life of an average system or component. LCM is illustrated in Ramakrishnan and Pecht (2003) and Mishra et al. (2004) by application to a mounted printed circuit board operated under the hood of a moving vehicle. Both temperature and vibration levels were monitored on the board during use. The methodology was shown to effectively estimate RUL of the circuit board, even in the event of unexpected damage accumulation caused by a large, random shock. Development of a more general LCM methodology which utilizes empirical models, such as neural networks, kernel regression models, or simple regression, for damage estimation would increase the applicability of this algorithm. Several methods for fusing physics-of-failure models and empirical results for electronic prognostics have been proposed (Mathew et al. 2008; Cheng and Pecht 2009).

\subsection{Defense}

PHM systems have found significant use in mission critical defense systems. The relevant work falls under the umbrella of several acronyms in addition to PHM: vehicle health management (VHM), integrated vehicle health management (IVHM), integrated structural health management (ISHM), health and usage monitoring systems (HUMS), etc. These programs share similar goals and technologies, but are often applied to different platforms. The ultimate goal for defense system PHM is to increase the likelihood of mission success through an autonomous real-time health monitoring system which supports automatic fault detection and diagnosis, failure prognosis, reconfiguration (when possible), and reporting to operators, maintainers, and logistics planners. Walker (2010) outlines a methodology for leveraging domain knowledge developed during initial design analysis (such as failure mode, effects, and criticality analysis [FMECA]) to develop a PHM system for remotely piloted aircraft, though the development methodology is more widely applicable to PHM system design for any system. Similarly, Line and Clements (2005) describe a systematic approach for developing PHM systems using the PHM Pro tool with application to the Joint Strike Fighter.

Significant efforts in defense system PHM coincided with the Joint Strike Fighter (JSF) program (Smith et al. 1997; Ferrell 1999, 2000). JSF was conceived as a platform for Autonomic Logistics, and PHM was a key technology to support that concept (Hess and Fila 2002; Hess et al. 2004). Autonomic Logistics, or AutoLog, replaces human-led logistics with automated flight scheduling, maintenance planning, and spare parts ordering informed by an assessment of the actual condition of critical components in operation. AutoLog relies on an integrated report from the PHM system to perform logistics planning; this automated approach was expected to minimize incorrect maintenance actions; decrease downtime during fault detection, troubleshooting, diagnosis, spare parts ordering, etc.; and reduce support response requirements. Additionally, by utilizing AutoLog across the entire fleet of JSFs, systemic problems could be identified and corrected before they become fleet-wide and catastrophic.

While there was much discussion of integrated PHM systems, related research for JSF largely focused on PHM solutions for specific subsystems or components, such as electronics and power supplies (Orsagh et al. 2005; Keller et al. 2006; Orsagh et al. 2006; Line et al. 2007), rotating components in jet engines (Suarez et al. 2004), propulsion systems (Powrie and Novis 2006), oil system monitoring (Powrie 2000), corrosion monitoring (Demo et al. 2011), etc. 
Research to support PHM for the M1 Abrams main battle tank gas turbine engine resulted in the development of a fault diagnosis engine called the Turbine Engine Diagnostics using Artificial Neural Networks (TEDANN) (Illi et al. 1994; Kangas et al. 1994; Greitzer et al. 1997; Greitzer et al. 1999a). This system used 48 sensors mounted on the engine (32 embedded sensors and 16 retrofitted sensors) to monitor and diagnose common engine faults. An unsupervised, self-organizing neural network was used to classify engine operation (i.e., low-idle, tactical idle, full power, etc.). A bank of supervised networks was used for engine modeling and diagnostics through pattern recognition. This system was later expanded into the Real-time Engine Diagnostics-Prognostics (REDI-PRO) system to incorporate a larger diagnostic capability and prognostics (Greitzer and Pawlowski 2002). REDI-PRO used information from 38 engine-mounted sensors ( 25 embedded and 13 retrofitted) with neural networks, rule-based diagnosers, and extrapolation-based prognosers. REDI-PRO prototype system was installed on two U.S. Army National Guard tanks for several years and on U.S. Army tanks at Yuma Proving Ground (YPG) for several months. While in place at YPG, one of the outfitted tanks experienced a failure caused by an extreme air filter clog. REDI-PRO was able to detect a change in the system and predict the imminent failure several operating hours before the detection of the air filter clog by onsite maintenance personnel (Greitzer and Ferryman 2001). While the specific neural networks and rule bases developed in this research are specific to the AGT1500 engine, the methodologies and architecture employed in REDI-PRO can also be applied to other systems.

The Advanced Technology Office (ATO) began development of a model-based dynamic diagnostic reasoning technology (DARTS) in the early 1990s (Su et al. 1993; Darty et al. 1994), which evolved into the Diagnostician (Giordano and Levy 1994; Giordano and Carey 1995). The Diagnostician has been successfully embedded in several systems (NAVSTAR, Seawolf Submarine, NASA Remote Power Controller) to provide more accurate diagnostics by extending the existing built-in test capabilities to built-in diagnostics. In the late 1990s, the Diagnostician was extended to including prognostic capabilities, called the Prognostics Framework (Su et al. 1999; Su et al. 2000b). The Prognostics Framework gives an overall system view of prognosis by using model-based reasoning to integrate builtin test and sensor data into diagnostic and prognostic models. It integrates the outputs of specialized prognostic modules for specific subsystems into one holistic view of system health and RUL. The Abrams M1A1 tank was a test platform for the Prognostics Framework (Su et al. 2000a). This test integrated the results of prognostic algorithms developed for five subsystems of the tank, including REDI$\mathrm{PRO}$, the gas turbine engine prognostic routine.

\subsection{Avionics and Aerospace Systems}

Many of the PHM technologies developed for defense systems can be leveraged in the commercial avionics and aerospace fields (Roemer et al. 2001; Smeulers et al. 2002; Fang and Guanzhong 2010). SmartSignal Corp. has provided fault monitoring services through their Equipment Condition Monitoring (eCM) tool to commercial airlines, including Delta Airlines and Southwest Airlines, since the early 2000s (Medill 2003). The algorithms used in eCM are based on the MSET model developed at Argonne National Laboratory for monitoring NPPs. Researchers at NASA are particularly interested in PHM and automatic reconfiguration for space vehicles, from launch site ground equipment (Ren et al. 2011) to reconfigurable control and mission planning (Liang et al. 2010b) to unmanned missions (Reichard et al. 2007). 
NASA developed a comprehensive roadmap for integrated vehicle health management to guide efforts in-house and coordinating efforts with other groups (Srivastava et al. 2009). This roadmap outlines an approach to developing PHM systems at four key levels. The first level is foundational, which includes development of advanced sensors and materials, advanced modeling (both physical and empirical), advanced analytics, and development of verification and validation routines. Level two includes subsystems such as the airframe, the propulsion system, and software. Level three focuses on themes, or programmatic research such as detection, diagnosis, prognosis, fault and failure mitigation, and integrity assurance. The final level combines the results and applications of the lower level into a validated, integrated vehicle health management system for the full aircraft. For each major level of research, the roadmap highlights key projects, milestones, and metrics that will be necessary to fulfill the needs of that research. This comprehensive technical plan provides a strong foundation for coordinating efforts across multiple groups and moving the state of aircraft PHM forward toward a final, deployable system.

\subsection{Wind Turbines}

The wide use of large wind turbines, also called wind energy convertors (WECs), has made wind energy the fastest growing renewable source of electricity generation worldwide. Onshore WECs achieve availability in excess of 95\% (Wilkinson et al. 2007); however, offshore deployment of WECs is increasingly attractive due to more favorable wind conditions offshore and reduced public objection to offshore location. Offshore location will significantly limit access to WECs for inspection and maintenance, which increases the emphasis on early detection and diagnosis of incipient failures (Wilkinson et al. 2006). Typically, scheduled maintenance of WECs is performed once every six months and requires approximately 24 hours to complete. Unscheduled maintenance due to unanticipated faults and failures requires, on average, 130 hours to complete (Adams et al. 2011). For offshore locations, the downtime for unscheduled maintenance may be even longer, while personnel wait for access to the site. To improve the economic competitiveness of wind energy, much research has focused on condition monitoring and diagnostics for subsystems, such as the drivetrain, rotors and blades, support structure, etc., and the WEC as a whole; reviews of related research are given in Hyers et al. (2006) and Hameed et al. (2009).

Most WEC monitoring systems rely on the available data from the Supervisory Control and Data Acquisition (SCADA) system, including wind parameters (speed and direction), energy conversion parameters (power output, rotor speed, generator torque, etc.), vibration parameters (drivetrain and tower acceleration), and temperature parameters (both component and air temperature) (Yang et al. 2009; Zaher et al. 2009). In Yang et al. (2009), experiments performed on a wind turbine condition monitoring test rig indicated that drivetrain mechanical faults could be detected by analyzing electrical signals from the generator, the power signal in particular, through the use of wavelet transforms. This method eliminates the need for costly torque, vibration, or proximeter measurements. Kusiak and Li (2011) developed a monitoring system which first detects a fault, then estimates the severity of the fault, and finally diagnoses the fault type. Using data collected by the SCADA system at 5-minute sampling intervals, faults were detected approximately 60-minutes before failure with reasonable accuracy. This is likely not a long enough lead time for offshore applications; however, the method may be improved by increasing the sampling speed of the SCADA system. Zaher et al. (2009) used neural networks to model nominal WEC behavior and used model residuals to detect anomalous behavior. The authors were able to detect a fault in the cooling oil six months in advance of a failure, which would give ample time to plan and execute 
maintenance actions to avoid the failure. They also suggest a multi-agent system (MAS) for integrating monitoring methods for multiple types of faults into one system, giving an overall WEC monitoring platform. Several commercial monitoring systems have been developed for WECs. Seven commercially available systems are described in Hameed et al. (2010).

Development and application of PHM technologies has been more active in non-nuclear fields over the past decades. However, significant work has been underway to advance the state of the art for nuclear applications, including health monitoring for NPP SSCs. The following section summarizes the recent work in developing PHM for application in NPPs. The state of the art is largely focused on fault detection and diagnostics for both active and passive components, although some work in prognostics is described. Additionally, the results of pilot applications and fielded systems in NPPs are summarized. 



\subsection{PHM Systems for Nuclear Power Applications}

Major progress is being made by early adopters in NPPs, such as by Electricity de France (EDF) and by Exelon, who are deploying 700 units with centralized diagnostic monitoring. In general, however, the nuclear power community is slower to adopt on-line health monitoring than other communities, particularly when compared with the fields reviewed earlier. Although PHM systems have not found ubiquitous use in NPPs, significant work has been completed to develop these capabilities. Reviews of the research in on-line monitoring (OLM) and PHM highlight several possible applications. Uhrig and Hines (2005) review the uses of computation intelligence in nuclear engineering, include noise analysis techniques, online sensor validation, regularization of ill-posed surveillance and diagnostic problems, transient identification, core monitoring, efficiency improvement, and anticipatory control. An early review of possible applications of OLM in NPPs was given by Hashemian and Feltus (2006), including identifying process-to-sensor problems, detecting instrument anomalies (such as venturi meter fouling), and detecting thermal hydraulic anomalies in the coolant system. Hashemian (2011c) further lists eight applications for OLM in NPPs: detecting sensing-line blockages, testing the response time of pressure transmitters, monitoring calibration of pressure transmitters on-line, cross-calibrating temperature sensors in situ, assessing equipment condition, performing predictive maintenance of reactor internals, monitoring fluid flow, and extending the life of neutron detectors. Ma and Jiang (2011) add instrument channel dynamic performance monitoring, reactor core monitoring, loose parts monitoring, and transient identification.

A recent review by an International Atomic Energy Agency (IAEA) Coordinated Research Programme (CRP) investigated and summarized the state of the art in advanced surveillance, diagnostic, and prognostic techniques for NPP SSCs (IAEA 2012). The applicable work in PHM can be divided between active and passive components, listed in Table 6.1. The following sections summarize the advances in PHM for active and passive components, respectively. The results of some pilot applications and field tests are then summarized.

Table 6.1. Examples of Active and Passive SSCs in NPPs

\begin{tabular}{cc}
\hline Passive SSCs & Active SSCs \\
\hline cables and connections & air compressors \\
containment & batteries \\
containment liner & circuit boards \\
core shroud & control rod drive \\
heat exchangers & cooling fans \\
piping & diesel generators \\
pressurizer & instrumentation \\
& channels (sensors) \\
pump casings & motors \\
reactor vessel & pumps \\
steam generators & transistors \\
support structures & valves \\
transformers & power supplies \\
valve bodies & \\
ventilation ducts & \\
\hline
\end{tabular}




\subsection{Active Components}

Many active components can be monitored through the data already sensed for the plant I\&C system. In some cases, additional continuous measurements will greatly improve monitoring ability, such as vibration measurements for pump monitoring. However, the detection and diagnosis of faults in interconnected systems is highly complex. Simply monitoring and thresholding sensed measurements is an incomplete approach to condition monitoring. The control systems related to most of the sensed values will work to keep them within specified tolerances through feedback loops. In many cases, it is necessary to consider fault detection and diagnostics at the system level instead of the device level by developing models of normal system behavior and comparing measurements to these model predictions. The following sections describe research to date on monitoring some key active components: sensors, pumps, and valves.

\subsubsection{Sensor Calibration Monitoring}

To ensure safe and effective operation of an NPP, sensing and transmitting reliable values for key parameters are central to nuclear power instrumentation for control. Current practice in the United States requires recalibration of process instrumentation channels during every refueling outage. These calibrations often require that the instrument be taken out of service and falsely-loaded to simulate inservice conditions (Hines et al. 1996). Recalibration is both a labor-intensive and costly process, resulting in longer outages, increased maintenance cost, and additional radiation exposure to maintenance personnel, and it can actually be counterproductive, introducing errors in calibration of previously unfaulted sensors. Hashemian (1995) describes methods to test calibration of sensors during steady state plant operation, including redundant channel averaging, process modeling, and comparison with calibrated reference channels. In 1998, EPRI demonstrated a non-intrusive method for monitoring the performance of instrument channels and extending the required recalibration interval for well-performing sensors (EPRI 1998). The NRC endorsed the generic concept of online monitoring for sensor calibration interval extension in 2000 and gave 14 requirements to be addressed by plant-specific license amendments necessary to relax the calibration frequency currently required by the technical specifications (NRC 2000). To date, the Sizewell B plant in the United Kingdom is the only NPP to receive approval for transmitter calibration extension from their regulatory authority (Lillis and Orme 2005). Rather than develop and propose a specific algorithm or set of algorithms for OLM, the burden of Sizewell B's safety case is centered around historical evidence from their maintenance records showing that their transmitters do not systematically drift. In this approach, OLM algorithms are used as a performance monitoring tool to provide more frequent monitoring of transmitter performance than the traditional manual calibration method, which only assesses transmitter performance at every refueling outage. Sensor calibration monitoring has shown at Sizewell B that sensors and transmitters may function reliably and within calibration for 8 years or longer (Hashemian et al. 2004; Lillis 2010).

The state of the art in OLM for sensor calibration interval extension was reviewed in the NUREG/CR-6895 series (Hines and Seibert 2006; Hines et al. 2008a; Hines et al. 2008c) and a recent IAEA report (IAEA 2008a). Research in monitoring algorithms has largely focused on empirical models, including neural networks (Eryurek and Turkcan 1991; Hines et al. 1996; Nabeshima et al. 2002; Ayaz et al. 2003; Seker et al. 2003; Fantoni 2005), multivariate state estimation technique (MSET) (Singer et al. 1995; Gross et al. 1997; Singer et al. 1997; Hines and Usynin 2005), and auto-associative kernel regression (Garvey et al. 2006; Garvey and Hines 2006; Garvey et al. 2007a, b). The results from pilot 
applications of OLM for calibration monitoring show that common sensor faults, including sensor drift, sensor bias, stuck sensors, etc., can be reliably detected (Bickford et al. 2002; Davis et al. 2002; Hines and Davis 2005).

The application of OLM for sensor calibration interval extension requires an amendment to the plant technical specifications (TS). To date, no U.S. plant has successfully obtained this amendment, leaving significant uncertainty in what will be necessary for a successful application. To alleviate some of this uncertainty, the Pressurized Water Reactor Owner's Group (PWROG) is working to develop generic criteria for the extension of calibration intervals and the associated license amendment. The research initiated by the PWROG will address several issues:

1. Determine the impact of transmitter calibration extension on probabilistic risk analysis (PRA), defense-in-depth, and safety margins.

2. Perform a generic transmitter drift study using statistical analysis of transmitter maintenance records.

3. Develop guidance to determine OLM acceptance criteria.

\subsubsection{Pumps}

The degradation and failure of reactor coolant pumps (RCPs) can cause significant economic losses for NPPs which have to shut down for extended periods for maintenance and replacement. As such, RCPs have received significant research attention for fault detection and diagnosis. Jenkins (1985) describes the expert analysis of the cause of increasing vibrations in the $1 \mathrm{~B} \mathrm{RCP}$ at Three Mile Island Unit \#1. This analysis involved removing the pump from service and performing a series of tests and first-principle analyses to identify the root-cause of the problem, which was eventually determined to be a cracked pump shaft in the shaft overhang below the pump bearing. The analysis was initiated when pump vibration levels reached 750 microns, a three-fold increase over the normal vibration range of 225-280 microns. The development of online fault detection and diagnostic systems could significantly reduce the burden on this type of prescribed testing and expert physical analysis, reducing maintenance time and preventing secondary damage due to the possible significant vibrations.

Singer et al. (1990) developed a SPRT-based fault detection and diagnostic system that was applied to the RCPs at the EBR-II reactor at Argonne National Laboratory. This monitoring system used a network of SPRT tests and if-then rules to (1) detect anomalies in the plant data in real-time and (2) determine if the anomaly was due to sensor or pump degradation. The developed system provided extremely early operator notification of anomalies, diagnosed the most likely fault mode, and recommended corrective actions based on existing operational and emergency procedures. A similar system using SPRT with an expert system for monitoring RCPs based on sensed speed, vibration, power, and discharge pressure measurements was patented by Gross et al. (1993).

Vibration monitoring is common for detecting and diagnosing faults in pumps. Commonly, vibration data are analyzed using the Fourier transform, but this method is only useful for stationary signals. Often, the statistical properties of signals collected from faulted equipment are changing due to the nature of the fault progression. To better capture and understand the time-dependence of these signals, a timefrequency representation is necessary. Koo and Kim (2000) use the Wigner distribution of the vibration signals to extract frequency information for fault detection and diagnostics. They use a single neural network trained to classify behavior as either normal or one of six faulted conditions: shaft bow, shaft 
misalignment, unbalance, oil-less bearing rub, structure looseness, and shaft crack. Jung and Seong (2006) use the Wigner-Ville distribution, which accounts for cross-interference between different components of a signal, to detect RCP impeller looseness. This approach makes use of power line signals, three-phase voltage, and current, instead of vibration signals. The three-phase voltage is measured from a secondary tap of potential transformer, and the current from current transformer output; this allows a non-intrusive measurement that will not interrupt plant operation. The proposed detection method was validated using data collected during heat-up and cool-down phases of operation. The authors corroborate their fault detection results through comparison to RCP vibration monitoring results and visual inspection.

Jarrell et al. (2004) identify the primary stressors which cause degradation of centrifugal pumps and formulate deterministic relationships between these stressors and the resulting degradation. Vibration data analyses showed agreement between the motor position indicator, the vibration response, and the dynamic force loading on the bearing in experimental tests. The experiments highlighted a set of correlations that link measurable degradation stressors to resulting degradation rates and failure.

\subsubsection{Valves}

Check valves are critical to safe NPP operation. The NRC has mandated periodic inspection of these valves, which typically involves partial disassembly. Haynes (1990) gives a review of monitoring and diagnostic methods for check valves, focusing on acoustic emission, ultrasonic inspection, and magnetic flux signature analysis. The state of the art at the time indicated that the combination of acoustic emission with either ultrasonic inspection or magnetic flux signature analysis would enable monitoring of all major check valve operating conditions. The reviewed technologies largely involved portable, walk-around monitors. Several studies have looked at using acoustic emission signals for valve health monitoring. Nakamura and Terada (1985) used high frequency $(100 \mathrm{kHz}+)$ resonance type acoustic emission sensors to monitor for leaks in pressurizer valves. After accounting for low levels of background noise, the acoustic signal was used to detect leaks and estimate the valve leak rate. Log-linear relationships between the RMS sensor output voltage and the valve leak rate were empirically defined for both subcooled water and saturated steam. Lee et al. (2006) took a similar approach to monitoring check valves for two types of failure: disk wear and foreign object intrusion. They developed linear relationships between the RMS output voltage and the leak rate dependent on the amount of disk wear or size of the foreign object. Furthermore, they were able to perform fault diagnosis based on the frequency spectra profiles of the check valve leakage. These spectra profiles are not highly dependent on pressure or leak rate, but are strongly dependent on the type of fault, exhibiting spectra peaks at different frequencies depending on the fault mode. McShane and Ulerich (1992) developed and patented a check valve monitoring system using ultrasonic transducers coupled to a pipe, instead of directly to the valve. This technique measured the fluid turbulence downstream of the valve to assess the condition of the check valve.

Classical methods for monitoring motor operated valves (MOVs) include off-line operator testing of valves during outages and remote testing methods that rely on accurate first principle models of the induction machine in the MOV. Chai et al. (1994) developed a first principle model of the motor in an MOV using measureable stator variables. An inverse filter was used to reconstruct the forces acting on the motor and actuator from vibration signals collected on the actuator casing, and an adaptive filter was used to adjust the gear mesh center frequency as the load changes and the speed of the motor varies. The developed models accurately estimated motor behavior and pinion and worm gear frequency for normal 
operation in laboratory tests. It was proposed that these accurate estimations of MOV forces and motions could be used in a diagnostic system; however, none was presented. Arcella et al. (1994) patented an online valve diagnostic monitoring system which relies on three sensors: a motor current sensor and strain and position sensors coupled to the valve stem. This system utilized some simple data analysis, such as deviations from baseline data and an expert rule-based system for fault diagnosis. Granjon (2011) proposes a method to monitor MOVs relying exclusively on remote electrical measurements, such as supply voltages and currents, without incorporating any internal MOV measurements. By replacing periodic walk-around monitoring with remote electrical monitoring, the MOV can be continuously monitored for degradation and faults. The estimated active power is used as an indicator of the stem nut mechanical condition. In this work, one indicator (active power) is used to monitor one component of the MOV. However, the results indicate that active power is closely related to internal mechanical phenomena in the MOV; this indicator may be useful for monitoring additional faults and/or components.

Upadhyaya et al. (2003) give an example of an advanced surveillance, fault detection, and diagnostic system applied to a turbine control valve during transient operation. This approach involves a data-driven model for predicting nominal variable values and a decision-making module for anomaly detection and diagnosis. The work presented is based on a full-scope PWR simulation code.

\subsection{Passive Components}

A key element of long-term operations of current and future NPPs is the management of aging and degradation in the materials and components that make up passive systems. Methods to assess the condition of these SSCs in a nondestructive way are necessary to assure adequate safety margins throughout plant life. Table 6.2 lists the technologies currently approved by the ASME Boiler and Pressure Vessel code for nondestructive inspection of nuclear structures and components. All of these, with the exception of AE, are allowed for periodic ISI. Acoustic emission monitoring is currently the only method sanctioned within the ASME Code for online monitoring of NPP structures and may only be used to monitor the growth of an existing flaw that has been characterized using another NDE technique. Evaluation of the growth rate based on these measurements must be performed every two months and extrapolated to the next outage to determine if mitigating or repair activities are necessary or if operation can continue uninterrupted. In addition, existing code rules imply that if a new flaw is detected by acoustic emission, the flaw must be sized using an alternative NDE method.

Table 6.2. Summary of NDE Technologies Included in Section XI of the ASME Boiler and Pressure Vessel Code

\begin{tabular}{ll}
\hline \multicolumn{1}{c}{ Surface Examination } & \multicolumn{1}{c}{ Volumetric Examination } \\
\hline Magnetic Particle & Radiographic \\
Liquid Penetrant & Ultrasonic Testing \\
Eddy Current & Eddy Current \\
Ultrasonic Testing & Acoustic Emission \\
Visual Inspection & \\
\hline
\end{tabular}


As existing plants consider extending their operating licenses beyond 60 years, the resulting increased material exposure to time at temperature, neutron irradiation, reactor coolant, and mechanical stress is likely to impact degradation accumulation in passive SSCs. Future plant designs will also likely begin service with longer expected lifetimes, leading to these same increased exposures. Experience with operating LWRs has shown that new degradation mechanisms appear in passive materials approximately every seven years (Wilkowski et al. 2002). Materials aging issues related to future plant designs are still not completely identified and will depend on the operating conditions as well as the specific material used. Monitoring and understanding operation and degradation conditions as early as possible in the operating cycle is therefore important passive components. Enhanced online monitoring can help compensate for the relative lack of knowledge about degradation modes in long-lived structures and advanced materials.

Research in accurate monitoring, flaw detection and diagnosis (location and size), and prognostics for passive components is ongoing (Bond 1999; Meyer et al. 2012a), although no PHM systems for passive components have been deployed in operating NPPs to date. The technical gaps in available ISI and NDE methods were identified in Bond et al. (2009a) and Bond et al. (2009b). Some emerging NDE techniques may be applied for continuous online monitoring of components susceptible to SCC (Cumblidge et al. 2009; Bond 2010; Doctor et al. 2010; Bond et al. 2011a; Bond and Meyer 2011; Bond et al. 2011b). The combination of new (and possibly currently unknown) degradation mechanisms and the increase in the number of components that become susceptible to aging-related degradation as plants transition to longterm operations are likely to challenge the capabilities of available ISI technology (Doctor 1988; Dobmann 2006; Bond et al. 2009c). Current nondestructive evaluation techniques used for ISI are typically applied to detect large flaws that occur near the end of component life. In order to manage aging, recent years have seen a move towards NDE for early damage detection in NPP materials (Fantoni et al. 2009; Bond et al. 2011a). There is a possibility that such early physical damage can be detected as the change of locally averaged material properties with appropriate sensors. An assessment that relates technology to various phases in degradation development for PMMD was recently prepared (Bond et al. 2011a). There is also growing interest in sensors and technology for on-line monitoring for the detection of early damage in structural materials (Inman et al. 2005; Bond et al. 2008c). Monitoring for early detection of materials degradation requires novel sensors and enhanced data integration techniques. A range of acoustic and electromagnetic measurement methods may be suitable, including nonlinear acoustics (Matlack et al. 2012), eddy current (Raj et al. 2003; Lois and Ruch 2006), and magnetic Barkhausen emission (Raj et al. 2003; Dobmann 2006). However, there are still no accepted measurement technologies for the detection and assessment of some degradation mechanisms unique to NPPs, such as void swelling. A recent review of monitoring techniques for passive systems is given in Meyer et al. (2011a). Several key areas of effort have been identified for passive SSCs, including the reactor pressure vessel and Class 1 metal components, reactor vessel internals, concrete structures, cables, buried piping, and large transformers. These are summarized below.

\subsubsection{Metals}

Currently, degradation in the reactor pressure vessel (RPV) and Class 1 components is managed through periodic ISI as mandated by the ASME BPV Code, with risk-based principles used to determine ISI intervals and the components for inspection in any given interval. In Class 1 components, issues of concern with respect to long-term operation include (Griffith et al. 2012): 
- stress corrosion cracking;

- helium-induced degradation and cracking in weld repairs;

- phase transformations due to irradiation;

- crack initiation, especially in nickel-based alloys;

- embrittlement and hardening of RPV steels.

Other degradation mechanisms also become important when considering advanced reactor designs (O'Donnell et al. 2008), including high-temperature effects on new materials such as 9Cr-1 Mo steel. Each of these degradation types, as well as other degradation mechanisms that occur in these components, likely have different underlying mechanisms (many of which are poorly understood) that drive the accumulation of damage and initiation of cracking. The components in which materials susceptible to these mechanisms are used also differ in scale from small-bore piping to the RPV. These two issues combine to create significant challenges in the development of NDE methods for detecting degradation in metal components.

Recent research has identified several novel techniques that could be applied to monitoring metallic components for degradation mechanisms of concern (Raj et al. 2003; Schuster et al. 2004; Meyer et al. 2011b). These techniques include:

- Guided ultrasonic waves (Meyer et al. 2012b),

- Magnetic and electromagnetic methods including eddy current testing, magnetic Barkhausen noise (Raj et al. 2003), etc.

- Advanced ultrasonic measurements, including nonlinear methods (Matlack et al. 2012), diffuse field methods (Ghoshal and Turner 2009), etc.

- Other techniques such as potential drop methods, Seebeck measurements, etc.

As stated earlier, acoustic emission (AE) is currently the only approved monitoring technique that is listed in the ASME BPV Code. A number of studies on the use of AE for monitoring nuclear components have been performed, and applications include crack growth monitoring, leak detection, and loose part detection and monitoring (Harris and Dunegan 1974; Hutton et al. 1984; Hutton 1993; Hutton et al. 1993; IAEA 2008b; Ai et al. 2010; Meyer et al. 2011b). AE was also deployed for a limited field trial to monitor the growth of cracks in two RPV nozzles at Limerick Generating Station Unit 1 (BWR) reactor and the Watts Bar Unit 1 (PWR) reactor (Hutton et al. 1991; Hutton et al. 1993). The flaws were known to exist and had first been characterized using conventional ultrasonic inspections. These tests demonstrated the ability of AE testing to discriminate signals due to crack growth from background noise caused by an active coolant loop and the ability to perform monitoring on components at temperatures near $300^{\circ} \mathrm{C}$ (achieved through the use of acoustic waveguides to protect the transducers from the high temperature components). AE was deployed for the duration of two fuel cycles at Limerick Unit 1 and operated well for the first fuel cycle. The equipment experienced degradation during the second fuel cycle as a result of thermal exposure. 


\subsubsection{Reactor Vessel Internals}

Reactor internals consist of components that maintain the structural integrity of the reactor core, and include components such as the core shroud, baffle-former bolts, guide tubes, jet pumps, etc. Though internal to the core, components such as fuel assemblies, control rods, and in-core monitors are not considered reactor core internals. Reactor internals are exposed to environmental stressors, such as temperature, irradiation, local $\mathrm{pH}$ and acid concentration, coolant flow, applied loads, etc. PWR and BWR designs entail different core internals; even different PWR vendor designs are sufficiently different to have different core internal systems. In all cases, the majority of reactor internal components are made from stainless steels due to their corrosion resistance, toughness, ductility, strength, and fatigue characteristics. Studies on the effects of aging on reactor internals are summarized in Luk (1993b) and IAEA (2007) for PWRs, and Luk (1993a) and IAEA (2005) for BWRs.

Reactor internals are not components of the reactor primary containment system and are not considered safety-critical. In most cases, internals can be replaced if necessary and their failure will not weaken the system itself. However, failure of these internals can create conditions that challenge the integrity of the primary containment system, and can cause expensive unplanned outages for repair. Currently, reactor core internal degradation is managed through visual inspection of accessible parts, on an approximately 10 -year cycle. There is both a safety and an economic benefit to investigating techniques for degradation assessment of reactor internals that enables reliable long-term operation.

Known degradation modes are shared for PWR and BWR components; these include corrosion, stress corrosion cracking (SCC), irradiation-assisted SCC (IASCC), fatigue, embrittlement (both thermal- and radiation-induced), and creep and stress relaxation. Many of the known degradation modes are agerelated. Mechanisms of particular concern for long-term operation include:

- IASCC of baffle-former bolts,

- Fretting wear of control rod guide tubes,

- Swelling and void formation.

Currently, the ASME BPV Code mandates periodic visual inspection of reactor vessel internals and removable core support structures. The inspection is performed using industry guidelines documented by the BWR Vessel and Internals Program (BWRVIP) and the Materials Reliability Program (MRP), which provide additional guidance regarding the inspection requirements and acceptance criteria. Visual inspection can detect distortion, cracking, loose or missing parts, wear, or corrosion. Current guidelines also require the use of alternative confirmatory inspection methods (such as ultrasonics or eddy current inspection) on select components (EPRI 2008c). Ultrasonic examination is useful for inspecting critical reactor vessel components, such as baffle bolts and guide tube support pins. Remote visual inspections of reactor internals can detect surface flaws, but the method has many shortcomings. Only components which are accessible can be visually inspected; components in inaccessible regions, those with surface deposits, or with complicated geometry cannot be effectively inspected through simple visual examination. The technique is also subject to variability due to lighting and the condition of the surface (shiny, dull finish, or oxide layer) (Cumblidge et al. 2004; Cumblidge et al. 2007). Additionally, visual inspection cannot detect subsurface faults or partial through-wall cracks originating on the hidden surface. These techniques are currently not used for online monitoring of component degradation; instead these are used for periodic in-service inspection. Several on-line monitoring techniques are recommended to 
give indicators of internal conditions during use, including loose parts monitoring, neutron noise monitoring, direct vibration monitoring, and primary water chemistry monitoring. These on-line techniques do not directly detect degradation of core internals, but they may provide relevant information during plant operation, when visual inspection is not feasible.

In advanced reactors, structural and safety-related components made of non-metallic materials such as graphite are also being considered (Kunerth and McJunkin 2011). These materials bring additional challenges in terms of inspection and monitoring, as the mechanisms of interest (such as void-swelling and loss of structural strength) may not be directly measurable using remote visual methods. Alternative inspection methods, including ultrasonics and acousto-ultrasonics, eddy current inspection, and radiography have been proposed for these materials (Kunerth and McJunkin 2011).

\subsubsection{Concrete}

In NPPs, two major concrete structures exist: the reactor containment and the nuclear island. These two structures have unique features and challenges. Concrete is exposed to the external environment (i.e., heat, humidity, etc.) as well as irradiation, the combined effects of which, over long-term exposure, may impact structural strength, integrity, and shielding effectiveness. Currently, degradation in concrete components is detected through periodic inspection as mandated by the ASME BPV Code. The reactor containment presents a major challenge for any type of inspection due to its large size; inspection of the entire containment is expensive in terms of both time and cost. Development of intelligent monitoring techniques can help focus the more intensive ISI to areas of the structure at particular risk for faults. The nuclear island is built of rebar-enforced concrete, which likely requires different inspection methods, and access is limited.

In general, concrete structures in NPP have a history of high reliability. However, several cases of degradation are known from operating experience, and include cracking, spalling, corrosion of rebars, water infiltration, etc. Other known degradation modes in concrete (in NPPs and elsewhere) include chloride attack, alkali-silica reactions, sulfate attack, carbonation, freeze-thaw, dry-out, shrinkage, creep, thermal fatigue, aggregate growth, decomposition of water, and leaching of calcium (Naus 2009). In many cases, deterioration can be accelerated by the simultaneous occurrence of multiple degradation mechanisms. Experience with concrete structures in nuclear environments indicates that prolonged exposure to irradiation and/or high temperature can result in decrease in tensile and compressive strength which can compromise the performance of the concrete structure, and potentially degrade shielding effectiveness (Shah and Hookham 1998; Norris et al. 1999; Naus 2009). The properties of concrete depend on the behavior of the coarse aggregate material used in the mix, resulting in differing properties due to differences in concrete composition, which further complicates inspection and degradation detection. The combination of large scale structures with possibly limited access and differences in composition creates significant challenges in the development of NDE methods for detecting degradation in NPP concrete.

Currently, degradation assessment of concrete structures in NPPs is performed through visual inspection. The examination of concrete containments is covered in article IWL-2000 of Section XI of the ASME Code. Components covered within the scope of this article are concrete sections of the containment, metallic reinforcement systems, and post-tensioned tendon systems. Article IWL-2000 specifies two visual examination categories. The first category is referred to as a general visual 
examination. This inspection must be detailed enough to identify regions of the concrete that are deteriorating or under distress in order to assess the condition of structural concrete. The second type of visual examination is referred to as a detailed visual examination. The purpose of detailed visual inspections is to determine the severity of deterioration or distress at suspect sites on the concrete surface, the condition of post-tensioned tendon anchoring hardware, the condition of concrete at repair/maintenance sites, and the condition of exposed reinforcing steel. A detailed visual inspection is often triggered by the identification of suspect sites during the general visual inspection.

Inaccessible below-grade areas are subject to an environmental assessment to judge the likelihood of corrosion. This environmental assessment includes an evaluation of the below-grade conditions, existing or potential degradation mechanisms, and condition of installed protective barriers. Additionally this evaluation includes a review of design and construction criteria, in-place condition-monitoring programs, and a specification of requirements for the examination of representative samples of below-grade concrete.

Research in other industries is evaluating the effectiveness and reliability of several NDE methods for concrete inspection, particularly for concrete structures; this research may prove beneficial to developing NDE methods for monitoring concrete structures in NPPs. These techniques include (IAEA 2002; Malhotra and Carino 2004), but are not limited to:

- ultrasonic testing (including guided ultrasonic waves),

- thermographic imaging,

- radiographic imaging,

- half-cell potential and surface potential,

- polarization resistance,

- impact-echo, and

- microwave techniques.

A comprehensive review of methods for concrete inspection in NPP is presented in Naus (2007). Recent work in the area of NPP concrete inspection also includes online monitoring of post-tensioned tendons using strain gages at the Ginna plant (EPRI 2011b); this pilot study is described in a later section.

\subsubsection{Cables}

In 1993, the International Atomic Energy Agency (IAEA) initiated a coordinated research project (CRP) to address the aging management of in-containment cables at NPPs (IAEA 1997). Because it simply is not practical to inspect the over $1000 \mathrm{~km}$ of cable within an NPP, a prioritization scheme is necessary to limit cable inspection programs to a manageable level. In the context of aging management, the outer jacket and electrical insulation (both made of polymeric materials) are typically considered most significant. Typical cable architecture consists of one or several conductors individually wrapped with electrical insulation and bundled inside of a protective jacket (IAEA 2000). Three types of cables are of concern in nuclear power plants: power, control, and instrumentation cables. Degradation of these cables has largely been ignored because they are considered to be passive, long-lived components with high historical reliability. However, longer service life entails increased exposure to environmental stressors, 
such as temperature, irradiation, moisture and humidity, local oxygen concentration, vibration, flooding of underground cables, etc. Under these conditions, the material of most concern appears to be the polymeric insulation and jacket material on these cables (IAEA 2000). Longer cable circuits may pass through several different environments over their length routed through the plant. Licensee data has shown that the number of cable failures is already increasing with plant age, even within the rated 40-year lifetime (Villaran and Lofaro 2010).

Cable integrity and function can be monitored indirectly through in-service tests of safety-related systems and components. Cable monitoring programs are summarized in NUREG/CR-7000 (Villaran and Lofaro 2010). However, adequate function of the cables under test conditions does not indicate the same performance during high stress events, such as operation when fully loaded or during extended periods as under normal service operation or design basis events. Several laboratory tests are available for cable monitoring; however, these require that a sample of the cable aged under the same conditions as the location of interest is available for testing. These tests are generally undesirable for one of two reasons: (a) the test may be destructive, meaning that the number of cable samples limits the frequency of the test, or (b) cable samples may not have experienced the same environmental stressors seen in inaccessible locations. A number of in situ cable monitoring techniques are currently employed, including visual inspection, the compressive modulus test, dielectric loss test, insulation resistance and polarization index, AC voltage withstand test, partial discharge test, DC high potential test, step voltage test, infrared thermography, etc. These tests all suffer serious disadvantages. Many are only applicable to accessible lengths of cable, such as visual inspection and the compressive (or indenter) modulus test. Anandakumaran (2007) correlated the results of elongation at break (a destructive test) to those of the compressive modulus (a nondestructive, in situ test). A threshold for the compressive modulus was determined beyond which successful performance during LOCA scenarios could not be guaranteed (indicated by $50 \%$ elongation at break). However, the compressive modulus is a manual test which can only be performed periodically on accessible lengths of cable. Some techniques require the cable to be disconnected at one or both ends, including the dielectric loss test, insulation resistance and polarization index, AC voltage withstand, DC high potential, and step voltage tests. Additionally, several of the electrical tests involve high voltage, which can damage the insulation during testing (i.e., AC voltage withstand, partial discharge, and DC high potential tests). Two techniques have been developed and demonstrated for non-destructive, in situ cable inspection: time domain reflectometry (TDR) and line resonance analysis (LIRA).

TDR operates on the same principles as radar (IEC 2010). A pulse of energy is transmitted down a cable from one end, which must be disconnected from the system for testing, and is reflected back when it encounters any change in electrical impedance along the cable. These changes may be due to the far end of the cable or faults and degradation along the length of the cable. The time necessary for the pulse to travel to the impedance change and reflect back to the open end of the cable can be used to determine the location of the impedance change. These measurements can identify the location and magnitude of an impedance change due to electrical or insulation faults or the presence and location of water; however, this requires accurate baseline data for comparisons. When appropriately applied, TDR is able to identify changes in cable impedance due to loose connections, moisture, and cracks in cable conductors, connectors, and insulation. Transient conditions, such as immersion or vibration-induced failure, will only be detected if they are present during testing. TDR is applicable to low- and medium-voltage cables, as well as cable insulation and jackets of all types. The pulse used to detect faults is very low power and non-destructive to the cables being investigated. 
LIRA analyzes electrical test signals from a waveform generator by modeling the cable's wire system using transmission line theory (Fantoni and Toman 2006; Fantoni et al. 2009; Fantoni 2010). Through narrow-band frequency domain analysis of high frequency resonance effects, LIRA can be used to detect local or global changes in the electrical properties of a cable, which may result from insulation faults or degradation. The cumulative phase shift of the input impedance gives a condition indicator for aging and small defects, while amplitude changes account for larger effects. Because LIRA tests can detect small changes in the electrical properties in insulation materials, localized and bulk thermal aging can be detected and identified well before significant physical deterioration occurs. LIRA was developed at Halden Reactor Project from 2003-2005, and then further developed by Wirescan AS. LIRA has been tested extensively with low, medium, and high voltage cables, both in laboratory tests and in-situ experiments. Work with EPRI from 2006-2007 tested the capabilities of LIRA to identify cuts, gouges, and thermal aging in cables. Unlike TDR, LIRA can be performed in situ without disconnecting the cable. LIRA can be performed online by accounting for the effects of loads attached to the cable during the results analysis.

TDR is currently widely used in NPPs to assess instrumentation cable condition; however, this technique cannot be applied during plant operation because the cable must be disconnected at one end. It detects local, significant anomalies but is not as effective for minor flaws which may be precursors to larger faults. Additionally, the method requires accurate baselines for comparison to determine the location and magnitude of a fault. LIRA overcomes many of the disadvantages of TDR - it can be performed on-line, and it can detect small changes in electrical parameters. However, research in LIRA is ongoing. Currently, the test is not simple to perform nor the results to interpret; execution and analysis will need to be largely automated for wide-scale application in NPPs.

In June 2010, the USNRC issued a draft regulatory guide on condition monitoring of cables (NRC 2010a), which states that no single, nonintrusive method is currently available for accurately predicting the survivability of cables under accident conditions. Therefore, a combination of techniques may be necessary to give a robust view of cable condition. Recent work is focused on evaluating the available cable-testing techniques and developing a holistic approach to integrating the results of multiple testing modalities (Hashemian and Bean 2011). This integration is expected to improve the range of faults and degradations that can be detected and the robustness and confidence of those detections.

\subsubsection{Buried Piping}

Buried piping is in direct contact with soil or concrete. Three types of buried piping should be considered: safety-related pipes, non-safety-related pipes which convey radiologically contaminated fluids, and other non-safety-related pipes conveying water, fuels, oils, gases, etc. Potential impacts of buried pipe faults and failures include:

- Safety and operational challenges,

- Negative environmental impacts,

- Additional regulatory requirements,

- EPA violations with adverse industry publicity, 
- License renewal delays, and

- Heightened opposition to extension of existing license and new plant construction.

Buried piping is subject to external environments (chemical, geotechnical, and civil-structural) that may be unique to each installation or site. A number of materials (mostly metallic, although concrete is used for large-diameter piping such as water-intake piping) are used for buried piping in operational NPP (Braverman et al. 2005). In recent years, there is increased interest in the use of large-diameter HDPE piping for some non-safety related buried piping (SECY-09-0174 2009). Buried pipes also come in a large range of sizes, varying from over $10 \mathrm{ft}$ diameter down to 2 inches and smaller. These issues together provide significant challenges to inspecting large installations of buried pipes. Recently, the nuclear industry began an initiative through NEI to develop guidelines for the management of buried piping integrity (NEI 2010). Additionally, research at EPRI focuses on programs to control the degradation of buried piping (EPRI 2008b).

The main causes of degradation in buried piping include fouling or debris, soil movement, surface traffic, and corrosion (Braverman et al. 2005; NRC 2005a, b). Buried piping is susceptible to degradation (corrosion and fouling) on both the outer diameter (OD) and inner diameter (ID) surfaces. Preventative measures are taken for metallic buried piping which may corrode on the OD, such as coating with corrosion resistant materials or applying cathodic protection systems. While buried piping is normally reliable, damage to the coatings can lead to localized corrosion and small leaks. Underground piping at some nuclear power plants has degraded to the point that through-wall leakage has occurred, leading to, in a few cases, releases of tritium into surrounding groundwater (GAO 2011). The plants' safety systems continue to function properly despite these leaks. EPRI's recommendations for monitoring and controlling the degradation of buried piping are given in EPRI (2008b).

Buried piping at NPPs offers a unique challenge because these pipelines were not designed in a way that readily accommodates inspection techniques employed in other industries, such as pigging. NPP piping may have more bends than can be accommodated with these techniques and may lack the necessary access points. Therefore, inner diameter inspections are prohibitive for long stretches of pipeline that consists of elbows, tees, or other sources of sharp redirection. Additionally, access to buried piping outer diameter is restricted due to contact with soil or concrete.

Currently, most inspection of buried pipes involves excavating to visually inspect sections of the pipe. Alternative technologies that may be relevant include remote visual systems, ultrasonic inspection methods for piping wall thickness evaluation, and electromagnetic inspection methods (such as eddy current) for detecting areas of corrosion (Braverman et al. 2005). In-line inspection using "smart pigs" is a widely applied technique for piping inspection in the petrochemical industry; its applicability to NPP buried piping is under evaluation (Kirby 2012). Guided ultrasonic wave (GUW) is considered one of the leading technologies for performing inspections of buried piping (Ahmad et al. 2009) but is limited to inspections of a few 10's of meters and also encounters difficulties with inspecting pipeline beyond elbows, tees, or other joints. Thus, full-length inspections of buried pipes with GUW would require excavation every 20-30 meters or beyond every elbow for placement of a GUW probe. NDE technologies employed by the oil and gas industries for inspecting buried piping are not immediately transferrable to NPP buried piping for the reasons enumerated above.

Several other entry and excavation tests, including hydrotests, are being explored by the industry. Additional inspection methods that may be applicable include liquid penetrant testing, magnetic particle 
testing, radiography, pulsed eddy current, hydrotest, remote field electromagnetic inspection (Lord et al. 1988), and corrosion tests such as electrical resistance probes, corrosion coupons, and linear polarization resistance. Many of these methods require physical access to the section of pipe under test. The use of remote probes that can be inserted into the piping system for in-line inspection is also of interest. However, questions remain regarding access to NPP piping systems that were not designed with inspection in mind.

\subsubsection{Transformers}

The reliability of electric transmission grids depends on the availability of high-voltage transformers. Although transformers have been historically over-designed, economic competition in recent decades has led to lower-cost, lower-reliability transformers in service. This implies that transformer degradation is a concern for transformers of all ages: over-designed transformers installed in the 1970s, 1960s, or earlier are nearing the end of their original design life, and transformers installed after approximately the mid1980s are also experiencing wear-out failures due to the lower reliability standard. In fact, transformers in service in the USA are experiencing an exponential increase in explosions and failure rate (Bartley 2002). Replacing existing transformers is costly both in terms of time and money; replacements can cost between \$3- and \$6-million with lead times of more than a year. However, unanticipated failures can affect generating stations, resulting in increased maintenance costs and lost revenue. In fact, a recent transformer failure at the Indian Point Unit 2 reactor caused a reactor trip and resulted in the reactor being offline for over two weeks. Many groups are investigating the use of existing technologies to better monitor the condition of high-voltage oil-filled transformers. These techniques can be coupled with advanced fault detection, diagnostic, and prognostic methodologies to provide a full health-monitoring system for transformers.

Bartley (2003) identifies twelve failure modes which were experienced by members of an international delegation of the International Association of Engineering Insurers. Of these twelve failure modes, two of are particular interest because of their relative ubiquity in legacy transformers: insulation failure and oil contamination.

Traditional transformer monitoring techniques have seen widespread use for many years or even decades. Other, more advanced technologies are still in the research phase, but may be applicable for integration into a full health monitoring system. These methodologies include: dissolved gas analysis, insulating oil quality testing, moisture content measurements, various methods of power factor testing, winding resistance and ratio testing, thermography, recovery voltage testing, winding insulating oil monitoring, tap changer monitoring, internal temperature measurement, and others (Lapworth et al. 1995; Wang et al. 2002). Several different transformer monitoring techniques are currently being used to measure degradation of transformers. One of the most common and widespread technologies is the measurement of dissolved gases within the transformer (Heathcote 1998). Moisture content, another degradation parameter, can often be measured with the same equipment used to perform dissolved gas analysis (DGA) (USDI 2003). Oil quality tests can often discover the chemical breakdown of the oil (USDI 2000, 2003), but are less useful than DGA and moisture content measurements because failure occurs more frequently as a result of dissolved gases and moisture. Ultra-high frequency (UHF) monitoring is used to measure partial discharge events in transformers (Judd et al. 2004; Catterson et al. 2008). Finally, several tests exist to quantify insulation degradation (USDI 2000). 
Partial discharge (PD) is an electric discharge that only partially bridges the gap between conductors. Such discharges can indicate a loss of dielectric strength in the insulation or an increase in electrical stress. UHF monitoring can be used to isolate PD arcing. UHF sensors may be mounted external to the transformer through dielectric windows, which would be straightforward to install during manufacturing and may be possible to retrofit to existing transformers in service (Judd et al. 2004). Researchers at the University of Strathclyde have worked to incorporate UHF results in an automated monitoring and fault detection system. Their work focuses on the use of Multi-Agent Systems (MAS) for condition monitoring (McArthur et al. 2004). The system is modular, with separate agents for each task, including: data handling agents, isolation agent, classification agents, corroboration agent, engineer assistant agent, etc. The modular nature of the system makes it particularly attractive for combining with agents which perform fault detection and diagnostics based on other information, such as DGA or SCADA results (Catterson et al. 2005). The developed on-line monitoring system was deployed on two transformers nearing end of life (Catterson et al. 2009). This system used data collected from 50+ sensors to detect and diagnose anomalies. The MAS system was shown to be flexible and extensible to incorporate output from other monitoring and interpretation techniques.

In addition to detecting PD, UHF sensors can be used for active interrogation of the mechanical structure to detect displacement. After a potentially damaging event, such as through faults, harsh loading, or physical deformation, the same UHF sensors installed for PD monitoring could be used to determine if the internal structure of the transformer has been permanently altered or has returned to its original state (Judd et al. 2004).

A survey of the literature describing available and developing transformer monitoring techniques indicates that it may be possible to perform prognostics using data that can be collected with existing transformer monitoring technologies. Initial investigations in using existing data sources for prognostics are reported in Strong et al. (2011) and Coble et al. (2011).

\subsection{Fielded Systems and Systems Currently in Development}

While significant work still exists in many areas of condition monitoring and PHM before it can be applied on a large scale in NPPs, several utilities, companies, and other groups have made real progress in deploying condition monitoring and health assessment systems on specific subsystems. In addition to the sensor calibration monitoring system deployed at Sizewell B described earlier, several efforts that move toward PHM are described below.

\subsubsection{AREVA}

AREVA has worked with health assessment systems since the early 1980s with the Fatigue Monitoring System (FAMOS). FAMOS was developed in response to a requirement by German licensing authorities to better understand actual component loadings during plant operation. Initial recordings of thermal loadings indicated that operating conditions of the plant differed from the assumptions and predictions made during plant design. This discovery led to widespread implementation of FAMOS in German NPPs and other plants, with deployment in more than 20 NPPs since 1988. Additionally, FAMOS is anticipated to be used in the new European Pressurized Water Reactor (EPR) (Pöckl and Kleinöder 2007). FAMOS uses operational loads continuously measured onsite with a simplified stress-based fatigue (SSBF) estimation method to evaluate the actual fatigue experienced by 
key SSCs. This analysis is performed after every operational cycle; the results of this fatigue monitoring and estimation can provide the basis for optimizing operational modes and future fatigue usage (Poeckl et al. 2001).

In addition to fatigue monitoring and estimation suites, AREVA is deploying enabling technologies for PHM, including digital I\&C systems and OLM suites, in several existing and new reactors. In 1984, AREVA deployed a digitized monitoring and control system for the power and protection control system for the reactor core at the Paluel plant. In 2011, EDF contracted with AREVA to upgrade the monitoring and control safety systems for 20 reactors at 8 plants (AREVA 2011). AREVA is also deploying integrated OLM in new, Generation III+ LWRs, such as the new reactors at Olkiluoto in Finland, Flamanville 3 in France, and Taishan $1 \& 2$ in China, and the proposed reactors at Hinkley Point $\mathrm{C}$ and Wylfa in the U.K. (AREVA 2012).

\subsubsection{Ginna Containment Monitoring}

A pilot study of online monitoring of the reactor containment vessel (RCV) at an operating NPP was undertaken by EPRI at the Ginna plant. Currently, fourteen randomly selected containment tendons are tested once every five years by measuring lift-off loads. These tests are expensive (costs of $\sim \$ 500 \mathrm{~K} /$ test), are manually performed, and have implications for worker safety. Three monitoring techniques were employed at the Ginna plant to monitor post-tensioning tendons: tendon load monitoring through fiber optic gages, rebar and concrete strain monitoring, and concrete surface strain monitoring with digital image correlation (DIC) (Lindberg 2011). Fiber optic strain gages were employed to measure tendon strain in the RCV by EPRI's NDE group (EPRI 2011b). The RCV wall is made of reinforced concrete with 160 post-tensioned, ungrouted tendons. The Ginna RCV has some features not employed at most plants; for example, vertical, prestressed tendons connected to rock anchors in the underlying bedrock (Shah and Hookham 1998). Fiber optic strain gages were installed on the shims of 20 tendons to collect real-time, continuous tendon force data. Data were collected at a high sampling rate $(1 \mathrm{~Hz})$ for 334 signals relating to the tendon strain for a period of 12 months. These data were used to calibrate and test an advanced pattern recognition (APR) model using 41 signals, including two temperature-compensated strain measurements for each of the 20 measured tendons and the ambient temperature. The data collection for a deployed system is expected to occur on a daily or weekly basis (i.e., one sample is collected once per day or once per week) and evaluated quarterly to detect anomalous behavior, but higher sampling rates were used for the pilot study to aid in determining the appropriate sampling rate and data analysis period. The results of applying the APR model to test data indicate good predictive performance over the range of data collected over the 12-month period; in fact, the model reliably predicted sensor values to within $0.5 \%$ error. Currently, optical fiber strain gages are suggested to augment the current periodic test, but additional testing and analysis may allow for continuous monitoring to replace periodic testing. This initial study concluded that the fiber optic strain gages can be coupled with an APR model for effective monitoring of tendon strain in the RCV.

\subsubsection{Borssele OLM System}

The Borssele Nuclear Power Station (the Netherlands), a two-loop PWR, has been in operation since 1973 and is currently licensed to operate until 2033 (NIS 2006). Borssele has provided operational data for reactor signal processing algorithm development since 1974. From 1974 through 1981, off-line signal analysis was used to monitor the condition of the plant. Beginning in 1982, real-time surveillance of 
Borssele began to monitor for changes in statistical characteristics of sensed signals. The monitoring system was used to estimate physical parameters of the reactor and in-situ testing of safety channel instrumentation using online analysis. Turkcan (1985) describes the use of noise analysis for NPP monitoring, including continuous monitoring of primary system vibrations, continuous monitoring of core physics parameters (core coolant velocity, reactivity effects, local vibration of in-core instrumentation guide tube and core support barrel), and online monitoring of noise power of plant sensors for both shortand long-term trending. This and other well-defined algorithms were used for an online, real-time monitoring system until 1997 (Turkcan et al. 1996). After a major plant upgrade in 1997, a new sensor measuring system was developed and deployed in 2001. The new plant data collection and processing system consists of two sub-systems: (1) $96 \mathrm{DC}$ signals measured at $10 \mathrm{~Hz}$ for operational history recording, deviation detection, and plant transient analysis, and (2) $32 \mathrm{AC} / \mathrm{DC}$ signals for the reactor noise diagnostic system. Core barrel motion is monitored through cross-spectrum analysis of the ex-core neutron detectors located around the reactor vessel (Barutcu et al. 2003b). Additionally, coherence function analysis of primary pump vibration signals was used to detect sensor faults, while coherence analysis of ex-core neutron detectors, primary system pressures, pressure of primary pumps (Barutcu et al. 2003a). Data from the Borssele plant have been used to develop a variety of monitoring and diagnostic routines, although these are not currently deployed at the plant. Nabeshima et al. (2003) describe a monitoring system which employs neural networks for fault detection and an expert system for fault diagnosis. In this work, a PWR simulator is used to model plant anomalies to train and test the diagnostic system. The system is also tested with data from Borssele; the expert system correctly recognized operation modes and diagnosed plant status based on the detection patterns predicted by the neural networks. Finally, Ayaz (2008) developed a system of neural networks for monitoring Borssele using so-called plant-wide and component-wide models. The plant-wide model is built using all the signals of interest, 62 signals total. Nine component-wide models are developed for different subsystems: core, generator, pressurizer, turbine, condenser, primary volume control system, steam generator feedwater system, steam generator I, and steam generator II. Twenty-three of the 62 signals are best monitored by the plant-wide model, while the remaining 39 signals are monitored by their respective component-wide (subsystem) models.

\subsubsection{Recent Industry R\&D Activities in OLM}

There is an ongoing industry program focused on developing on-line monitoring and sharing best practices (EPRI 2010, 2011a), and it is seeking to make advances in diagnostics and prognostics over the next 4-5 years. An early effort in developing prognostic capabilities looked at expanding the information contained in the predictive maintenance basis database (PMBD) to include information about possible prognostic applications (EPRI 2009a). A compendium to the PMBD highlights the applicability of prognostics and potential prognostic parameters for each class of systems and components contained in the PMBD.

Three separate, but complimentary, programs to support health monitoring of power generation systems are being developed. The Diagnostic Advisor compares actual plant conditions to the stored fault signatures in the Asset Fault Signature Database to determine if a fault condition exists and identify likely causes. Plant condition data can also be used by the Remaining Useful Life Advisor to estimate the time of failure for systems or components experiencing a fault based on several available prognostic models. Each of the main programs was developed to act either as a stand-alone product or in cooperation with the other programs. The full software suite is designed to perform fault diagnosis and 
prognosis based on available system data sources. Data sources can include plant data historians, online monitoring and fleet-wide monitoring programs, operator inspection results, etc. Additionally, these tools can serve as a repository for sharing information across the entire fleet of utilities, allowing each utility to integrate the operating history of similar plants, similar systems, and similar components. 


\subsection{Enabling Technology}

\subsection{Digital Instrumentation and Control Systems}

The nuclear industry is notoriously slow to adopt new technology because of the additional regulatory restrictions on changing the technical specifications of NPPs. Most operating U.S. NPPs still employ the 1970s era or earlier analog technology that was originally installed during commissioning. Digital upgrades have largely been incremental and applied where digital systems can replace analog systems one-to-one. The first digital I\&C system in a U.S. NPP was deployed at Oconee Nuclear Station in 2011 (Hashemian 2011b). Fully digital I\&C systems have seen more use outside of the United States. The first fully digital I\&C systems were deployed in Japan in the Kashiwazaki-Kariwa Unit 6 and Unit 7 advanced boiling-water reactors (ABWR). Mitsubishi's Tomari Units 1 and 2 PWRs also have complete digital I\&C systems. Digital I\&C systems have also been implemented in NPPs in France, Korea, Sweden, the United Kingdom, China, and other countries.

\subsection{Sensors}

Advances in sensors, including smart microsensors, ultrasonic sensors, acoustic emission sensors, radio-frequency tags, and multi-sensor modules, enable more reliable and less expensive data collection to support PHM. Sensors used for measurements inside the reactor containment must be resistant to the radiation experienced during operation; these sensors are particularly difficult to replace due to their location and the resulting exposure to personnel. Research is ongoing to develop sensors that will be robust to these harsh operating environments over longer periods of time; this work is briefly described in a later section. Currently, there is significant interest in deploying wireless sensor networks for monitoring NPP SCCs (Hashemian 2011a).

\subsubsection{Wireless Sensor Networks}

The high cost of radiation-resistant instrumentation cable makes wireless sensor networks attractive, particularly for aging systems which may require additional sensing capabilities to support OLM or replacement of aging instrumentation and cables. In fact, approximately $80 \%$ of respondents in a recent survey of I\&C personnel at NPPs indicated that they would increase CM if wireless sensor networks could be used (Kiger 2010). Several issues must be resolved before wireless sensor networks are ubiquitously adopted in NPPs for safety-related I\&C applications, including coexistence, reliability, signal propagation, and regulatory impact (Howlader et al. 2010). Many of these challenges can be addressed through thoughtful and deliberate locating of wireless transmitters and receivers. Of course, cyber security is also a significant concern when applying wireless sensors to safety-related equipment and for nonsafety-related wireless networks that potentially provide a pathway to critical attacks (Dion et al. 2010). Key issues in wireless security include interference, interception, and fading and multipath. Well-established methods for alleviating these concerns include anti-jamming, intrusion detection, and accurate channel modeling. The NRC guidelines for cyber security (10 CFR 73.54 2009; NRC 2010b) were not designed with wireless sensors in mind; however, the guidelines can be easily extended to give adequate security for wireless networks. 
Although wireless sensors and networks have not been applied to safety-related applications, wireless networks are currently used for wireless local area networks for office use, automated intelligent metering, geographical information systems, work management tools, and emissions monitoring. Voice and data communication wireless networks have been implemented on varying scales at select NPPs, including Comanche Peak, Arkansas Nuclear One, and South Texas Project, while wireless networks intended for condition, process, and environmental monitoring have been deployed at Comanche Peak, San Onofre, and River Bend.

Wireless sensor networks have been deployed in two facilities for equipment condition monitoring data transmission as part of a U.S. Department of Energy (DOE) Small Business Innovation Research (SBIR) project: Comanche Peak Nuclear Power Plant in Texas and the High Flux Isotope Reactor (HFIR) at Oak Ridge National Laboratory (ORNL) (Hashemian 2011a; Hashemian et al. 2011). The objectives of this project are two-fold: first, to evaluate and expand the technical basis for condition monitoring of active components and, second, to address challenges in deploying wireless sensor networks in NPPs. Challenges addressed include installation issues, electromagnetic and radio-frequency interference, network issues, data acquisition and qualification algorithms, analysis of wireless data, and interpretation of the results. Results of these pilot applications indicate that well-developed wireless sensor networks offer an effective method for measuring and transmitting data related to key equipment and process conditions, thereby improving the safety, reliability, and efficiency of NPPs. Concerns over interference, data acquisition, and data security can be addressed and accommodated to provide reliable, secure transmission of sensed data. Additionally, the integration of signals from existing wired sensors and new wireless sensors in existing LWRs can provide a holistic view of the health of SSCs and processes, further improving safety and performance in legacy plants.

\subsection{Monitoring the PHM Hardware and Validating Data}

Accurate detection of faults and estimation of RUL depend on accurate data from the OLM and ISI systems. Before the results of PHM algorithms are used for decision making and O\&M planning, the data fed into these systems must be validated. Much of the technology needed to monitor the hardware (sensors, cables, etc.) and data for the PHM system has already been developed. Existing sensor calibration monitoring methods can be used to validate the sensed data needed for PHM systems. Existing and developing cable monitoring methods can be employed to ensure that data transfer has not been compromised. Additional data validation routines, such as data reconciliation, can be employed to increase confidence in measurements used for health monitoring. It is important to leverage existing technology developed to monitor the physical system (i.e., NPP) to also monitor the monitoring system. Uncertainty in the measurements and data used by the PHM algorithms will inflate the uncertainty in the results of each module - monitoring and detection, diagnostics, and prognostics. By monitoring hardware and validating PHM data, this uncertainty can be managed, improving the confidence in PHM results and in using these results for decision making and O\&M planning.

\subsection{Prognostic Architectures (Software)}

A recent review of commercially available software products for supporting PHM systems (Lybeck et al. 2011) found a number of integration architectures currently available for deployment of PHM.

Development of each of the modules in a full PHM system for deployment in an NPP is both costly and time-consuming. An existing software framework can be leveraged to demonstrate the use of PHM in an 
existing NPP with reduced development time and cost. Thirteen products identified through literature and internet searches were evaluated based on a consistent set of criteria: open, modular architecture; platform independence; graphical user interface for system development and/or results viewing; web-enabled tools; scalability; and standards compatibility. The thirteen products were classified into four rough groups of software: research tools, PHM system development tools, deployable architectures, and peripheral tools. Eight software tools fell into the deployable architectures category. Of those eight, only two employ all six modules of a full PHM system. Five systems did not offer prognostic estimates, and it is unclear how easy it would be to incorporate third-party prognostic algorithms. Both OSyS and Impact Technologies provide a pair of tools that can fulfill all six modules when used in tandem: AOC and EHM, and SignalPro and ReasonPro, respectively. Depending on the purpose of the applied system, each of these products has its own advantages and disadvantages. Complete details of the software review can be found in the original report. 



\subsection{Codes and Standards}

The Nuclear Energy Standards Coordination Collaborative (NESCC) reviews the codes and standards relevant to nuclear energy applications (ANSI). Codes and standards covering several topics are of particular interest to PHM in NPPs. The NRC only accepts NDE methods endorsed by the ASME Boiler and Pressure Vessel Code for monitoring large passive structures in NPPs. This Code currently only endorses AE for monitoring passive components, and even then only under specific conditions. The Code will need to be continually updated to reflect the current state of the art in NDE methods as it progresses.

Additionally, a large number of disparate standards exist which pertain to prognostics and PHM systems. Sheppard, Kaufman, and Wilmering (2008) review the relevant standards in IEEE and other organizations related to prognostics and highlight the importance of predicting RUL as a key feature of prognostics; however, they propose no single, unifying standard. An effort is currently underway to develop an IEEE standard for prognostics, led by researchers at the Center for Advanced Life-Cycle Engineering (CALCE) at the University of Maryland; however, the timeline and intended content for this standard is currently unclear.

ISO 13374 is a collection of standards that define a general condition monitoring architecture (framework) for machines. Part 1 (ISO 2003) focuses on general procedures; Part 2 (ISO 2007) focuses on data processing; and Part 3 (ISO 2012) covers communication. MIMOSA OSA-CBM is an implementation of the ISO-13374 functional specification (Walter 2006). OSA-CBM uses the Unified Modeling Language (UML) to define the standard, separating the information from the technical interfaces used to exchange or communicate the information. This implementation allows vendors and integrators to implement the most appropriate technologies for their application. ISO 13381 (ISO 2004) provides general guidelines for the development of machinery prognostics, addressing terminology, concepts, uncertainty, and degradation modeling (Tobon-Mejia et al. 2010). ISO 18435 (ISO 2009) describes an integration model and interfaces to facilitate integration of CBM related information with operating and environmental information to support optimal decision-making for effective and efficient manufacturing (Carnahan et al. 2005).

The development of a standard covering PHM for electronic systems was recently approved by the Institute of Electrical and Electronics Engineers (IEEE). While the proposed standard focuses on electronic systems, it can provide a framework for developing a general standard for PHM for any complex engineering system, as well as specific standards for active and passive nuclear SSCs (IEEE).

Standards for condition monitoring, prognostics, PHM, and NDE techniques exist, but these are largely not specific to the nuclear industry and have not been reviewed by the NRC for application to NPPs. Work is needed to develop nuclear-applicable standards for advanced NDE and PHM techniques and to have these standards reviewed and endorsed by the NRC. 



\subsection{Research Needs and Opportunities to Support PHM in NPPs}

Prognostics and health management is a key need for improving the safety and economy of nuclear power generation moving forward with both LTO and new builds. Several general areas of further development needs exist to bring PHM from the research arena to deployment in NPPs. Some of these needs are specific to the application to NPPs and nuclear components, including high-fidelity physics-offailure models, experimental failure and aging data, and optimal sensor placement (particularly for passive components) to give adequate coverage at reasonable cost. Some areas of research are more general to the PHM community as a whole (uncertainty analysis and propagation, online performance metrics, verification and validation of PHM algorithms and models, etc.), and ongoing research in other fields can likely be leveraged to address these needs for NPPs. Finally, well-defined methods for incorporating the results of PHM algorithms into a more holistic view of plant operation, maintenance, and decision making are needed to provide a practical advantage for the use of PHM in terms of safety and economics.

\subsection{Exemplar Data and Models}

Individual prognostic algorithms require a population of exemplar degradation paths for each fault mode of interest for model training and validation. These exemplar degradation paths can be sensed data, collected in situ or through well planned experiments, or simulated with high fidelity physics of failure models. Aging, degradation, and failure data are rare for safety-critical and high value systems, such as NPPs, because these systems are rarely allowed to run to failure once degradation has been detected. Development of high-fidelity physics of failure models is expensive and time consuming. The underlying physics of some failure modes, such as SCC, remain too poorly understood for development of these models. Realistically, both experimental data and physics models will be needed to fully describe the variety of failure modes of interest in active and passive SSCs.

\subsection{PHM for Transient Operation}

Many proposed PHM algorithms presume that operation is (at least nominally) steady-state to support application of statistical analysis techniques. Of the techniques that can be applied to non-stationary signals, none were developed specifically for transient operation. However, additional information about equipment health can be gleaned from transient operations, such as during start-up or power ramping, when stressors are often much greater than those seen during steady-state operation; these increased stressors may provide earlier, more accurate indications of incipient faults. Initial research into transient prognostics is ongoing (EPRI 2011d). Two exploratory projects investigating transient prognostics have been initiated at the University of Tennessee (as of 2012). The first project investigated transient prognostics for small transfer pumps (Sharp et al. 2010); the second investigates motor degradation (Sharp et al. 2011, 2012). For both of these systems, there are clearly additional stresses seen by the equipment during start-up, and statistical analysis methods are being explored and developed to extract information from the non-stationary signals collected during these transients (Strong and Hines 2012). The statistical analysis methods developed for transient prognostics may be of particular interest for SMRs, which will likely be load-following and will experience more operational transients than base load NPPs. 


\subsection{Sensor Design and Placement}

Novel methods for ISI of passive components are needed, particularly to support online condition monitoring of Class 1 and in-vessel components, to provide more effective inspection of complex geometries and inaccessible components. In particular, sensors that can withstand the harsh environment inside the pressure vessel are needed, although there is ongoing research in this area in the case of piezoelectric ultrasonic sensors (Parks and Tittmann 2011). Currently used remote visual cameras are radiation-hardened but are still incapable of surviving for more than a few hours in the radiation environment. There is also a need to develop methods to transfer measurements from inside containment to the outside without compromising structural integrity.

Another challenge to applying PHM to NPPs is sensor placement, particularly for passive components. While active components can, in many cases, be monitored using the measurands currently collected for the existing I\&C systems, some additional instrumentation (such as permanent vibration monitors) would improve model accuracy and robustness. Research in optimal sensor placement for fault observability and reliability of active components has looked at application to the IRIS reactor (Li and Upadhyaya 2011; Upadhyaya and Li 2011), though the methodologies employed are more widely applicable. The algorithms used in this work could easily be extended to incorporate the information already available from the plant I\&C system, further minimizing the cost associated with deploying additional sensors specifically for active component health monitoring. Additionally, legacy systems do not have the instrumentation required for continuous, online prognostics of passive components. The proposed NDE methods for monitoring passive components offer limited coverage for each scan; multiple measurements at different locations (and perhaps multiple types of measurements) will be necessary to provide coverage of an entire passive structure. Due to cost concerns of deploying many sensors (either through retrofitting legacy reactors or embedded in new plant designs), it may not be feasible to provide $100 \%$ coverage of every important passive structure. Risk analyses such as FMECA may be used to determine the components and locations of most interest in monitoring for degradation in order to reduce the monitoring burden. Research in sensor placement for detecting sub-surface cracks in aircraft structures found that the selection of frequency and placement could only be determined with a good understanding of the failure mechanisms and application problem (Teo et al. 2009); the research in physics of failure models described above will be necessary for accurate sensor placement, particularly for passive components.

\subsection{Uncertainty Quantification and Propagation}

Additional research needs are generic to the development and deployment of PHM systems. The research in quantifying prognostic uncertainty was described above, but this field is still evolving. In addition to understanding and evaluating uncertainty in RUL estimates, we need to understand how uncertainty in each module of a PHM system propagates to later modules and how to control this uncertainty. For example, uncertainty in the data collected from a system will contribute to uncertainty in the fault detection, the diagnosis, and the eventual prognosis. The uncertainty calculated at each stage needs to account for the uncertainty in the stage(s) before it. Additionally, the uncertainties for specific components will propagate through to subsystems, systems, and the plant as a whole. Some of this may be addressed by prognostic-informed PRA analysis, which will be described later. 


\subsection{Online Prognostic Model Performance Assessment}

Uncertainty quantification can give some indication of prognostic performance metrics, but additional measures are needed to characterize the performance of a prognostic model online. Online performance measures for monitoring, fault detection, and fault diagnostics are well established and understood (Hines et al. 2008a), but performance metrics for prognostics have largely focused on offline analysis of algorithms as opposed to online analysis of a particular implementation (as described in Section 4.4.2). Many of these metrics rely on the actual failure time as ground truth data for comparison; obviously this information is not available during online prognosis. Methods to evaluate the accuracy, robustness, and confidence level of RUL predictions as they are made will be necessary to determine if the results provide actionable information for O\&M planning.

\subsection{Verification and Validation}

Many prognostic algorithms have been developed and tentatively applied to a variety of systems with varying levels of success, but there currently is little attention paid to verification and validation (V\&V) of prognostic algorithms. In order to apply prognostics to NPPs, a rigorous V\&V method must be developed and approved by the NRC. Some research has begun to address V\&V for prognostic algorithms, though none specific to the nuclear industry; a review of $\mathrm{V} \& \mathrm{~V}$ research to date is given by Liu et al. (2011). A web-based V\&V tool was developed specifically targeted at the JSF, which provided $\mathrm{V} \& \mathrm{~V}$ metrics for detection, diagnostics, and prognostics (Orsagh et al. 2005). Some of the verification methods used by meteorologists, climatologists, and hydrologists for forecast verification can be extended to prognostic forecasting, namely the concepts of skill, value, and reference predictions (Liang et al. 2011). Skill quantifies the performance improvement of the prognostic model over some reference model (perhaps scheduled maintenance), while value incorporates the cost savings of applying the prognostic method over the reference. One significant challenge in V\&V for NPP PHM systems is the lack of statistically sufficient data for testing the variety of algorithms applied across all SSCs. A rigorous V\&V methodology which utilizes available data and high-fidelity models specific to nuclear applications is needed with endorsement by the NRC.

\subsection{PHM Results Integration}

In addition to the research needed to fully develop and deploy accurate, reliable, robust PHM systems for NPPs, there is a need for research into how these results can be applied for practical improvements in plant safety, operations, and economics.

Traditional probabilistic risk assessment (PRA) is used to evaluate the risks associated with plant operations, such as taking a redundant system offline for maintenance. PRA can be used for riskinformed decision making, such as planning inservice testing, inservice inspection, and maintenance actions (Kafka 2002). There are two shortcomings in traditional PRA analysis that can be improved through the integration of PHM results. First, traditional PRA assumes that component failure rates are constant and do not depend on operating conditions, age, or unit-to-unit variation. However, a cursory review of the industry-reported data in the Reliability and Availability Data System (RADS) suggests that industry-wide values for the reliability and probability of failure for key plant components may not accurately reflect the reality at a specific plant (Rasmuson et al. 2005). Probability of failure distributions resulting from prognostic algorithms for both active and passive SSCs could be used to give a more 
accurate picture of overall risk for a specific plant. Second, PRA analysis currently does not incorporate most passive SSCs in risk analysis. As plants look at extended operating lives, these SSCs become more important to overall risk, especially when the relative difficulty and expense of replacing or repairing large passive structures is considered. Unfortunately, incorporating passive structures into PRA is not trivial. Early work in incorporating aging effects into PRA tended to focus on specific aging degradation mechanisms in only a few SSCs (Smith et al. 2001; Trifanov 2007). Initial research by Unwin et al. (2010; 2011b) looked at methods to intelligently incorporate the most risk-significant passive structures in PRA through expert analysis of features such as susceptibility (of a specific component to a specific degradation mechanism) and confidence (in the assessment of susceptibility). The modeling of aging passive component reliability was demonstrated for a dissimilar weld in a PWR primary coolant system (Unwin et al. 2011a). As monitoring and prognostic methods for passive components mature, accurate, component-specific reliability estimates can be used to better inform PRAs for passives.

In addition to incorporating PHM results into PRA for NPPs, RUL estimates can be used for O\&M planning and automated control. Integrating prognostics into control strategies and planning has been widely proposed (e.g., Gertsbakh 2000; Jardine and Tsang 2006; Liang et al. 2010b), but algorithms for effectively doing so have only recently been suggested (Liang et al. 2006; Tang et al. 2007; Liang et al. 2008; Edwards et al. 2010). In NPPs, safety is the primary goal in planning operations and maintenance. However, it is economically attractive to perform maintenance only at scheduled, convenient opportunities, such as planned refueling outages. The results of PHM systems could be incorporated into O\&M planning by determining if a faulted component can be operated under planned or reduced loads until the next convenient maintenance opportunity without affecting the safety margins of the plant. If the life of a faulted component would be extended, it would be beneficial to operate a plant at a reduced power output until the next planned outage rather than to shut down immediately for repairs to provide for full power capacity during operation. 


\subsection{Conclusions}

Recent years have seen a resurgence of nuclear power worldwide, with interest in maintaining and extending the safe, economic operation of the approximately 436 reactors currently in service (as of March 2012), 61 new reactors being constructed, and as many as 162 under consideration. The operating U.S. fleet includes 104 light water reactors. In addition, there are now (as of May 2012) four new AP-1000 nuclear plants under construction in the United States and two delayed plants being completed by the Tennessee Valley Authority. There is also interest in the United States in SMRs, most of which have longer operating periods between planned refueling and maintenance outages than currently operating LWRs. Renewed worldwide interest in nuclear power has been somewhat tempered by the March 2011 incident at Fukushima Dai-ichi in Japan. However, nuclear power is still considered a key element in meeting future worldwide sustainable energy, energy security, and emissions goals. Currently, three separate thrusts to safe and economic nuclear power development for energy security are being pursued in the United States: (i) longer term operation for the legacy fleet, considering operating lives of 60-80 years; (ii) near-term new nuclear plants with a 60-year design life; and (iii) small modular reactors, which are expected to employ light water reactor technology at least in the medium term. Within these activities, attention is turning to enhanced methods for plant component and structural health management.

Over the years, operational experience has shown that greater situational awareness of the state of safety-critical nuclear plant systems, structures, and components is necessary, particularly as they age over time due to exposure to harsh service conditions. While replacement of a subset of components is possible, it may be economically prohibitive to replace several of the larger components, such as the reactor pressure vessel or primary piping. Thus, detection, management, and mitigation of aging-related degradation in these critical components become important to maintain safety margins. In this context, the technical challenges related to detecting, characterizing, monitoring, and managing materials degradation need to be identified and addressed. The key technology developments necessary for detecting and managing degradation in reactor components are: (1) nondestructive measurement and analysis methods to detect degradation and anomalies, (2) algorithms to characterize and monitor the degradation state of the component, and (3) algorithms that use the degradation state information to determine remaining useful life (RUL) and probability of failure (POF) of the component. The POF information may then be used in a probabilistic risk assessment (PRA) model to assess the risk significance of the degradation and the corresponding reduced safety margin. Component health information may also be used to optimize operations and maintenance scheduling. Predictions of degradation growth rates can be coupled with prognostic algorithms and potential operation profiles to extend the life of degraded SSCs to the next convenient maintenance opportunity. Together, these technologies constitute prognostics and health management (PHM) systems. Ideally, degradation detection should occur early in the degradation development lifecycle to enable the application of appropriate mitigation or repair actions, thereby maintaining the necessary safety margins. Appropriate PHM systems therefore can potentially preclude serious consequences due to aging-related faults.

Prognostics and health management is a key need for improving the safety and economy of nuclear power generation moving forward with both LTO and new builds. The development of PHM systems to date has been largely driven by non-nuclear industries and applications, and adapting these developments to NPPs (either LWRs or advanced reactor designs) faces significant challenges. Several technical gaps exist, including in the area of sensors that can measure the features necessary to assess the health of SSCs; 
available data and first-principles models describing the complex degradation mechanisms in nuclear components; uncertainty quantification for sensor measurements, models, and RUL estimations; online assessment of prognostic model performance; and verification and validation of advanced models for PHM. Additionally, well-defined methods for incorporating the results of PHM algorithms into a more holistic view of plant operation, maintenance, and decision making are needed to provide a practical advantage for the use of PHM in terms of safety and economics. While some of these gaps are relevant to other areas and industries, the unique operational framework and licensing requirements of nuclear power pose additional challenges.

The domestic and international nuclear power industry poses a unique challenge for application of health management systems. NPPs worldwide are closely regulated by national and international licensing bodies, and application of PHM systems which may impact safety-critical systems will require a clear licensing basis outlining appropriate algorithms, architectures, and applications. In the United States, the licensing basis is developed by utilities; however, evaluation of such a licensing basis is the responsibility of the Nuclear Regulatory Commission (NRC). As PHM research and development progresses in the nuclear industry, it will be increasingly important to engage the NRC and other appropriate licensing and oversight bodies to enable deployment of these advanced methods in operating and future plants.

Clearly, PHM technologies can be beneficial to NPP operations, potentially providing improvements in safety, uptime, operations and maintenance optimization, and plant economics. These benefits are not unique to the operating fleet of LWRs, as the next generation of nuclear power reactors is expected to have similar needs in terms of managing and mitigating degradation; therefore, it is likely that any technology developed for detecting and characterizing degradation in aging LWRs will have applications beyond the current fleet. Additionally, some Gen III/III+, Gen IV, and SMR reactor designs will potentially have increased monitoring needs due to extended fuel cycles, reduced access to critical components, and remote siting with reduced maintenance staff. Lessons learned in operating and maintaining the existing fleet of NPPs indicates that new reactors will benefit from including advanced monitoring, fault detection, diagnostic, and prognostic infrastructure from initial design through end-oflife. It is likely that PHM systems in new reactor designs will need to be incorporated in the design phase instead of retrofitting health monitoring systems to existing plants, as this is more costly and likely more complicated. However, inclusion of PHM in new reactor designs will likely include additional challenges due to unique features of these advanced designs. While significant research has been performed in the areas of prognostics and health management, several gaps, both technological and regulatory, will need to be addressed before these methods are deployed in NPPs. In spite of these challenges, in both existing and future plants, the potential safety and economic incentives of applying PHM technologies are compelling. 


\subsection{References}

10 CFR 50.55a. 2007. "Codes and Standards." Code of Federal Regulations, U.S. Nuclear Regulatory Commission, Washington, D.C. Available at http://www.nrc.gov/reading-rm/doccollections/cfr/part050/part050-0055a.html.

10 CFR 50.65. 2011. "Requirements for Monitoring the Effectiveness of Maintenance at Nuclear Power Plants." Code of Federal Regulations, U.S. Nuclear Regulatory Commission, Washington, D.C. Available at http://www.nrc.gov/reading-rm/doc-collections/cfr/part050/part050-0065.html.

10 CFR 73.54. 2009. "Protection of Digital Computer and Communication Systems and Networks." Code of Federal Regulations, U.S. Nuclear Regulatory Commission, Washington, D.C. Available at http://www.nrc.gov/reading-rm/doc-collections/cfr/part073/part073-0054.html.

Abidi MA. 1992. Data Fusion in Robotics and Machine Intelligence. Academic Press.

Abram T and S Ion. 2008. "Generation-IV Nuclear Power: A Review of the State of the Science." Energy Policy 36(12):4323-4330.

Abu-Khader MM. 2009. "Recent Advances in Nuclear Power: A Review." Progress in Nuclear Energy 51:225-235.

Adams D, J White, M Rumsey and C Farrar. 2011. "Structural Health Monitoring of Wind Turbines: Method and Application to a HAWT." Wind Energy 14:603-623.

Ahmad R, S Banerjee and T Kundu. 2009. "Pipe Wall Damage Detection in Buried Pipes Using Guided Waves." Journal of Pressure Vessel Technology 131(1):011501-011510.

Ai Q, C-X Liu, X-R Chen, P He and Y Wang. 2010. "Acoustic Emission of Fatigue Crack in Pressure Pipe Under Cyclic Pressure." Nuclear Engineering and Design 240(10):3616-3620.

Anandakumaran K. 2007. "Aging and Condition Monitoring Studies of Composite Insulation Cables Used in Nuclear Power Plants." IEEE Transactions on Dielectrics and Electrical Insulation 14(1):227237.

Anderson MT, SL Crawford, SE Cumblidge, KM Denslow, AA Diaz and SR Doctor. 2007. Assessment of Crack Detection in Heavy-Walled Cast Stainless Steel Piping Welds Using Advanced Low-Frequency Ultrasonic Methods. NUREG/CR-6933, PNNL-16292, U.S. Nuclear Regulatory Commission, Washington, D.C.

Angeli C and A Chatzinikolaou. 2004. "On-Line Fault Detection Techniques for Technical Systems: A Survey." International Journal of Computer Science \& Applications I(1):12-30.

ANSI. Nuclear Energy Standards Coordination Collaborative. American National Standards Institute (ANSI). New York. Accessed March 13, 2012. Available at

http://www.ansi.org/standards_activities/standards_boards_panels/nescc/overview.aspx?menuid=3.

Arcella FG, FH Bednar, JJ Schreurs and JM Forker. 1994. "Online Valve Diagnostic Monitoring System." U.S. Patent 5,329,465. 
AREVA. 2007. OneproD MVX and MVP On-Line Systems. Product Literature, AREVA, Oilkiluoto 3, Finland.

AREVA. 2011. Press Release: EDF Chooses AREVA to Upgrade the Monitoring and Control Safety Systems for Its Twenty 1300MW Reactors. AREVA. Paris. Accessed March 13, 2012. Available at http://www.areva.com/EN/news-9125/edf-chooses-areva-to-upgrade-the-monitoring-and-control-safetysystems-for-its-twenty-1300mw-reactors.html.

AREVA. 2012. Operations: The EPR ${ }^{T M}$ Reactor Projects Worldwide. AREVA. Paris. Accessed March 13, 2012. Available at http://www.areva.com/EN/operations-2542/the-epr-reactors-under-constructionworldwide.html and links thereon.

Arunachalam K. 2006. Investigation of a Deformable Mirror Microwave Imaging and Therapy Technique for Breast Cancer. Ph.D. Thesis, Michigan State University, East Lansing, Michigan.

Arunachalam K, P Ramuhalli, L Udpa and SS Udpa. 2002. "Nonlinear Mixing Algorithms for Suppression of TSP Signals in Bobbin Coil Eddy Current Data." In Review of Progress in Quantitative Nondestructive Evaluation, Vol. 21, pp. 631-638. July 29-August 3, 2001, Brunswick, Maine. American Institute of Physics, Melville, New York.

Ayaz E. 2008. "Component-wide and Plant-Wide Monitoring by Neural Networks for Borssele Nuclear Power Plant." Energy Conversion and Management 49:3721-3728.

Ayaz E, S Seker, B Barutcu and E Turkcan. 2003. "Comparisons Between the Various Types of Neural Networks with the Data of Wide Range Operational Conditions of the Borssele NPP." Progress in Nuclear Energy 43(1-4):381-387.

Bailey C, C Yin, H Lu, M Musallam and CM Johnson. 2011. "Current Status of Prognostics Techniques and Application to Power Electronics." In 2010 6th International Conference on Integrated Power Electronics Systems (CIPS 2010). March 16-18, 2010, Nuremberg, Germany. IEEE Computer Society.

Balasubramanian S, B Shanker and L Udpa. 2001. "A Fast Integral Equation Based Scheme for Computing Magnetostatic Fields and Its Application to NDE Problems." In Review of Progress in Quantitative Nondestructive Evaluation Volume 21, pp. 331-337. July 29-August 3, 2001, Brunswick, Maine. American Institute of Physics, Melville, New York.

Banks J and J Merenich. 2007. "Cost Benefit Analysis for Asset Health Management Technology." In 2007 Annual Reliability and Maintainability Symposium (RAMS '07), pp. 95-100. January 22-25, 2007, Orlando, Florida. IEEE, Piscataway, New Jersey.

Barrett HH and KJ Myes. 2004. Foundations of Image Science. Wiley-Interscience.

Barron B. 2012. "Natural Gas vs. Nuclear; New Build vs Life Extension: A Discussion Around the NYISO Market." In Third International Conference on NPP Life Management (PLiM) for Long Term Operations (LTO). May 14-18, 2012, Salt Lake City, Utah. Keynote address.

Bartley WH. 2002. "Life Cycle Management of Utility Transformer Assets." Presented at Breakthrough Asset Management for the Restructured Power Industry, October 10-11, 2002, Salt Lake City, Utah. Available at http://www.bplglobal.net/eng/knowledge-center/download.aspx?id=196. 
Bartley WH. 2003. "Analysis of Transformer Failures." Presented at International Association of Engineering Insurers, 36th Annual Conference - Stockholm, 2003. Paper IMIA WGP33. Available at http://www.bplglobal.net/eng/knowledge-center/download.aspx?id=191.

Barutcu B, S Seker, E Ayaz and E Turkcan. 2003a. "Real Time Reactor Noise Diagnostics for the Borssele (PWR) Nuclear Power Plant." Progress in Nuclear Energy 43(1-4):137-143.

Barutcu B, E Turkcan, S Seker and E Ayaz. 2003b. "On-Line Identification of the Nuclear Power Plant Physical Parameters at Power Operation." In Third International Conference on Electrical and Electronics Engineering (ELECO’2003), pp. 158-162. December 3-7, 2003, Bursa, Turkey.

Baskaran G, K Balasubramaniam, CV Krishnamurthy and CL Rao. 2004. "TOFD IMAGING: Ultrasonic TOFD Flaw Sizing and Imaging in Thin Plates Using Embedded Signal Identification Technique (ESIT)." Insight - Non-Destructive Testing and Condition Monitoring 49(6):537-542.

Basseville M, A Benveniste, M Goursat and L Mevel. 2007. "Subspace-Based Algorithms for Structural Identification, Damage Detection and Sensor Data Fusion." EURASIP Journal on Advances in Signal Processing 2007(1):069136.

Bates DJ, SR Doctor, PG Heasler and E Burck. 1987. Stainless Steel Round Robin Test: Centrifugally Cast Stainless Steel Screening Phase. NUREG/CR-4970, PNL-6266, PISC III Report No. 3, U.S. Nuclear Regulatory Commission, Washington, D.C.

Bencharit U, JL Kaufman, NM Bilgutay and J Saniie. 1986. "Frequency and Spatial Compounding Techniques for Improved Ultrasonic Imaging." In IEEE 1986 Ultrasonics Symposium, pp. 1021-1026. November 17-19, 1986, Williamsburg, Virginia. IEEE, New York.

Benson J, S Ramakrishnan, S Majumdar, P Ramuhalli, L Udpa and S Udpa. 2007. "Automated Analysis Systems for Characterizing Eddy Current SG Inspection Data." In The13th International Symposium on Applied Electromagnetics and Mechanics (ISEM 2007), pp. 157-158. September 9-12, 2007, East Lansing, Michigan.

Berens AP and PW Hovey. 1981. Evaluation of NDE Reliability Characterization. AFWAL-TR-814160, Vol. I, Materials Laboratory, Air Force Wright Aeronautical Laboratories, Wright-Patterson Air Force Base, Ohio.

Berens AP and PW Hovey. 1983. "Statistical Methods for Estimating Crack Detection Probabilities." In Probabilistic Fracture Mechanics and Fatigue Methods: Applications for Structural Design and Maintenance, pp. 79-94 ed: E Bloom. ASTM International, West Conshohocken, Pennsylvania. ASTM Special Technical Publication No. 798.

Bickford R, E Davis, R Rusaw and R Shankar. 2002. "Development of an Online Predictive Monitoring System for Power Generating Plants." In 45th ISA POWID. June 3-5, 2002, San Diego, California. Paper No. 805.

Bilgutay NM, U Bencharit and J Saniie. 1989. "Enhanced Ultrasonic Imaging with Split Spectrum Processing and Polarity Thresholding." IEEE Transactions on Acoustics, Speech and Signal Processing 37(10):1590-1592. 
Bisconti AS. 2007. "Public Supports Climate Change Action, But Is Unclear on Nuclear Energy's Role in Preventing Greenhouse Gases." Prepared for the Nuclear Energy Institute.

http://www.nei.org/filefolder/popo-may.pdf.

Bond LJ. 1999. "Predictive Engineering for Aging Infrastructure." In Nondestructive Evaluation of Utilities and Pipelines III, Proceedings of SPIE, pp. 2-13. March 4, 1999, Newport Beach, California.

Bond LJ. 2010. "Moving Beyond NDE to Proactive Management of Materials Degradation." In Proceedings of the ASME 2010 Pressure Vessels \& Piping Division / K-PVP Conference (PVP2010). July 18-22, 2010, Bellevue, Washington. American Society of Mechanical Engineers, New York.

Bond LJ, J Coble and JWD Bond. 2012 (draft). Economics of On-line Monitoring and Nuclear Power Plant Life Extension. Pacific Northwest National Laboratory, Richland, Washington. In draft.

Bond LJ, SR Doctor, SM Bruemmer, SE Cumblidge, AB Hull and SN Malik. 2009a. "Failure Prevention for Nuclear Power Plants through Proactive Management of Materials Degradation (PMMD)." In Proceedings, MFPT 2009, Failure Prevention: Implementation, Success Stories and Lessons Learned, pp. 389-408. April 28-30, 2009, Dayton, Ohio. Society of Machinery Failure and Prevention Technology (MFPT), The Vibration Institute, Dayton, Ohio.

Bond LJ, SR Doctor, SE Cumblidge, SM Bruemmer, WB Taylor, AB Hull and SN Malik. 2009b. "LWR Aging Management Using a Proactive Approach to Control Materials Degradation." In Proceedings, International Congress on Advances in Nuclear Power Plants (ICAPP 2009). May 10-14, 2009, Tokyo.

Bond LJ, SR Doctor, JW Griffin, AB Hull and SN Malik. 2009c. "Damage Assessment Technologies for Prognostics and Proactive Management of Materials Degradation (PMMD)." In 6th International Topical Meeting on Nuclear Plant Instrumentation, Control, and Human-Machine Interface Technologies. April 5-9, 2009, Knoxville, Tennessee. American Nuclear Society, La Grange Park, Illinois.

Bond LJ, SR Doctor, JW Griffin, AB Hull and SN Malik. 2011a. "Damage Assessment Technologies for Prognostics and Proactive Management of Materials Degradation." Nuclear Technology 173(1):46-55.

Bond LJ, SR Doctor, DB Jarrell and JWD Bond. 2008a. "Improved Economics of Nuclear Plant Life Management." In Second International Symposium on Nuclear Power Plant Life Management. October 15-18, 2007, Shanghai, China. International Atomic Energy Agency, Vienna, Austria. IAEA Paper IAEA-CN-155-008KS (in press).

Bond LJ, SR Doctor, DB Jarrell and JWD Bond. 2008b. "Improved Economics of Nuclear Plant Life Management." In Second International Symposium on Nuclear Power Plant Life Management. October 15-18, 2007, Shanghai, China. International Atomic Energy Agency. IAEA Paper IAEA-CN-155$008 \mathrm{KS}, 22 \mathrm{pp}$.

Bond LJ, SR Doctor and TT Taylor. 2008c. Proactive Management of Materials Degradation - A Review of Principles and Programs. PNNL-17779, Pacific Northwest National Laboratory, Richland, Washington.

Bond LJ and RM Meyer. 2011. "Online Monitoring to Enable Improved Diagnostics, Prognostics and Maintenance." In International Symposium on Future I\&C for Nuclear Power Plants, Cognitive Systems Engineering in Process Control, International Symposium on Symbiotic Nuclear Power Systems (ICI 2011). August 21-25, 2011, Daejeon, Korea. 
Bond LJ, P Ramuhalli, MS Tawfik and NJ Lybeck. 2011b. "Prognostics and Life Beyond 60 Years for Nuclear Power Plants." In Proceedings of the 2011 IEEE International Conference on Prognostics and Health Management, pp. 1-7. June 20-23, 2011, Denver, Colorado.

Bond LJ, TT Taylor, SR Doctor, AB Hull and SN Malik. 2008d. "Proactive Management of Materials Degradation (PHM 2008)." In International Conference on Prognostics and Health Management 2008. October 6-9, 2008, Denver, Colorado. IEEE, Piscataway, New Jersey.

Braverman JI, G DeGrassi, G Martinez-Guridi, RJ Morante and CH Hofmayer. 2005. Risk-Informed Assessment of Degraded Buried Piping Systems in Nuclear Power Plants. NUREG/CR-6876, BNLNUREG-74000-2005), U.S. Nuclear Regulatory Commission, Washington, DC.

Brown DW, PW Kalgren, CS Byington and MJ Roemer. 2007. "Electronic Prognostics - A Case Study Using Global Positioning System (GPS)." Microelectronic Reliability 47:1874-1881.

Byington CS, M Watson, D Edwards and P Stoelting. 2004. "A Model-Based Approach to Prognostics and Health Management for Flight Control Actuators." In 2004 IEEE Aerospace Conference, pp. 35513562. March 6-13, 2004, Big Sky, Montana. IEEE, Piscataway, New Jersey.

Callan R, B Larder and J Sandiford. 2006. "An Integrated Approach to the Development of an Intelligent Prognostic Health Management System." In 2006 IEEE Aerospace Conference. March 4-11, 2006, Big Sky, Montana. IEEE, Piscataway, New Jersey.

Caorsi S, GL Gragnani, S Medicina, M Pastorino and G Zunino. 1994. "Microwave Imaging Based on a Markov Random Field Model." IEEE Transactions on Antennas and Propagation 42(3):293-303.

Carnahan D, D Chung, E DelaHostria and C Hoover. 2005. "Integration of Production, Diagnostics, Capability Assessment, and Maintenance Information Using ISO 18435." In ISA EXPO 2005 Technical Conference, pp. 718-729. October 25-27, 2005, Chicago, Illinois. Instrumentation, Systems, and Automation Society (ISA).

Carter JP. 2006. "The Transformation of the Nuclear Power Industry - From Boom, To Bust, To Quiet Optimism." IEEE Power and Energy Magazine 4(6):25-33.

Catterson VM, EM Davidson and SDJ McArthur. 2005. "Issues in Integrating Existing Multi-Agent Systems for Power Engineering Applications." In 13th International Conference on Intelligent Systems Application to Power Systems (ISAP'05), pp. 396-401. November 6-10, 2005, Arlington, Virginia. IEEE, Piscataway, New Jersey.

Catterson VM, SDJ McArthur, MD Judd and AS Zaher. 2008. "Managing Remote Online Partial Discharge Data." IEEE Transactions on Power Delivery 23(4):1754-1762.

Catterson VM, SE Rudd, SDJ McArthur and G Moss. 2009. "On-line Transformer Condition Monitoring Through Diagnostics and Anomaly Detection." In 2009 15th International Conference on Intelligent System Applications to Power Systems (ISAP '09). November 8-12, 2009, Curitiba, Brazil. IEEE Computer Society, Piscataway, New Jersey.

Cerafici TJ. 2009. "Is New Always Better? The Case for License Renewal in the Next Generation." Pace Environmental Law Review 26(2):Article 4. 
Chai J, RH Lyon and JH Lang. 1994. "Non-invasive Diagnostics of Motor-operated Valves." In Proceedings of 1994 American Control Conference (ACC '94), pp. 2006-2012. June 29-July 1, 1994, Baltimore, Maryland. IEEE, Piscataway, New Jersey.

Chen J, Y Shi and S Shi. 1999. "Noise Analysis of Digital Ultrasonic Nondestructive Evaluation System." International Journal of Pressure Vessels and Piping 76(9):619-630.

Chen Z and S Zheng. 2005. "Lifetime Distribution Based Degradation Analysis." IEEE Transactions on Reliability 54(1):3-10.

Cheng S and M Pecht. 2009. "A Fusion Prognostics Method for Remaining Useful Life Prediction of Electronic Products." In 2009 IEEE International Conference on Automation Science and Engineering (CASE 2009), pp. 102-107. August 22-25, 2009, Bangalore, India. IEEE Computer Society.

Chinnam RB. 1999. "On-line Reliability Estimation of Individual Components, Using Degradation Signals." IEEE Transactions on Reliability 48(4):403-412.

Chockie AD, KA Bjorkelo, TE Fleming, WB Scott and WI Enderlin. 1991. Maintenance Practices to Manage Aging: A Review of Several Technologies. PNL-7823, Pacific Northwest Laboratory, Richland, Washington.

Chockie LJ. 1981. "PVRC Round Robin Ultrasonic Program, Results and Assessment of Reliability." In Nondestructive Evaluation in the Nuclear Industry -- 1980, pp. 361-379. February 11-13, 1980, Salt Lake City, Utah. American Society for Metals, Metals Park, Ohio.

Chockie LJ. 1985. "Section XI of the ASME Code A New Approach to Qualifying Procedures and Personnel." In 7th International Conference on NDE in the Nuclear Industry, pp. 83-86 Grenoble, France.

Choi MH. 2007. "Spatial Compounding from Ultrasonic RF Data for Speckle Reduction in Ultrasonic Diagnostic Imaging System." In International Conference on Control, Automation and Systems, ICCAS 2007, pp. 1577-1580. October 17-20, 2007, Seoul, Korea. IEEE Computer Society, Piscataway, New Jersey.

Clayton D and R Wood. 2011. "The Role of I\&C Technology in Enabling the Deployment of Small Modular Reactors." Nuclear News 54(13):42-47.

Coble JB. 2010. Merging Data Sources to Predict Remaining Useful Life - An Automated Method to Identify Prognostic Parameters. Ph.D. Thesis, University of Tennessee, Knoxville, Tennessee. Available at http://trace.tennessee.edu/utk_graddiss/683.

Coble JB and JW Hines. 2008. "Prognostic Algorithm Categorization with PHM Challenge Application." In 1st International Conference on Prognostics and Health Management (PHM 2008), pp. 1-11. October 6-9, 2008, Denver, Colorado. IEEE, Piscataway, New Jersey.

Coble JB and JW Hines. 2011. "Applying the General Path Model to Estimation of Remaining Useful Life." International Journal of Prognostics and Health Management 2(1). http://www.phmsociety.org/node/154. 
Coble JB, E Strong, S O'Reilly and JW Hines. 2011. "Prognosis of Remaining Useful Life for Large, Oil-Filled Transformers." In 2011 ANS Winter Meeting and Nuclear Technology Expo - The Status of Global Nuclear Deployment. October 30-November 3, 2011, Washington, D.C.

Colton D and A Kirsch. 1996. "A Simple Method for Solving Inverse Scattering Problems in the Resonance Region." Inverse Problems 12:383-393.

Colton D and R Kress. 1998. Inverse Acoustic and Electromagnetic Scattering Theory. Springer-Verlag.

Connolly GD, MJS Lowe, SI Rokhlin and JAG Temple. 2009. "Imaging of Simple Defects in Austenitic Steel Welds Using a Simulated Ultrasonic Array." In Proceedings of the 35th Annual Review of Progress in Quantitative Nondestructive Evaluation, pp. 880-887. July 20-25, 2008, Chicago, Illinois. American Institute of Physics, Melville, New York.

Cox DR and D Oakes. 1984. Analysis of Survival Data, Monographs on Statistics and Applied Probability 21. Chapman \& Hall/CRC, Boca Raton, Florida.

Cumblidge SE, MT Anderson and SR Doctor. 2004. An Assessment of Visual Testing. NUREG/CR6860, U.S. Nuclear Regulatory Commission, Washington, D.C.

Cumblidge SE, MT Anderson, SR Doctor, FA Simonen and AJ Elliot. 2007. A Study of Remote Visual Methods to Detect Cracking in Reactor Components. NUREG/CR-6943, PNNL-16472, U.S. Nuclear Regulatory Commission, Washington, D.C.

Cumblidge SE, SR Doctor, LJ Bond, TT Taylor, TR Lupold, AB Hull and SN Malik. 2009. "Analysis of Emerging NDE Techniques - Methods for Evaluating and Implementing Continuous Online Monitoring." In Proceedings of the 17th International Conference on Nuclear Engineering 2009, ICONE17. July 1216, 2009, Brussels, Belgium. American Society of Mechanical Engineers, New York.

Daigle M, I Roychoudhury, S Narasimhan, S Saha, B Saha and K Goebel. 2011. "Investigating the Effect of Damage Progression Model Choice on Prognostics Performance." In Annual Conference of the Prognostics and Health Management Society 2011. September 25-29, 2011, Montreal, Quebec.

Dale CJ. 1985. "Application of the Proportional Hazards Model in the Reliability Field." Reliability Engineering 10(1):1-14.

Darty M, S Li Pi and C Bosco. 1994. "Built-in Diagnostics for Advanced Power Management." In AUTOTESTCON '94 - IEEE Systems Readiness Technology Conference, pp. 399-407. September 20-22, 1994, Anaheim, California. IEEE, Piscataway, New Jersey.

Dasarathy BV. 1997. "Sensor Fusion Potential Exploitation - Innovative Architectures and Illustrative Applications." Proceedings of the IEEE 85(1):24-38.

Davis E, R Bickford, P Colgan, K Nesmith, R Rusaw and R Shankar. 2002. "On-Line Monitoring at Nuclear Power Plants - Results from the EPRI On-Line Monitoring Implementation Project." In 45th ISA POWID. June 3-5, 2002, San Diego, California.

Demo J, C Andrews, F Friedersdorf and M Putic. 2011. "Diagnostics and Prognostics for Aircraft Structures Using a Wireless Corrosion Monitoring Network." In 2011 IEEE Aerospace Conference. March 5-12, 2011, Big Sky, Montana. IEEE, Piscataway, New Jersey. 
Diaz NJ. 2004. The 3rd Annual Homeland Security Summit; Session on, "The Best-Laid Plans: A Case Study in Preparedness Planning": The Very Best-Laid Plans (the NRC's Defense-in Depth Philosophy). U.S. Nuclear Regulatory Commission. Washington, D.C. Available at http://www.nrc.gov/readingrm/doc-collections/commission/speeches/2004/s-04-009.html.

Dion J, MK Howlader and PD Ewing. 2010. "Wireless Network Security in Nuclear Facilities." In 7th International Topical Meeting on Nuclear Plant Instrumentation, Control, and Human-Machine Interface Technologies (NPIC\&HMIT 2010), pp. 567-578, Vol. 1. November 7-11, 2010, Las Vegas, Nevada. American Nuclear Society, LaGrange Park, Illinois.

Dion J, M Kumar and P Ramuhalli. 2007. "Multisensor Data Fusion for High-Resolution Material Characterization." In Review of Progress in Quantitative Nondestructive Evaluation, pp. 1189-1196. July 30-August 4, 2006, Portland, Oregon. American Institute of Physics, Melville, New York.

Dobmann G. 2006. "NDE for Material Characterization of Aging Due to Thermal Embrittlement, Fatigue and Neutron Degradation." International Journal of Materials and Product Technology 26:122139.

Doctor SR. 1984. "NDE Reliability Assessment." In Non-Destructive Examination for Pressurised Components, pp. 323-335. August 30-31, 1983, Monterey, California. Elsevier Applied Science Publishers, London.

Doctor SR. 1988. "Measurement Challenges Associated with Irradiated Reactor Components." In Nondestructive Characterization of Materials in Aging Systems, Materials Research Society Symposium Proceedings, pp. 163-168 Boston, Massachusetts. Materials Research Society.

Doctor SR. 2007. "Nuclear Power Plant NDE Challenges - Past, Present, and Future." In Review of Progress in Quantitative Nondestructive Evaluation, pp. 17-31. July 30-August 4, 2006, Portland, Oregon. DOI 10.1063/1.2717950. American Institute of Physics, Melville, New York.

Doctor SR, LJ Bond, SE Cumblidge, SM Bruemmer, WB Taylor, CE Carpenter, AB Hull and SN Malik. 2010. "Aging Management Using Proactive Management of Materials Degradation." In Proceedings of Review of Progress in Quantitative Nondestructive Evaluation, pp. 1876-1883. July 26-31, 2009, Kingston, Rhode Island. American Institute of Physics, Melville, New York.

Doctor SR, P Lemaitre and S Crutzen. 1995. "Austenitic Steel Piping Testing Exercises in PISC." Nuclear Engineering and Design 157(1-2):231-44.

Droste D and K Finklea. 2006. "Prognostics for Electronic Systems." In AUTOTESTCON 2006 - IEEE Systems Readiness Technology Conference, pp. 181-186. September 18-21, 2006, Anaheim, California. IEEE, Piscataway, New Jersey.

Drummond C and C Yang. 2008. "Reverse-Engineering Costs: How Much will a Prognostic Algorithm Save?" In 1st International Conference on Prognostics and Health Management. October 6-9, 2008, Denver, Colorado.

Duc LM and TC Ming. 2011. "Cost Comparison of Maintenance Policies." In Annual Conference of the Prognostics and Health Management Society. September 25-29, 2011, Montreal, Quebec, Canada. PHM Society. 
Edwards D, ME Orchard, L Tang, K Goebel and G Vachtsevanos. 2010. "Impact of Input Uncertainty on Failure Prognostic Algorithms: Extending the Remaining Useful Life of Nonlinear Systems." In Annual Conference of the Prognostics and Health Management Society, 2010. October 13-16, 2010, Portland, Oregon.

Engel SJ, BJ Gilmartin, K Bongort and A Hess. 2000. "Prognostics, the Real Issues Involved with Predicting Life Remaining." In 2000 IEEE Aerospace Conference, pp. 457-469. March 18-25, 2000, Big Sky, Montana. IEEE, Piscataway, New Jersey.

EPRI. 1998. On-Line Monitoring of Instrument Channel Performance. Report No. 104965, Electric Power Research Institute (EPRI), Palo Alto, California.

EPRI. 2007. Guidelines for Inspectability for New Plant Components. Product ID Number 1015139, Electric Power Research Institute, Palo Alto, California.

EPRI. 2008a. Automated Analysis of Array Probe Eddy Current Data. Report 1015125, Electric Power Research Institute, Inc. (EPRI), Palo Alto, California.

EPRI. 2008b. Recommendations for an Effective Program to Control the Degradation of Buried Pipe. Report No. 1016456, Electric Power Research Institute (EPRI), Palo Alto, California.

EPRI. 2008c. TR-105696-R11 (BWRVIP-03) Revision 11: BWR Vessel and Internals Project, Reactor Vessel and Internals Examination Guidelines TR-1016584, Electric Power Research Institute, Palo Alto, California.

EPRI. 2009a. Program on Technology Innovation: Prognostic Application for the Power Industry. Report 1018245, Electric Power Research Institute, Inc. (EPRI), Palo Alto, California.

EPRI. 2009b. Steam Generator Management Program: Automated Analysis Performance Demonstration Database. Report 1019293, Electric Power Research Institute, Inc. (EPRI), Palo Alto, California.

EPRI. 2010. Long-Term Nuclear Operations Executive Summary. Product ID 1021530, Electric Power Research Institute, Inc. (EPRI), Palo Alto, California. Available at http://mydocs.epri.com/docs/SummerSeminar10/brochures/Issue_Nuclear-20100726A_small.pdf.

EPRI. 2011a. "EPRI On-line Monitoring and APR Technology Implementation Workshop for Nuclear Generating Companies." Electric Power Research Institute, Palo Alto, California. Materials from EPRI's January 2011 workshop held in Atlanta, Georgia.

EPRI. 2011b. Ginna NGS Containment Monitoring Feasibility Study. Electric Power Research Institute, Palo Alto, California.

EPRI. 2011c. Materials Reliability Program: Pressurized Water Reactor Internals Inspection and Evaluation Guidelines (MRP-227-A) TR-1022863, Electric Power Research Institute, Palo Alto, California.

EPRI. 2011d. Program on Technology Innovation: Equipment Monitoring via Transient Methods - A Polaris Initiative Project. TR 1022582, Electric Power Research Institute, Palo Alto, California. 
Ericsson L. 1994. Reduction of Material Noise in Ultrasonic Nondestructive Evaluation Using Synthetic Frequency Diversity Algorythms. Thesis, Teknikum Institute of Technology, Uppsala University, Uppsala, Sweden.

Eryurek E and E Turkcan. 1991. Neural Networks for Sensor Validation and Plant-Wide Monitoring. ECN-RX-91-089, ECN, Petten, Switzerland.

Fang W and D Guanzhong. 2010. "Research and Application of Multi-agent Model for Aircraft PHM." In 2010 2nd IEEE International Conference on Information Management and Engineering (ICIME 2010). April 16-18, 2010, Chengdu, China. IEEE, Piscataway, New Jersey.

Fantoni P and GJ Toman. 2006. "Wire System Aging Assessment and Condition Monitoring Using Line Resonance Analysis (LIRA)." In Offshore Wind and Other Marine Renewable Energies in Mediterranean and European Seas (OWEMES). April 20-22, 2006, Citavecchia (Rome), Italy.

Fantoni PF. 2005. "Experiences and Applications of PEANO for Online Monitoring in Power Plants." Progress in Nuclear Energy 46(2-3):206-225.

Fantoni PF. 2010. "Cable Aging Assessment." In Report from the Light Water Reactor Sustainability Workshop on On-Line Monitoring Technologies, pp. 47-53. June 10-12, 2010, Seattle, Washington. Idaho National Laboratory, Idaho Falls, Idaho. Report No. INL/EXT-10-19500.

Fantoni PF, GJ Toman and JC Cano. 2009. "Condition Monitoring of Electrical Cables using Line Resonance Analysis (LIRA)." In ANS NPIC\&HMIT 2009 Topical Meeting - Nuclear Plant Instrumentation, Controls, and Human Machine Interface Technology. April 5-9, 2009, Knoxville, Tennessee. American Nuclear Society, LaGrange Park, Illinois.

Ferrell BL. 1999. "JSF Prognostics and Health Management." In 1999 IEEE Aerospace Conference, p. 471 Vol. 2. March 6-13, 1999, Aspen, Colorado. IEEE, Piscataway, New Jersey.

Ferrell BL. 2000. "Air Vehicle Prognostics and Health Management." In 2000 IEEE Aerospace Conference, pp. 145-146. March 18-25, 2000, Big Sky, Montana. IEEE, Piscataway, New Jersey.

Fong JT. 1986. NDE Reliability through Round Robin Testing: Presented at the 4th National Congress on Pressure Vessels and Piping Technology, Portland, Oregon, June 19-24, 1983 and the 1986 Pressure Vessels and Piping Conference and Exhibition, Chicago, Illinois, July 20-24, 1986. American Society of Mechanical Engineers, New York.

Forsyth D and J Komorowski. 2000. "Role of Data Fusion in NDE for Aging Aircraft." In Proceedings of SPIE - Nondestructive Evaluation of Aging Aircraft, Airports, and Aerospace Hardware IV, pp. 47-58. March 7-8, 2000, Newport Beach, California. Society of Photo-Optical Instrumentation Engineers, Bellingham, Washington.

GAO. 2011. NUCLEAR REGULATORY COMMISSION: Oversight of Underground Piping Systems Commensurate with Risk, but Proactive Measures Could Help Address Future Leaks. GAO-11-563, U.S. Government Accountability Office (GAO), Washington, D.C.

Garvey D, J Garvey, R Seibert, JW Hines and SA Arndt. 2006. "Application of On-Line Monitoring Techniques to Nuclear Plant Data." In 5th International Topical Meeting on Nuclear Plant Instrumentation Controls and Human Machine Interface Technology (NPIC\&HMIT 2006). November 12-16, 2006, Albuquerque, New Mexico. American Nuclear Society, LaGrange Park, Illinois. 
Garvey DR and JW Hines. 2006. "The Development of a Process and Equipment Monitoring (PEM) Toolbox and its Application to Sensor Calibration Monitoring." Quality and Reliability Engineering International 22:1-13.

Garvey J, D Garvey, R Seibert and JW Hines. 2007a. "Nuclear Power Plant On-Line Sensor Calibration Monitoring Implementation Issues." International Journal of Nuclear Knowledge Management 2(3):204223.

Garvey J, D Garvey, R Seibert and JW Hines. 2007b. "Validation of On-Line Monitoring Techniques to Nuclear Plant Data." Nuclear Engineering and Technology 39(2):149-158.

Gertler JJ. 1988. "Survey of Model-based Failure Detection and Isolation in Complex Plants." IEEE Control Systems 8(6):3-11.

Gertsbakh I. 2000. Reliability Theory: With Applications to Preventive Maintenance. Springer, Berlin.

Ghoshal G and JA Turner. 2009. "Diffuse Ultrasonic Backscatter in a Two-Dimensional Domain." Acta Mechanica 205:35-49.

Ghouti L. 1997. "High-Order Spectra-Based Deconvolution of Ultrasonic NDT Signals for Defect Identification." Ultrasonics 35(17):525-531.

Giordano PJ and D Carey. 1995. "A New Breed of Smart Depot Testers Using COTS Technology." In AUTOTESTCON'95 - Systems Readiness: Test Technology for the 21st Century Conference, pp. 300307. August 8-10, 1995, Atlanta, Georgia. IEEE, Piscataway, New Jersey.

Giordano PJ and F Levy. 1994. "Re-engineeering the Test Development Process." In AUTOTESTCON '94 - IEEE Systems Readiness Technology Conference, pp. 527-535. September 20-22, 1994, Anaheim, California. IEEE, Piscataway, New Jersey.

Girish T, SW Lam and SR Jayaram. 2003. "Reliability Prediction Using Degradation Data - A Preliminary Study Using Neural Network-Based Approach." In European Safety \& Reliability International Conference (ESREL 2003), pp. 681-688. June 15-18, 2003, Maastricht, Netherlands.

Goodman DL. 2000. Prognostic Techniques for Semiconductor Failure Modes. Ridgetop Group, Inc., Tucson, Arizona. Available at http://www.ridgetopgroup.com/doc/WP-

electronic_prognostic techniques.pdf.

Goodman DL, B Vermeire, J Good-Ralston and R Graves. 2006. "A Board-Level Prognostic Monitor for MOSFET TDDB." In 2006 IEEE Aerospace Conference. March 4-11, 2006, Big Sky, Montana. IEEE, Piscataway, New Jersey.

Granjon P. 2011. "Condition Monitoring of Motor-Operated Valves in Nuclear Power Plants." In 8th International Conference on Condition Monitoring and Machinery Failure Prevention Technologies (CM/MFPT 2011), pp. 109-119. June 20-22, 2011, Cardiff, Wales. Curran Assoc., Red Hook, New York.

Gregor F and A Chockie. 2006. Performance Monitoring of Systems and Active Components. CGI Report 06:21, Chockie Group International, Inc., Seattle, Washington. 
Greitzer FL. 2000. "Life Extension Analysis and Prognostics Architectures." In Laboratory Directed Research and Development Annual Report, Fiscal Year 1999 (PNNL-13203), pp. 85-88. Pacific Northwest National Laboratory, Richland, Washington.

Greitzer FL. 2001a. "Life Extension Analysis and Prognostics (LEAP) Architectures." In Laboratory Directed Research and Development Annual Report, Fiscal Year 2000 (PNNL-13501), pp. 447-450. Pacific Northwest National Laboratory, Richland, Washington.

Greitzer FL. 2001b. "Life Extension Analysis and Prognostics (LEAP) Architectures." In Laboratory Directed Research and Development Annual Report, Fiscal Year 2001 (PNNL-13855), pp. 312-316. Pacific Northwest National Laboratory, Richland, Washington.

Greitzer FL and T Ferryman. 2001. "Predicting Remaining Life of Mechanical Systems." In ASNE Intelligent Ship Symposium IV. April 2-3, 2001, Philadelphia, Pennsylvania. American Society of Naval Engineers, Alexandria, Virginia.

Greitzer FL, LJ Kangas and M Melius. 1997. "An Automated Health Monitoring System for the Army M1A1/A2 Main Battle Tank AGT-1500 Gas Turbine Engine." In USAF Inaugural Engine Condition Monitoring (ECM) Workshop. October 21-23, 1997, San Diego, California.

Greitzer FL, LJ Kangas, KM Terrones, MA Maynard, BW Wilson, RA Pawlowski, DR Sisk and NB Brown. 1999a. "Gas Turbine Engine Health Monitoring and Prognostics." In International Society of Logistics (SOLE) 1999 Symposium. August 30-September 2, 1999, Las Vegas, Nevada.

Greitzer FL and RA Pawlowski. 2002. "Embedded Prognostics Health Monitoring." In Proceedings of the 48th International Instrumentation Symposium, pp. 301-310. May 5-9, 2002, San Diego, California. Instrumentation, Systems, and Automation Society (ISA). PNNL-SA-35920.

Greitzer FL, EJ Stahlman, TA Ferryman, BW Wilson, LJ Kangas and DR Sisk. 1999b. "Development of a Framework for Predicting Life of Mechanical Systems: Life Extension Analysis and Prognostics (LEAP) " In International Society of Logistics (SOLE) 1999 Symposium, pp. 1-7. August 30-September 2, 1999, Las Vegas, Nevada. SOLE, Hyattsville, Maryland.

Griffith G, R Youngblood, J Busby, B Hallbert, C Barnard and K McCarthy. 2012. Light Water Reactor Sustainability Program Integrated Program Plan. INL/EXT-11-23452, Idaho National Laboratory, Idaho Falls, Idaho.

Gros XE. 1997. NDT Data Fusion. Edward Arnold, London.

Gross KC, V Bhardwaj and R Bickford. 2003. "Proactive Detection of Software Aging Mechanisms in Performance Critical Computers." In 27th Annual NASA Goddard/IEEE Software Engineering Workshop, pp. 17-23. December 5-6, 2002, Greenbelt, Maryland. IEEE Computer Society.

Gross KC, RM Singer and KE Humenik. 1993. "Expert System for Online Surveillance of Nuclear Reactor Coolant Pumps." U.S. Patent 5,223,207.

Gross KC, RM Singer, SW Wegerich, JP Herzog, R VanAlstine and F Bockhorst. 1997. "Application of a Model-Based Fault Detection System to Nuclear Power Signals." In Proceedings of 1997 Conference on Intelligent System Applications to Power Systems, pp. 66-70. July 6-10, 1997, Seoul, South Korea. Korean Institute of Electrical Engineering. 
Gross KC, K Whisnant and A Urmanov. 2006. "Electronic Prognostics Through Continuous System Telemetry." In Proceedings of the 60th Machinery Failure Prevention Technology (MFPT) Conference, pp. 53-62. April 3-6, 2006, Virginia Beach, Virginia. Society Machinery Failure Prevention Technology.

Gu J, N Vichare, T Tracy and M Pecht. 2007. "Prognostics Implementation Methods for Electronics." In 2007 Annual Reliability and Maintainability Symposium (RAMS '07), pp. 101-106. January 22-25, 2007, Orlando, Florida. IEEE, Piscataway, New Jersey.

Hall DL and J Llinas. 1997. "An Introduction to Multisensor Data Fusion." Proceedings of the IEEE 85(1):6-23.

Hameed Z, SH Ahn and YM Cho. 2010. "Practical Aspects of a Condition Monitoring System for a Wind Turbine with Emphasis on Its Design, System Architecture, Testing and Installation." Renewable Energy 35:879-894.

Hameed Z, YS Hong, YM Cho, SH Ahn and CK Song. 2009. "Condition Monitoring and Fault Detection of Wind Turbines and Related Algorithms: A Review." Renewable and Sustainable Energy Reviews 13:1-39.

Hargreaves ML. 1988. Digital Processing of Ultrasound Signals Back-scattered from Coarse Grained Austenitic Stainless Steel. Ph.D. Thesis, University of Keele, Keele, Staffordshire, England.

Harris DO and HL Dunegan. 1974. "Continuous Monitoring of Fatigue-Crack Growth by AcousticEmission Techniques." Experimental Mechanics 14(2):71-81.

Hashemian H. 1995. On-line Testing of Calibration of Process Instrumentation Channels in Nuclear Power Plants. NUREG/CR-6343, U.S. Nuclear Regulatory Commission, Washington, D.C.

Hashemian H. 2011a. "Wireless Sensors for Predictive Maintenance of Rotating Equipment in Research Reactors." Annals of Nuclear Energy 38:665-680.

Hashemian H and MA Feltus. 2006. "On-Line Condition Monitoring Applications in Nuclear Power Plants." In 5th International Topical Meeting on Nuclear Plant Instrumentation Controls and Human Machine Interface Technology (NPIC\&HMIT 2006). November 12-16, 2006, Albuquerque, New Mexico. American Nuclear Society, LaGrange Park, Illinois.

Hashemian H, CJ Kiger, GW Morton and BD Shumaker. 2011. "Wireless Sensor Applications in Nuclear Power Plants." Nuclear Technology 173(1):8-16.

Hashemian HM. 2011b. "Oconee: the USA's First Fully Digital Nuclear Power Station." Modern Power Systems 31(1):73-75.

Hashemian HM. 2011c. "On-line Monitoring Applications in Nuclear Power Plants." Progress in Nuclear Energy 53(2):167-181.

Hashemian HM and WC Bean. 2011. "Advanced Cable-Testing Techniques for Nuclear Power Plants." Nuclear Technology 176(3):414-429.

Hashemian HM, GW Morton, BD Shumaker, D Lillis and S Orme. 2004. "Calibration Reduction System Implementation at the Sizewell B Nuclear Power Plant." In 4th International Topical Meeting on Nuclear 
Plant Instrumentation, Control and Human Machine Interface Technology, pp. 1227-1233. September 19-22, 2004, Columbus, Ohio. American Nuclear Society.

Haupt RL. 1995. "An Intriduction to Genetic Algorithms for Electromagnetics." IEEE Antennas and Propagation Magazine 37:7-15.

Haynes HD. 1990. "Evaluation of Check Valve Monitoring Methods." In Proceedings of the U.S. Nuclear Regulatory Commission, Seventeenth Water Reactor Safety Information Meeting pp. 39-58. October 23-25, 1989, Rockville, Maryland. U.S. Nuclear Regulatory Commission, Washington, D.C. NUREG/CP-0105.

Heathcote MJ. 1998. J\&P Transformer Book, Twelfth Edition. Elsevier Science Ltd., Oxford, United Kingdom.

Hecht H. 2006. "Prognostics for Electronic Equipment: An Economic Perspective." In 2006 Annual Reliability and Maintainability Symposium (RAMS '06), pp. 165-168. January 23-26, 2006, Newport Beach, California. IEEE, Piscataway, New Jersey.

Heo GY. 2008. "Condition Monitoring Using Empirical Models: Technical Review and Prospects for Nuclear Applications." Nuclear Engineering and Technology 40(1):49-68.

Hess A, G Calvello and T Dabney. 2004. "PHM a Key Enabler for the JSF Autonomic Logistics Support Concept." In 2004 IEEE Aerospace Conference, pp. 3543-3549. March 6-13, 2004, Big Sky, Montana. IEEE, Piscataway, New Jersey.

Hess A, G Calvello and P Frith. 2005. "Challenges, Issues, and Lessons Learned Chasing the "Big P": Real Predictive Prognostics. Part 1." In 2005 IEEE Aerospace Conference, pp. 3610-3619. March 5-12, 2005, Big Sky, Montana. IEEE, Piscataway, New Jersey. Paper \#1595.

Hess A and L Fila. 2002. "The Joint Strike Fighter (JSF) PHM Concept: Potential Impact on Aging Aircraft Problems." In 2002 IEEE Aerospace Conference, pp. 3021-3026 Vol. 6. March 9-16, 2002, Big Sky, Montana. IEEE, Piscataway, New Jersey.

Hines JW and E Davis. 2005. "Lessons Learned from the US Nuclear Power Plant On-Line Monitoring Programs." Progress in Nuclear Energy 46(3-4):176-189.

Hines JW, D Garvey, R Seibert and A Usynin. 2008a. Technical Review of On-line Monitoring Techniques for Performance Assessment, Volume 2: Theoretical Issues. NUREG/CR-6895, Vol. 2, U.S. Nuclear Regulatory Commission, Washington, D.C.

Hines JW, J Garvey, J Preston and A Usynin. 2008b. "Tutorial: Empirical Methods for Process and Equipment Prognostics." In 53rd Annual Reliability and Maintainability Symposium (RAMS), 2008 Proceedings. January 28-31, 2008, Las Vegas, Nevada.

Hines JW, J Garvey, R Seibert and A Usynin. 2008c. Technical Review of On-line Monitoring Techniques for Performance Assessment, Volume 3: Limiting Case Studies. NUREG/CR-6895, Vol. 3, U.S. Nuclear Regulatory Commission, Washington, D.C.

Hines JW and R Seibert. 2006. Technical Review of On-Line Monitoring Techniques for Performance Assessment: State-of-the-Art. NUREG/CR-6895, Volume 1, U.S. Nuclear Regulatory Commission, Washington, D.C. 
Hines JW, BR Upadhyaya, JM Doster, RM Edwards, KD Lewis, P Turinsky and JB Coble. 2011. Advanced Instrumentation and Control Methods for Small and Medium Reactors with IRIS Demonstration. Report No. DE-FG07-07ID14895/UTNE/2011-3, The University of Tennessee, Knoxville, Tennessee.

Hines JW and A Usynin. 2005. "MSET Performance Optimization Through Regularization." Nuclear Engineering and Technology 32(2):177-184.

Hines JW and A Usynin. 2008. "Current Computational Trends in Equipment Prognostics." International Journal of Computational Intelligence Systems 1(1):95-109.

Hines JW, A Usynin and A Urmanov. 2006. "Prognosis of Remaining Useful Life for Complex Engineering Systems." In 5th International Topical Meeting on Nuclear Plant Instrumentation Controls and Human Machine Interface Technology (NPIC\&HMIT 2006). November 12-16, 2006, Albuquerque, New Mexico. American Nuclear Society, LaGrange Park, Illinois.

Hines JW, DJ Wrest and RE Uhrig. 1996. "Plant Wide Sensor Calibration Monitoring." In Proceedings of the 1996 IEEE International Symposium on Intelligent Control, pp. 378-383. September 15-18, 1996, Dearborn, Michigan. IEEE, Piscataway, New Jersey.

Ho Y-C and DL Pepyne. 2001. "Simple Explanation of the No Free Lunch Theorem of Optimization." In Proceedings of the 40th IEEE Conference on Decision and Control, pp. 4409-4414 Vol. 5. December 4-7, 2001, Orlando, Florida. IEEE, Piscataway, New Jersey.

Hofmeister JP, J Judkins, E Ortiz, DL Goodman and P Lall. 2006a. Real-Time BIST Detector for BGA Faults in Field Programmable Gate Arrays (FPGAs). Ridgetop Group, Inc., Tucson, Arizona. Available at http://www.ridgetopgroup.com/doc/WP-RT_BIST_Detector-07.pdf.

Hofmeister JP, P Lall and R Graves. 2006b. "In-Situ, Real-Time Detector for Fauls in Solder Joint Networks Belonging to Operational, Fully Programmed Field Programmable Gate Arrays (FPGAs)." In AUTOTESTCON 2006 - IEEE Systems Readiness Technology Conference, pp. 237-243. September 1821, 2006, Anaheim, California. IEEE, Piscataway, New Jersey.

Hoole S, S Subramaniam, R Saldanha, J Coulomb and J Sabonnadiere. 1991. "Inverse Problem Methodology and Finite Elements in the Identification of Cracks, Sources, Materials and Their Geometry in Inaccessible Locations." IEEE Transactions on Magnetics 27(3):3433-3443.

Howard P. 2005. "Prognostic Technology - New Challenges." In Proceedings of the 59th Machinery Failure Prevention Technology (MFPT) Conference, pp. 3-8. April 18-21, 2005, Virginia Beach, Virginia. Society Machinery Failure Prevention Technology.

Howlader MK, PD Ewing and J Dion. 2010. "Issues Associated with Deploying Wireless Systems in Nuclear Facilities." In 7th International Topical Meeting on Nuclear Plant Instrumentation, Control, and Human-Machine Interface Technologies (NPIC\&HMIT 2010), pp. 1510-1521, Vol. 3. November 7-11, 2010, Las Vegas, Nevada. American Nuclear Society, LaGrange Park, Illinois.

Hundhausen RJ. 2004. "Loads, Damage Identification and NDE/SHM Data Fusion in Parasitic Thermal Protection Systems Using Passive Vibration-Based Methods." In Proceedings of the 2nd European Workshop on Structural Health Monitoring. July 7-9, 2004, Munich, Germany. 
Hutton PH. 1993. "Listening to Reactor Pressure Boundaries for the Sounds of Cracks and Leaks." Nuclear Engineering International 38(47):38-40.

Hutton PH, JF Dawson, MA Friesel, JC Harris and RA Pappas. 1984. Acoustic Emission Monitoring of Hot Functional Testing: Watts Bar Unit 1 Nuclear Reactor. NUREG/CR-3693, U.S. Nuclear Regulatory Commission, Washington, D.C.

Hutton PH, MA Friesel and JF Dawson. 1993. Continuous AE Crack Monitoring of a Dissimilar Metal Weldment at Limerick Unit 1. NUREG/CR-5963, PNL-8844, U.S. Nuclear Regulatory Commission, Washington, DC.

Hutton PH, RJ Kurtz, MA Friesel, JR Skorpik and JF Dawson. 1991. Acoustic Emission/Flaw Relationships for In-Service Monitoring of LWRs. NUREG/CR-5645, PNL-7479, U.S. Nuclear Regulatory Commission, Washington, D.C.

Hwang K, W Lord, S Mandayam, L Udpa and S Udpa. 1997. "A Multiresolution Approach for Characterizing MFL Signatures from Gas Transmisson Pipeline Inspections." In Review of Progress in Quantitative Nondestructive Evaluation, Volume 16, pp. 733-739. August 3-8, 1997, San Diego, California. Plenum Press, New York.

Hyers RW, JG McGowan, KL Sullivan, JF Manwell and BC Syrett. 2006. "Condition Monitoring and Prognosis of Utility Scale Wind Turbines." Energy Materials 1(3):187-203.

IAEA. 1997. Pilot Study on the Management of Ageing of Instrumentation and Control Cables - Results of a Coordinated Research Program (1993-1995). IAEA-TECDOC-932, International Atomic Energy Agency, Vienna, Austria.

IAEA. 2000. Assessment and Management of Ageing of Major Nuclear Power Plant Components Important to Safety: In-containment Instrumentation and Control Cables. Volume I. IAEA-TECDOC1188, International Atomic Energy Agency, Vienna, Austria.

IAEA. 2002. Guidebook on Non-destructive Testing of Concrete Structures, Training Course Series No. 17. IAEA-TCS-17, International Atomic Energy Agency, Vienna, Austria.

IAEA. 2005. Assessment and Management of Ageing of Major Nuclear Power Plant Components Important to Safety: BWR Pressure Vessel Internals. IAEA-TECDOC-1471, International Atomic Energy Agency (IAEA), Vienna, Austria.

IAEA. 2007. Assessment and Management of Ageing of Major Nuclear Power Plant Components Important to Safety: PWR Pressure Vessel Internals, 2007 Update. IAEA-TECDOC-1557, International Atomic Energy Agency (IAEA), Vienna, Austria.

IAEA. 2008a. On-line Monitoring for Improving Performance of Nuclear Power Plants - Part 1: Instrument Channel Monitoring. IAEA Nuclear Energy Series NP-T-1.1, International Atomic Energy Agency (IAEA), Vienna, Austria.

IAEA. 2008b. On-line Monitoring for Improving Performance of Nuclear Power Plants - Part 2: Process and Component Condition Monitoring and Diagnostics. IAEA Nuclear Energy Series NP-T-1.2, International Atomic Energy Agency (IAEA), Vienna, Austria. 
IAEA. 2010. Risk-informed In-service Inspection of Piping Systems of Nuclear Power Plants: Process, Status, Issues and Development. Nuclear Energy Series NP-T-3.1, International Atomic Energy Agency (IAEA), Vienna, Austria.

IAEA. 2012. Advanced Surveillance Diagnostics, and Prognostics Techniques Used for Health Monitoring of Systems, Structures, and Components in Nuclear Power Plants: CRP Report Volume I. IAEA-NE-D-NP-T-3.14, International Atomic Energy Agency (IAEA), Vienna, Austria. In press.

IEC. 2010. Nuclear Power Plants. Instrumentation and Control Important to Safety. Management of Ageing of Electrical Cabling Systems. IEC 62465, International Electrotechnical Commission (IEC), Geneva, Switzerland.

IEEE. P1856-Standard Framework for Prognostics and Health Management of Electronic Systems. Accessed June 25, 2012. Available at http://standards.ieee.org/develop/project/1856.html. Under development.

Illi OJ, FL Greitzer, LJ Kangas and T Reeve. 1994. "Artificial Neural Network System for Diagnosing Gas Turbine Engine Fuel Faults." In Mechanical Failures Prevention Group Conference. April 19-21, 1994, Wakefield, Massachusetts. Department of Energy, Washington, D.C. PNL-SA-22914; CONF9404179-1.

Ingersoll DT. 2009. "Deliberately Small Reactors and the Second Nuclear Era." Progress in Nuclear Energy 51:589-603.

Inman DI, CR Farrar, V Lopes and V Steffen, Eds. 2005. Damage Prognosis. Wiley, Chichester, West Sussex, England.

Isermann R. 1984. "Process Fault Detection Based on Modeling and Estimation Methods-A Survey." Automatica 20(4):387-404.

ISO. 2003. Condition Monitoring and Diagnostics of Machines - Data Processing, Communication and Presentation - Part 1: General Guidelines. ISO 13374-1:2003, International Organization for Standardization (ISO), Geneva, Switzerland.

ISO. 2004. Condition Monitoring and Diagnostics of Machines - Prognostics - Part 1: General Guidelines. ISO 13381-1:2004, International Organization for Standardization (ISO), Geneva, Switzerland.

ISO. 2007. Condition Monitoring and Diagnostics of Machines - Data Processing, Communication and Presentation - Part 2: Data Processing. ISO 13374-2:2007, International Organization for Standardization (ISO), Geneva, Switzerland.

ISO. 2009. Industrial Automation Systems and Integration - Diagnostics, Capability Assessment and Maintenance Applications Integration - Part 1: Overview and General Requirements. ISO 184351:2009, International Organization for Standardization (ISO), Geneva, Switzerland.

ISO. 2012. Condition Monitoring and Diagnostics of Machines - Data Processing, Communication and Presentation - Part 3: Communications. ISO 13374-3, International Organization for Standardization (ISO), Geneva, Switzerland. 
Jardine AKS and AHC Tsang. 2006. Maintenance, Replacement, and Reliability: Theory and Applications. CRC Press, Boca Raton, Florida.

Jarrell DB, DR Sisk and LJ Bond. 2004. "Prognostics and Conditioned-Based Maintenance: A New Approach to Precursive Metrics." Nuclear Technology 145(3):275-286.

Jaw LC and W Merrill. 2008. "CBM+ Research Environment - Facilitating Technology Development, Experimentation, and Maturation." In 2008 IEEE Aerospace Conference, pp. 1-6. March 1-8, 2008, Big Sky, Montana. IEEE, Piscataway, New Jersey.

Jenkins LS. 1985. "Cracked Shaft Detection on Large Vertical Nuclear Reactor Coolant Pump." In Instability in Rotating Machinery, pp. 253-265. June 10-14, 1985, Carson City, Nevada. NASA, Scientific \& Technical Information Branch. NASA Conference Publication No. 2409.

Joshi A. 2006. Inverse Problems in the Non-destructive Evaluation of Gas Transmission Pipelines Using Magnetic Flux Leakage. Ph.D. Thesis, Michigan State University, East Lansing, Michigan.

Judd MD, L Yang, CJ Bennoch and IBB Hunter. 2004. "UHF Diagnostic Monitoring Techniques for Power Transformers." Presented at EPRI Substation Equipment Diagnostics Conference XII, New Orleans, February 15-18, 2004. Available at http://www.marubun.jp/product/measurement/electric/qgc18e000000isvo-att/dms_2.pdf.

Jung JC and PH Seong. 2006. "An Improved Method for Reactor Coolant Pump Abnormality Monitoring Using Power Line Signal Analysis." Nuclear Engineering and Design 236(1):57-62.

Kacprzynski GJ, MJ Roemer and AJ Hess. 2002. "Health Management System Design: Development, Simulation and Cost/Benefit Optimization." In 2002 IEEE Aerospace Conference, pp. 3065-3072 Vol. 6. March 9-16, 2002, Big Sky, Montana. IEEE, Piscataway, New Jersey.

Kacprzynski GJ, A Sarlashkar, MJ Roemer, A Hess and B Hardman. 2004. "Predicting Remaining Life by Fusing the Physics of Failure Modeling with Diagnostics." Journal of the Minerals, Metals and Materials Society 56(3):29-35.

Kafka P. 2002. "Reflections on the Status of Risk Management in Nuclear Technology." In Proceedings of the Joint ESA-NASA Space-Flight Safety Conference, pp. 403-410. June 11-14, 2002, Noordwijk, Netherlands. European Space Agency.

Kalgren PW, M Baybutt, A Ginart, C Minnella, MJ Roemer and T Dabney. 2007. "Application of Prognostic Health Management in Digital Electronic Systems." In 2007 IEEE Aerospace Conference, pp. 1-9. March 3-10, 2007, Big Sky, Montana. IEEE, Piscataway, New Jersey.

Kangas LJ, FL Greitzer and OJ Illi. 1994. "TEDANN: Turbine Engine Diagnostic Artificial Neural Network." In Advanced Information Systems and Technology Conference. March 28-30, 1994, Williamsburg, Virginia. U.S. Department of Energy, Washington, D.C. PNL-SA-24733; CONF9403233-1.

Katipamula S and MR Brambley. 2005a. "Methods for Fault Detection, Diagnostics, and Prognostics for Building Systems-A Review, Part I." HVAC\&R Research 11(1):3-25.

Katipamula S and MR Brambley. 2005b. "Methods for Fault Detection, Diagnostics, and Prognostics for Building Systems_A Review, Part II." HVAC\&R Research 11(2):169-187. 
Kelkar N, A Dasgupta, M Pecht, I Knowles, M Hawley and D Jennings. 1997. "'Smart' Electronic Systems for Condition-Based Health Management." Quality and Reliability Engineering International 13(1):3-8.

Keller K, K Swearingen, J Sheahan, M Baily, J Dunsdon, B Cleeve, KW Przytula and B Jordan. 2006. "Aircraft Electrical Power Systems Prognostics and Health Management." In 2006 IEEE Aerospace Conference. March 4-11, 2006, Big Sky, Montana. IEEE, Piscataway, New Jersey.

Khaleel MA and FA Simonen. 2009. Evaluations of Structural Failure Probabilities and Candidate Inservice Inspection Programs. NUREG/CR-6986, PNNL-13810, U.S. Nuclear Regulatory Commission, Washington, D.C.

Kharoufeh JP and SM Cox. 2005. "Stochastic Models for Degradaton-Based Reliability." IIE Transactions 37:533-542.

Kiger C. 2010. "The Emerging Role of Wireless Technologies in Nuclear Power Plants." In 7th International Topical Meeting on Nuclear Plant Instrumentation, Control, and Human-Machine Interface Technologies (NPIC\&HMIT 2010), pp. 1498-1509, Vol. 3. November 7-11, 2010, Las Vegas, Nevada. American Nuclear Society, LaGrange Park, Illinois.

Kim J, P Ramuhalli, L Udpa and S Udpa. 2006. "Automated Multichannel Signal Classification Systems for Ultrasonic Nondestructive Evaluation." Key Engineering Materials 321-323:1266-1269.

Kim Y, L Jofre, FD Flaviis and MQ Feng. 2003. "Microwave Reflection Tomographic Array for Damage Detection of Civil Structures." IEEE Transactions on Antennas and Propagation 51(11):30223032.

Kirby M. 2012. "Comprehensive Approach to Buried Pipe Examinations." In 9th International Conference on NDE in Relation to Structural Integrity for Nuclear and Pressurized Components. May 22-24, 2012, Bellevue, Washington.

Kirkpatrick S, CD Gelatt and MP Vecchi. 1983. "Optimization by Simulated Annealing." Science 220(4598):671-680.

Koo IS and WW Kim. 2000. "The Development of Reactor Coolant Pump Vibration Monitoring and a Diagnostic System in the Nuclear Power Plant." ISA Transactions 39(3):309-316.

Koppen M. 2004. "No-Free-Lunch Theorems and the Diversity of Algorithms." In Congress on Evolutionary Computation (CEC2004), pp. 235-241 Vol. 1. June 19-23, 2004, Portland, Oregon.

Kothamasu R, SH Huang and WH VerDuin. 2006. "System Health Monitoring and Prognostics - A Review of Current Paradigms and Practices." The International Journal of Advanced Manufacturing Technology 28(9-10):1012-1024.

Kulkarni SS and JD Achenbach. 2008. "Structural Health Monitoring and Damage Prognosis in Fatigue." Structural Health Monitoring 7(1):37-49.

Kumar D and B Klefsjö. 1994. "Proportional Hazards Model: A Review." Reliability Engineering \& System Safety 44(2):177-188. 
Kumar M and P Ramuhalli. 2005a. "Dynamic Programming Based Multichannel Image Restoration." In 2005 IEEE International Conference on Acoustics, Speech, and Signal Processing (ICASSP '05), pp. 609612. March 18-23, 2005, Philadelphia, Pennsylvania. IEEE, Piscataway, New Jersey.

Kumar M and P Ramuhalli. 2005b. "Maximum Likelihood Wavelet Fusion for Aerospace NDE Applications." In 2005 IEEE International Conference on Electro Information Technology. May 22-25, 2005, Lincoln, Nebraska. IEEE, Piscataway, New Jersey.

Kumar M, P Ramuhalli and Z Liu. 2005. "Multiresolution Data Fusion Algorithms for Aerospace Applications." In Review of Progress in Quantitative Nondestructive Evaluation, Volume 24, pp. 720727. July 25-30, 2004, Golden, Colorado. American Institute of Physics, Melville, New York.

Kunerth DC and T McJunkin. 2011. "Nondestructive Evaluation of Nuclear Grade Graphite." In 38th Annual Review of Progress in QNDE. July 17-22, 2011, Burlington, Vermont.

Kusiak A and W Li. 2011. "The Prediction and Diagnosis of Wind Turbine Faults." Renewable Energy 36:16-23.

Lall P, R Lowe and K Goebel. 2011. "Prognostics and Health Monitoring of Electronic Systems." In 2011 12th International Conference on Thermal, Mechanical and Multi-Physics Simulation and Experiments in Microelectronics and Microsystems (EuroSimE 2011), pp. 1/17-7/17. April 18-20, 2011, Linz, Austria. IEEE Computer Society, Piscataway, New Jersey.

Lall P, M Pecht and E Harkim. 1997. Influence of Temperature on Microelectronics and System Reliability. CRC Press, New York.

Lapworth JA, PN Jarman and IR Funnell. 1995. "Condition Assessment Techniques for Large Power Transformers." In Proceedings Second International Conference on the Reliability of Transmission and Distribution Equipment (Conf. Publ. No.406), pp. 85-90. March 29-31, 1995, Coventry, United Kingdom. Institution of Electrical Engineers (IEE), Stevenage, United Kingdom.

Leao BP, KT Fitzgibbon, LC Puttini and GPB de Melo. 2008a. "Cost-Benefit Analysis Methodology for PHM Applied to Legacy Commercial Aircraft." In 2008 IEEE Aerospace Conference, pp. 1-13. March 1-8, 2008, Big Sky, Montana. IEEE, Piscataway, New Jersey.

Leao BP, JPP Gomes, RKH Galvao and T Yoneyama. 2010. "How to Tell the Good from the Bad in Failure Prognostics Methods." In 2010 IEEE Aerospace Conference, p. 7 pp. March 6-13, 2010, Big Sky, Montana. IEEE, Piscataway, New Jersey.

Leao BP, JPP Gomes and T Yoneyama. 2011. "Improvements on the Offline Performance Evaluation of Fault Prognostics Methods." In 2011 IEEE Aerospace Conference. March 5-12, 2011, Big Sky, Montana. IEEE, Piscataway, New Jersey.

Leao BP, T Yoneyama, GC Rocha and KT Fitzgibbon. 2008b. "Prognostics Performance Metrics and Their Relation to Requirements, Design, Verification and Cost-Benefit." In 1st International Conference on Prognostics and Health Management (PHM 2008). October 6-9, 2008, Denver, Colorado. IEEE, Piscataway, New Jersey.

Lee J-H, M-R Lee, J-T Kim, V Luk and Y-H Jung. 2006. "A Study of the Characteristics of the Acoustic Emission Signals for Condition Monitoring of Check Valves in Nuclear Power Plants." Nuclear Engineering and Design 236(13):1411-1421. 
Lee S and P Bajcsy. 2004. "Multisensor Raster and Vector Data Fusion Based on Uncertainty Modeling." In 2004 International Conference on Image Processing (ICIP 2004), pp. 3355-3358. October 18-21, 2004, Singapore. IEEE, Piscataway, New Jersey.

Legendre S, D Massicotte, J Goyette and TK Bose. 2000. "Wavelet-Transform-Based Method of Analysis for Lamb-Wave Ultrasonic NDE Signals." IEEE Transactions on Instrumentation and Measurement 49(3):524-530.

Li F and BR Upadhyaya. 2011. "Design of Sensor Placement for an Integral Pressurized Water Reactor Using Fault Diagnostic Observability and Reliability Criteria." Nuclear Technology 173(1):17-25.

Li Y, G Liu, B Shanker, Y Sun, P Sacks, L Udpa and S Udpa. 2001. "An Adjoint Equation Based Method for 3D Eddy Current NDE Signal Inversion." In Electromagnetic Nondestructive Evaluation (V), pp. 89-96. June 28-30, 2001, Budapest, Hungary. IOS Press, Amsterdam.

Liang T, J DeCastro, G Kacprzynski, K Goebel and G Vachtsevanos. 2010a. "Filtering and Prediction Techniques for Model-based Prognosis and Uncertainty Management." In 2010 Prognostics and System Health Management Conference (PHM '10). January 12-14, 2010, Macao. IEEE, Piscataway, New Jersey.

Liang T, GJ Kacprzynski, JR Bock and M Begin. 2006. "An Intelligent Agent-Based Self-Evolving Maintenance and Operations Reasoning System." In 2006 IEEE Aerospace Conference. March 4-11, 2006, Big Sky, Montana. IEEE, Piscataway, New Jersey.

Liang T, GJ Kacprzynski, K Goebel, A Saxena, B Saha and G Vachtsevanos. 2008. 1st International Conference on Prognostics and Health Management (PHM 2008), pp. 1-9. October 6-9, 2008, Denver, Colorado. IEEE, Piscataway, New Jersey.

Liang T, GJ Kacprzynski, K Goebel and G Vachtsevanos. 2009. "Methodologies for Uncertainty Management in Prognostics." In 2009 IEEE Aerospace Conference, pp. 1-12. March 7-14, 2009, Big Sky, Montana. IEEE, Piscataway, New Jersey.

Liang T, GJ Kacprzynski, K Goebel and G Vachtsevanos. 2010b. "Case Studies for PrognosticsEnhanced Automated Contingency Management for Aircraft Systems." In 2010 IEEE Aerospace Conference, pp. 1-11. March 6-13, 2010, Big Sky, Montana. IEEE, Piscataway, New Jersey.

Liang T, ME Orchard, K Goebel and G Vachtsevanos. 2011. "Novel Metrics and Methodologies for the Verification and Validation of Prognostic Algorithms." In 2011 IEEE Aerospace Conference, pp. 1-8. March 5-12, 2011, Big Sky, Montana. IEEE, Piscataway, New Jersey.

Liao H, W Zhao and H Guo. 2006. "Predicting Remaining Useful Life of an Individual Unit Using Proportional Hazards Model and Logistic Regression Model." In 2006 Annual Reliability and Maintainability Symposium (RAMS '06), pp. 127-132. January 23-26, 2006, Newport Beach, California. IEEE, Piscataway, New Jersey.

Lillis D. 2010. "Use of Online Monitoring to Support Condition Based Maintenance of Safety Category Sensors at Sizewell 'B' Nuclear Power Plant." In 7th International Topical Meeting on Nuclear Plant Instrumentation, Control, and Human-Machine Interface Technologies (NPIC\&HMIT 2010), pp. 856874, Vol. 2. November 7-11, 2010, Las Vegas, Nevada. American Nuclear Society, LaGrange Park, Illinois. 
Lillis D and S Orme. 2005. "Implementation of On-Line Monitoring for Transmitter Calibration Period Extension at Sizewell B." In IAEA Technical Meeting on On-Line Condition Monitoring of Equipment and Processes in NPPs Using Advanced Diagnostic Systems. June 27-30, 2005, Knoxville, Tennessee.

Lindberg J. 2011. "Monitoring and NDE to Support Long Term Operation of the Nuclear Fleet Augmented Containment Inspection." In 38th Annual Review of Progress in QNDE. July 17-22, 2011, Burlington, Vermont.

Line JK and NS Clements. 2005. "A Systematic Approach for Developing Prognostic Algorithms on Large Complex Systems." In 2005 IEEE Aerospace Conference, pp. 1-7. March 5-12, 2005, Big Sky, Montana. IEEE, Piscataway, New Jersey.

Line JK and NS Clements. 2006. "Prognostics Usefulness Criteria." In 2006 IEEE Aerospace Conference. March 4-11, 2006, Big Sky, Montana. IEEE, Piscataway, New Jersey.

Line JK, L Wenying, M King and RG Tryon. 2007. "Prognostics and Maintenance Modeling for Electronic Components." In AUTOTESTCON 2007 - Systems Readiness Technology Conference, pp. 639-645. September 17-20, 2007, Baltimore, Maryland. IEEE, Piscataway, New Jersey.

Liu H, J Yu, P Zhang and X Li. 2011. "Review on Verification and Validation Technology in Integrated Health Management System." In 10th International Conference on Electronic Measurement \& Instruments (ICEMI 2011), pp. 125-129. August 16-19, 2011, Chengdu, China. DOI 10.1109/icemi.2011.6037780. IEEE, Piscataway, New Jersey.

Liu J, A Saxena, K Goebel, B Saha and W Wang. 2010. "An Adaptive Recurrent Neural Network for Remaining Useful Life Prediction of Lithium-Ion Batteries." In Annual Conference of the Prognostics and Health Management Society, 2010. October 13-16, 2010, Portland, Oregon.

Liu WC, PT Kuo and SS Lee. 1997. Aging Management of Nuclear Power Plant Containments for License Renewal. NUREG-1611, U.S. Nuclear Regulatory Commission, Washington, D.C.

Liu Z, P Ramuhalli, S Safizadeh and DS Forsyth. 2008. "Combining Multiple Nondestructive Inspection Images with a Generalized Additive Model." Measurement Science and Technology 19(8):085701.

Liu Z, MS Safizadeh, DS Forsyth and BA Lepine. 2003. "Data Fusion Method for the Optimal Mixing of Multi-Frequency Eddy Current Signals." In Review of Progress in Quantitative Nondestructive Evaluation, Vol. 22, pp. 577-584. July 14-19, 2002, Bellingham, Washington. American Institute of Physics, Melville, New York.

Lois A and M Ruch. 2006. "Assessment of Martensite Content in Austenitic Stainless Steel Specimens by Eddy Current Testing." Insight 48(1):26-29.

Lord W, YS Sun, SS Udpa and S Nath. 1988. "A Finite Element Study of the Remote Field Eddy Current Phenomenon." IEEE Transactions on Magnetics 24(1):435-438.

Luk KH. 1993a. Boiling-Water Reactor Internals Aging Degradation Study. NUREG/CR-5754, U.S. Nuclear Regulatory Commission, Washington, D.C.

Luk KH. 1993b. Pressurized-Water Reactor Internals Aging Degradation Study. NUREG/CR-6048, U.S. Nuclear Regulatory Commission, Washington, D.C. 
Lybeck N, B Pham, M Tawfik, JB Coble, RM Meyer, P Ramuhalli and LJ Bond. 2011. Lifecycle Prognostics Architecture for Selected High Cost Active Components. INL/EXT-11-22915, Idaho National Laboratory, Idaho Falls, Idaho.

Ma J and J Jiang. 2011. "Applications of Fault Detection and Diagnosis Methods in Nuclear Power Plants: A Review." Progress in Nuclear Energy 53(3):255-266.

Malhotra VM and NJ Carino. 2004. Handbook on Nondestructive Testing of Concrete. CRC Press, LLC, West Conshohocken, Pennsylvania. ISBN 0-8031-2099-0.

Maren AJ, RM Pap and CT Harston. 1989. "A Hierarchical Data Structure Representation for Fusion of Multisensor Information." In Proceedings SPIE Technical Symposia on Aerospace Sensing, Sensor Fusion Section, pp. 162-178. March 28-29, 1989, Orlando, Florida. The International Society for Optical Engineering (SPIE), Bellingham, Washington.

Mathew S, D Das, R Rossenberger and M Pecht. 2008. "Failure Mechanisms Based Prognostics." In 1st International Conference on Prognostics and Health Management (PHM 2008). October 6-9, 2008, Denver, Colorado. IEEE, Piscataway, New Jersey.

Matlack KH, JJ Wall, J-Y Kim, J Qu, LJ Jacobs and H-W Viehrig. 2012. "Evaluation of Radiation Damage Using Nonlinear Ultrasound." Journal of Applied Physics 111(5):054911-054913.

McArthur S, S Strachan and G Jahn. 2004. "The Design of a Multi-Agent Transformer Condition Monitoring System." IEEE Transactions on Power Systems 19(4):1845-1852.

McShane JL and NH Ulerich. 1992. "System and Method for Valve Monitoring Using Pipe-Mounted Ultrasonic Transducers." U.S. Patent 5,115,672.

Medill RB. 2003. SmartSignal Cuts Travel Delays by Reducing Surprise Repairs. Goliath Business Knowledge on Demand. Accessed March 12, 2012. Available at http://goliath.ecnext.com/coms2/gi 0199-2673310/SmartSignal-cuts-travel-delays-by.html.

Meyer RM, LJ Bond and P Ramuhalli. 2012a. "Online Condition Monitoring to Enable Extended Operation of Nuclear Power Plants." Nuclear Safety and Simulation 3(1):31-50.

Meyer RM, LJ Bond, P Ramuhalli and SR Doctor. 2010. Advanced Instrumentation, Information, and Control System Technologies: Nondestructive Examination Technologies - NDE Technology for the Inservice Inspection of Commercial Nuclear Power Plants: An Assessment PNNL-19705, Pacific Northwest National Laboratory, Richland, Washington.

Meyer RM, JB Coble, P Ramuhalli and LJ Bond. 2011a. Advanced Instrumentation, Information, and Control System Technologies: Nondestructive Examination Technologies - FY11 Report. PNNL-20671, Pacific Northwest National Laboratory, Richland, Washington.

Meyer RM, SE Cumblidge, P Ramuhalli, BE Watson, SR Doctor and LJ Bond. 2011b. "Acoustic Emission and Guided Wave Monitoring of Fatigue Crack Growth on a Full Scale Pipe Specimen." In Proceedings of SPIE, Volume 7984 - Health Monitoring of Structural and Biological Systems 2011; SPIE Smart Structures/NDE. March 6-10, 2011, San Diego, California. Society of Photo-Optical Instrumentation Engineers, Bellingham, Washington. 
Meyer RM, P Ramuhalli, BG Braatz and SR Doctor. 2012b. Application of Acoustic Emission and Other Online Monitoring Technologies to High Temperature Gas Reactors. PNNL-20606, Pacific Northwest National Laboratory, Richland, Washington.

Miljkovic D. 2011. "Fault Detection Methods: A Literature Survey." In MIPRO, 2011 Proceedings of the 34th International Convention, pp. 750-755. May 23-27, 2011, Opatija, Croatia. IEEE, Piscataway, New Jersey.

Millar RC. 2009. "Defining Requirements for Advanced PHM Technologies for Optimal Reliability Centered Maintenance." In 2009 IEEE Aerospace Conference, pp. 1-7. March 7-14, 2009, Big Sky, Montana. IEEE, Piscataway, New Jersey.

Miller C. 2008. Nondestructive Evaluation: A Review of NDE Performance Demonstrations - NDE Round Robin Report. Report No. 1016969, Electric Power Research Institute, Palo Alto, California.

Milne R. 1987. "Strategies for Diagnosis." IEEE Transactions on Systems, Man and Cybernetics 17(3):333-339.

Miralles R, L Vergara and J Gosalbez. 2004. "Material Grain Noise Analysis by Using Higher-Order Statistics." IEEE Transactions on Signal Processing 84(1):197-205.

Mishra K and KC Gross. 2003. "Dynamic Simulation Tool for Improved Performance Modeling and Resource Provisioning of Enterprise Servers." In 14th International Symposium on Software Reliability Engineering (ISSRE 2003). November 17-20, 2003, Denver, Colorado. IEEE Computer Society.

Mishra S, S Ganesan, M Pecht and J Xie. 2004. "Life Consumption Monitoring for Electronics Prognostics." In 2004 IEEE Aerospace Conference, pp. 3455-3467. March 6-13, 2004, Big Sky, Montana. IEEE, Piscataway, New Jersey.

Mishra S, M Pecht and DL Goodman. 2002. "In-situ Sensors for Product Reliability Monitoring." In Proceedings of SPIE, Volume 4755 - Design, Test, Integration, and Packaging of MEMS/MOEMS, pp. 10-19. May 6-8, 2002, Cannes, France. Society of Photo-Optical Instrumentation Engineers, Bellingham, Washington.

Monebhrrun V, B Duchene and D Lesselier. 1998. "Three-Dimensional Inversion of Eddy Current Data for Nondestructive Evaluation of Steam Generator Tubes." Inverse Problems 14:707-724.

Montgomery DC. 2005. Introduction to Statistical Quality Control. John Wiley \& Sons, Hoboken, New Jersey.

Morozov VA. 1993. Regularization Methods for Ill-Posed Problems. CRC Press.

Morris JB, DA Pommet, MA Fiddy and RV McGahan. 1995. "Imaging of Strongly Scattering Object Using a Nonlinear Filtering Technique." In Proceedings of SPIE - The International Society for Optical Engineering, pp. 27-37. July 10-11, 1995, San Diego, California. Society of Photo-Optical Instrumentation Engineers, Bellingham, Washington.

Mueller C, M Bertovic, M Pavlovic, D Kanzler, U Ewert, J Pitkänen and U Ronneteg. 2012. "Holistically Evaluating the Reliability of NDE Systems - Paradigm Shift." In 18th World Conference on Nondestructive Testing (WCNDT 2012). April 16-20, 2012, Durban, South Africa. Available at http://www.ndt.net/article/wcndt2012/papers/591 wondtfinal00590.pdf. 
Nabeshima K, T Suzudo, T Ohno and K Kudo. 2002. "Nuclear Reactor Monitoring with the Combination of Neural Network and Expert System." Mathematics and Computers in Simulation 60:233244.

Nabeshima K, T Suzudo, S Seker, E Ayaz, B Barutcu, E Turkcan, T Ohno and K Kudo. 2003. "On-line Neuro-Expert Monitoring System for Borssele Nuclear Power Plant." Progress in Nuclear Energy 43(14):397-404.

Nakamura T and M Terada. 1985. "Development of Leak Monitoring System for Pressurizer Valves." Progress in Nuclear Energy 15:175-179.

Nandhakumar N and JK Aggarwal. 1997. "Physics-Based Integration of Multiple Sensing Modalities for Scene Interpretation." Proceedings of the IEEE 85(1):147-163.

National Research Council. 1996. Mathematics and Physics of Emerging Biomedical Imaging. National Academies Press, Washington, D.C.

Naus DJ. 2007. Inspection of Nuclear Power Plant Structures - Overview of Methods and Related Applications. ORNL/TM-2007/191, Oak Ridge National Laboratory, Oak Ridge, Tennessee.

Naus DJ. 2009. "The Management of Aging in Nuclear Power Plant Concrete Structures." Journal of the Minerals Metals \& Materials Society (JOM) 61(7):35-41.

NEI. 2010. Guideline for the Management of Underground Piping and Tank Integrity. NEI 09-14, Rev. 1, Nuclear Energy Institute (NEI), Washington, D.C.

NEI. 2011. Nuclear Energy's Economic Benefits - Current and Future (White Paper). Nuclear Energy Institute, Washington, D.C. http://nei.org/filefolder/Nuclear_Energys_Economic_Benefits_Current and Future 1.pdf.

Nelson LS. 1984. "Technical Aids: The Shewhart Control Chart--Tests for Special Causes." Journal of Quality Technology 16(4):237-239.

Newhouse VL, P Karpur, JL Rose and I Amir. 1985. "A New Technique for Clutter Reduction in Ultrasonic Imaging." In Proceedings of IEEE 1985 Ultrasonics Symposium, pp. 998-1003. October 1618, 1985, San Francisco, California. IEEE, New York.

Nichols R and N McDonald. 1987. "An Introduction to the PISC II Project--Programme for the Inspection of Steel Components." British Journal of Nondestructive Testing 29(4):223-227.

Nicholson M, T Biegler and BW Brook. 2011. "How Carbon Pricing Changes the Relative Competitiveness of Low-Carbon Baseload Generating Technologies." Energy 36(1):305-313.

NIS. 2006. Borssele Nuclear Plant Definitely Staying Open Until 2033. NIS News. Netherlands. Accessed March 13, 2012. Available at http://www.nisnews.nl/public/110106 1.htm.

Norris WE, DJ Naus and HL Graves III. 1999. "Inspection of Nuclear Power Plant Containment Structures." Nuclear Engineering and Design 192:303-329. 
Novak S and M Podest. 1987. "Nuclear Power Plant Ageing and Life Extension: Safety Aspects." IAEA Bulletin:31-33. Available at http://www.iaea.org/Publications/Magazines/Bulletin/Bull294/29402043133.pdf.

NRC. 1997. Regulatory Guide 1.160, Monitoring the Effectiveness of Maintenance at Nuclear Power Plants. Rev. 2, U.S. Nuclear Regulatory Commission, Washington, D.C. Rev. 1 - January 1995.

NRC. 2000. Letter to GL Vine. "Safety Evaluation by the Office of Nuclear Reactor Regulation, Application of On-line Performance Monitoring to Extend Calibration Intervals of Instrument Channel Calibrations Required by the Technical Specifications, EPRI Topical Report (TR) 104965 'On-Line Monitoring of Instrument Channel Performance'." July 24, 2000, U.S. Nuclear Regulatory Commission, Washington, D.C. Available at http://pbadupws.nrc.gov/docs/ML0037/ML003734509.pdf.

NRC. 2001. Generic Aging Lessons Learned (GALL) Report. NUREG-1801, U.S. Nuclear Regulatory Commission, Washington, D.C.

NRC. 2005a. Generic Aging Lessons Learned (GALL) Report - Summary. NUREG-1801, Vol. 1, Rev. 1, Office of Nuclear Reactor Regulations, U.S. Nuclear Regulatory Commission, Washington, D.C.

NRC. 2005b. Generic Aging Lessons Learned (GALL) Report - Tabulation of Results. NUREG-1801, Vol. 2, Rev. 1, Office of Nuclear Reactor Regulations, U.S. Nuclear Regulatory Commission, Washington, D.C.

NRC. 2010a. Condition Monitoring Program for Electric Cables Used in Nuclear Power Plants. Draft Regulatory Guide DG-1240, U.S. Nuclear Regulatory Commission, Washington, D.C.

NRC. 2010b. Cyber Security Programs for Nuclear Facilities. Regulatory Guide 5.71, U.S. Nuclear Regulatory Commission, Washington, D.C.

NRC. 2010c. Final Report - Standard Review Plan for Review of License Renewal Applications for Nuclear Power Plants. NUREG-1800, Rev. 2, U.S. Nuclear Regulatory Commission, Washington, D.C.

NRC. 2010d. Generic Aging Lessons Learned (GALL) Report - Final Report. NUREG-1801, Rev. 2, Office of Nuclear Reactor Regulations, U.S. Nuclear Regulatory Commission, Washington, D.C.

NRC. 2011. Information Digest, 2011-2012. NUREG-1350, Vol. 23, U.S. Nuclear Regulatory Commission, Washington, D.C.

NUMARC 93-01. 1996. "Industry Guideline for Monitoring the Effectiveness of Maintenance at Nuclear Plants." Available at http://www.nrc.gov/reactors/operating/ops-experience/maintenanceeffectiveness/mainten-effect-files/numarc-93-01-rev2.pdf.

O'Donnell WJ, AB Hull and SN Malik. 2008. "Historical Context of Elevated Temperature Structural Integrity for Next Generation Plants: Regulatory Safety Issues in Structural Design Criteria of ASME Section III Subsection NH." In 2008 ASME Pressure Vessel and Piping Division Conference (PVP2008), pp. 729-738. July 27-31, 2008, Chicago, Illinois.

Oagaro JA, PJ Kulick, MT Kim, R Polikar, JC Chen and S Mandayam. 2004. A Multi-Sensor Data Fusion System for Assessing the Integrity of Gas Transmission Pipelines. Rowan University, Glassboro, New Jersey. 
Oja M, JK Line, G Krishnan and RG Tryon. 2007. "Electronic Prognostics with Analytical Models Using Existing Measurands." In Proceedings of the 61th Machinery Failure Prevention Technology (MFPT) Conference. April 17-19, 2007, Virginia Beach, Virginia. Society Machinery Failure Prevention Technology.

Orchard ME and GJ Vachtsevanos. 2007. "A Particle Filtering-Based Framework for Real-Time Fault Diagnosis and Failure Prognosis in a Turbine Engine." In 15th Mediterranean Conference on Control and Automation, pp. 1441-1446. June 27-29, 2007, Athens, Greece. IEEE, Piscataway, New Jersey.

Orsagh RF, D Brown, MJ Roemer, T Dabney and A Hess. 2005. "Prognostic Health Management for Avionics System Power Supplies." In 2005 IEEE Aerospace Conference, pp. 3585-3591. March 5-12, 2005, Big Sky, Montana. IEEE, Piscataway, New Jersey.

Orsagh RF, DW Brown, PW Kalgren, CS Byington, AJ Hess and T Dabney. 2006. "Prognostic Health Management for Avionic Systems." In 2006 IEEE Aerospace Conference. March 4-11, 2006, Big Sky, Montana. IEEE, Piscataway, New Jersey.

Park SC, YS Lee and SJ Kim. 2004. "Threshold Varying Method of Stationary Wavelet Denoising for Ultrasonic Speckle Reduction." Materials Science Forum 449-452:1153-1156.

Parks D and BR Tittmann. 2011. "Ultrasonic NDE in a Reactor Core." In 38th Annual Review of Progress in Quantitative NDE. July 17-22, 2011, Burlington, Vermont.

Pearson JC, JJ Gelfand, WE Sullivan, RM Peterson and CD Spence. 1988. "Neural Network Approach to Sensory Fusion." In Proceedings of SPIE on Sensor Fusion, pp. 103-108. Orlando, Florida. The International Society for Optical Engineering, Bellingham, Washington.

Pecht M, D Das and A Ramakrishnan. 2002. "The IEEE Standards on Reliability Program and Reliability Prediction Methods for Electronic Equipment." Microelectronic Reliability 42:1259-1266.

Pecht M and A Dasgupta. 1995. "Physics of Failure: An Approach to Reliable Product Development." Journal of the Institute of Environmental Sciences 38(5):30-34.

Pecht M, M Dube, M Natishan, R Williams, J Banner and I Knowles. 2001. "Evaluation of Built-in Test." IEEE Transactions on Aerospace and Electronic Systems 37(1):266-271.

Petrone CD, RP Correia and SC Black. 1995. Lessons Learned from Early Implementation of the Maintenance Rule at Nine Nuclear Power Plants. NUREG-1526, U.S. Nuclear Regulatory Commission, Washington, D.C.

Pipe K. 2008. "Practical Prognostics for Condition Based Maintenance." In 1st International Conference on Prognostics and Health Management (PHM 2008), pp. 1-10. October 6-9, 2008, Denver, Colorado. IEEE, Piscataway, New Jersey.

Pöckl C and W Kleinöder. 2007. "Developing and Implementation of a Fatigue Monitoring System for the New European Pressurized Water Reactor EPR." In Proceedings of the International Conference Nuclear Energy for New Europe (PORT2007), pp. 503.1-503.7. September 10-13, 2007, Portorož, Slovenia. Nuclear Society of Slovenia.

Poeckl C, S Bergholz, J Rudolph and N Wirtz. 2001. "AREVA’s Fatigue Concept (AFC) - An Integrated and Multidisciplinary Approach to the Fatigue Assessment of NPP Components." In 
International Conference on Nuclear Energy for New Europe 2010, pp. 610.1-610.13. September 6-9, 2010, Portorož, Slovenia. Nuclear Society of Slovenia. Available at http://www.djs.si/proc/port2010/pdf/610.pdf.

Polikar R, L Udpa, SS Udpa and T Taylor. 1998. "Frequency Invariant Classification of Ultrasonic Weld Inspection Signals." IEEE Transactions on Ultrasonics, Ferroelectrics and Frequency Control 45(3):614-625.

Potthast R. 2001. Point Sources and Multipoles in Inverse Scattering Theory. Chapman and Hall/CRC.

Powrie H. 2000. "Use of Electrostatic Technology for Aero Engine Oil System Monitoring." In 2000 IEEE Aerospace Conference, pp. 57-72. March 18-25, 2000, Big Sky, Montana. IEEE, Piscataway, New Jersey.

Powrie H and A Novis. 2006. "Gas Path Debris Monitoring for F-35 Joint Strike Fighter Propulsion System PHM." In 2006 IEEE Aerospace Conference. March 4-11, 2006, Big Sky, Montana. IEEE, Piscataway, New Jersey.

Qing W, S Xueqin, Y Qingxin and Y Weili. 1997. "Using Wavelet Neural Networks for the Optimal Design of Electromagnetic Devices." IEEE Transactions on Magnetics 33(2):1928-1930.

Quek ST, PS Tua and Q Wang. 2003. "Detecting Anomalies in Beams and Plate Based on the HilbertHuang Transform of Real Signals." Smart Materials and Structures 12(3):447-460.

Raj B, V Moorthy, T Jayakumar and KBS Rao. 2003. "Assessment of Microstructures and Mechanical Behaviour of Metallic Materials through Non-destructive Characterisation." International Materials Reviews 48(5):273-325.

Ramachandran KP, K Fathi and BKN Rao. 2010. "Recent Trends in Systems Performance Monitoring \& Failure Diagnosis." In IEEE International Conference on Industrial Engineering and Engineering Management (IEEM 2010), pp. 2193-2200. December 7-10, 2010, Macao. IEEE, Piscataway, New Jersey.

Ramakrishnan A and MG Pecht. 2003. "A Life Consumption Monitoring Methodology for Electronic Systems." IEEE Transactions on Components and Packaging Technologies 26(3):625-634.

Ramuhalli P. 2002. Neural Network Based Iterative Algorithms for Solving Electromagnetic NDE Inverse Problems. Ph.D. Thesis, Iowa State University, Ames, Iowa.

Ramuhalli P, LJ Bond, JW Griffin, M Dixit and CH Henager, Jr. 2010. "A Bayesian Prognostic Algorithm for Assessing Remaining Useful Life of Nuclear Power Components." In 7th International Topical Meeting on Nuclear Plant Instrumentation, Control, and Human-Machine Interface Technologies (NPIC\&HMIT 2010), pp. 875-886, Vol. 2. November 7-11, 2010, Las Vegas, Nevada. American Nuclear Society, LaGrange Park, Illinois.

Ramuhalli P and Z Liu. 2004. "Wavelet Neural Network Based Data Fusion for Improved Thickness Characterization." In Proceedings of the 30th Annual Review of Progress in Quantitative Nondestructive Evaluation, Vol. 23, pp. 589-596. July 27-August 1, 2003, Green Bay, Wisconsin. American Institute of Physics, Melville, New York. 
Ramuhalli P, L Udpa and S Udpa. 2002. "Electromagnetic NDE Signal Inversion Using Function Approximation Neural Networks." IEEE Transactions on Magnetics 38(6):3633-3642.

Ramuhalli P, L Udpa and S Udpa. 2003a. "Neural Network Based Inversion Algorithms in Magnetic Flux Leakage NDE." Journal of Applied Physics 93(10):8274-8276.

Ramuhalli P, F Yuan, U Park, J Slade and L Udpa. 2003b. "Enhancement of Magneto-Optic Images." In 9th International Workshop on Electromagnetic Nondestructive Evaluation (ENDE 2003), pp. 199-205. May 15-16, 2003, Saclay, France. IOS Press.

Rasmuson DM, TE Wierman and KJ Kvarfordt. 2005. "An Overview of the Reliability and Availability Data System (RADS)." In International Topical Meeting on Probabilistic Safety Analysis (PSA 05), pp. 388-393. September 11-15, 2005, San Francisco, California. American Nuclear Society, La Grange, Illinois.

Rasmussen B. 2005. "On-Line Monitoring for Equipment Condition Assessment: Technology Status." In IAEA Technical Meeting on On-Line Condition Monitoring of Equipment and Processes in NPPS Using Advanced Diagnostic Systems. June 27-30, 2005, Knoxville, Tennessee.

Reichard K, E Crow and T Bair. 2007. "Integrated Management of System Health in Space Applications." In 2007 Annual Reliability and Maintainability Symposium (RAMS '07), pp. 107-112. January 22-25, 2007, Orlando, Florida. IEEE, Piscataway, New Jersey.

Ren J-T, Y-W Cai, D-F Wang and X-C Xing. 2011. "A Method of Health Assessment for Aerospace Launch Site Ground Equipments." In 2011 Prognostic and Health Mangment Conference (PHM-2011) pp. 1-4. May 23-25, 2011, Shenzhen, China. IEEE, Piscataway, New Jersey. Paper MU3029.

Rich SH and V Venkatasubramanian. 1987. "Model-based Reasoning in Diagnostic Expert Systems for Chemical Process Plants." Computers \& Chemical Engineering 11(2):111-122.

Robinson DG. 2006. "Performance and Reliability Monitoring of Advanced Reactors." In 5th International Topical Meeting on Nuclear Plant Instrumentation Controls and Human Machine Interface Technology (NPIC\&HMIT 2006). November 12-16, 2006, Albuquerque, New Mexico. American Nuclear Society, LaGrange Park, Illinois.

Roemer MJ, EO Nwadiogbu and G Bloor. 2001. "Development of Diagnostic and Prognostic Technologies for Aerospace Health Management Applications." In 2001 IEEE Aerospace Conference, pp. 3139-3147 Vol. 6. March 10-17, 2001, Big Sky, Montana. IEEE, Piscataway, New Jersey.

Rose JL. 1999. Ultrasonic Waves in Solid Media. Cambridge University Press, Cambridge, United Kingdom.

Rose JL. 2002. "A Baseline and Vision of Ultrasonic Guided Wave Inspection Potential." Journal of Pressure Vessel Technology 124:273-282.

Saha B and K Goebel. 2008. "Uncertainty Management for Diagnostics and Prognostics of Batteries Using Bayesian Techniques." In 2008 IEEE Aerospace Conference. March 1-8, 2008, Big Sky, Montana. IEEE, Piscataway, New Jersey. 
Saha B, P Quach and K Goebel. 2011. "Exploring the Model Design Space for Battery Health Management." In Annual Conference of the Prognostics and Health Management Society 2011. September 25-29, 2011, Montreal, Quebec.

Sankararaman S, Y Ling, C Shantz and S Mahadevan. 2009. "Uncertainty Quantification in Fatigue Damage Prognosis." In Annual Conference of the Prognostics and Health Management Society, pp. 1-13. September 27-October 1, 2009, San Diego, California.

Saxena A, J Celaya, B Saha, S Saha and K Goebel. 2009a. "Evaluating Algorithm Performance Metrics Tailored for Prognostics." In 2009 IEEE Aerospace Conference, pp. 1-13. March 7-14, 2009, Big Sky, Montana. IEEE, Piscataway, New Jersey.

Saxena A, J Celaya, B Saha, S Saha and K Goebel. 2009b. "On Applying the Prognostics Performance Metrics." In Annual Conference of the Prognostics and Health Management Society 2009. September 27-October 1, 2009, San Diego, California.

Saxena A, J Celaya, B Saha, S Saha and K Goebel. 2010a. "Evaluating Prognostics Performance for Algorithms Incorporating Uncertainty Estimates." In 2010 IEEE Aerospace Conference. March 6-13, 2010, Big Sky, Montana. IEEE, Piscataway, New Jersey.

Saxena A, J Celaya, B Saha, S Saha and K Goebel. 2010b. Metrics for Offline Evaluation of Prognostic Performance. Report ARC-E-DAA-TN1136, Ames Research Center, National Aeronautics and Space Administration, Moffett Field, California.

Saxena A, K Goebel, D Simon and N Eklun d. 2008a. "Prognostics Challenge Competition Summary: Damage Propagation Modeling for Aircraft Engine Run-to-Failure Simulation." In 1st International Conference on Prognostics and Health Management (PHM 2008). October 6-9, 2008, Denver, Colorado. IEEE, Piscataway, New Jersey.

Saxena A, J Selaya, E Balaban, K Goebel, B Saha, S Saha and M Schwabacher. 2008b. "Metrics for Evaluating Performance of Prognostic Techniques." In 1st International Conference on Prognostics and Health Management (PHM 2008). October 6-9, 2008, Denver, Colorado. IEEE, Piscataway, New Jersey.

Schuster GJ, SR Doctor and LJ Bond. 2004. "A System for High-Resolution, Nondestructive, Ultrasonic Imaging of Weld Grains." IEEE Transactions on Instrumentation and Measurement 53(6):1526-1532.

Schwabacher M. 2005. "A Survey of Data-Driven Prognostics." In Proceedings of the AIAA Infotech@Aerospace Conference. September 26-29, 2005, Arlington, Virginia. American Institute for Aeronautics and Astronautics, Inc. Paper AIAA 2005-7002.

Schwabacher M and K Goebel. 2007. "A Survey of Artificial Intelligence for Prognostics." In Artificial Intelligence for Prognostics: Papers from the AAAI Fall Symposium, pp. 107-114. November 9-11, 2007, Arlington, Virginia. AAAI Press, Menlo Park, California. Technical Report FS-07-02.

SECY-09-0174. 2009. "Staff Progress in Evaluation of Buried Piping at Nuclear Reactor Facilities." U.S. Nuclear Regulatory Commission, Washington, D.C. NRC Policy Issue, December 2, 2009. ADAMS Accession No. ML072750402. http://pbadupws.nrc.gov/docs/ML0931/ML093160004.pdf.

Seker S, E Ayaz and E Turkcan. 2003. "Elman's Recurrent Neural Network Applications to Condition Monitoring in Nuclear Power Plant and Rotating Machinery." Enginering Applications of Artificial Intelligence 16:647-656. 
Shah VK and PE MacDonald, Eds. 1993. Aging and Life Extension of Major Light Water Reactor Components. Elsevier Science Publishers B.V., Amsterdam.

Shah VN and CJ Hookham. 1998. "Long-Term Aging of Light Water Reactor Concrete Containments." Nuclear Engineering and Design 185(1):51-81.

Shankar PM, P Karpur, VL Newhouse and JL Rose. 1989. "Split-Spectrum Processing: Analysis of Polarity Thresholding Algorithm for Improvement of Signal-to-Noise Ratio and Detectability in Ultrasonic Signals." IEEE Transactions on Ultrasonics, Ferroelectrics, and Frequency Control 36(1):101-108.

Sharp ME, JW Hines and R Austin. 2010. "Equipment Monitoring Via Transient Methods." In 7th International Topical Meeting on Nuclear Plant Instrumentation, Control, and Human-Machine Interface Technologies (NPIC\&HMIT 2010), pp. 1207-1219, Vol. 2. November 7-11, 2010, Las Vegas, Nevada. American Nuclear Society, LaGrange Park, Illinois.

Sharp ME, JW Hines and R Austin. 2011. "Advancing the Equipment Assessment Knowledge Base: Automating Prognostic Monitoring via Transient Methods." Presented at the 54th ISA POWID Symposium, June 5-10, 2011, Charlotte, North Carolina.

Sharp ME, JW Hines and R Austin. 2012. "Utilizing Available Data: Prognostic Methods via Transient Operations." Presented at MARCON 2012, February 27-March 1, 2012, Knoxville, Tennessee.

Shekhar H, R Polikar, P Ramuhalli, X Liu, M Das, L Udpa and SS Udpa. 2001. "Dynamic Thresholding for Automated Analysis and Classification of Bobbin Probe Eddy Current Data." International Journal of Applied Electromagnetics and Mechanics 15(1-4):39-46.

Sheppard JW, MA Kaufman and TJ Wilmering. 2008. "IEEE Standards for Prognostics and Health Management." In AUTOTESTCON 2008, pp. 97-103. September 8-11, 2008, Salt Lake City, Utah. IEEE, Piscataway, New Jersey.

Shin B-H, P Ramuhalli, L Udpa and S Udpa. 2004. "Independent Component Analysis for Eddy Current Inspection of Steam Generator Tubing." In Proceedings of the 30th Annual Review of Progress in Quantitative Nondestructive Evaluation, Vol. 23, pp. 597-604. July 27-August 1, 2003, Green Bay, Wisconsin. American Institute of Physics, Melville, New York.

Silverstein SD and LJ Thomas. 1993. "Analytical Comparison of Sensor Signal Processing Enhancements for NDT Synthetic Aperture Ultrasonic Imaging." IEEE Transactions on Image Processing 2(1):60-67.

Simone G and FC Morabito. 2000. "ICA-NN Based Data Fusion Approach in ECT Signal Restoration." In Proceedings of the International Joint Conference on Neural Networks (IJCNN'2000), pp. 59-64. July 24-27, 2000, Como, Italy. IEEE, Piscataway, New Jersey.

Simone G, FC Morabito, R Polikar and P Ramuhalli. 2001. "Feature Extraction Techniques for Ultrasonic Signal Classification." International Journal of Applied Electromagnetics and Mechanics 15(1-4):291-294.

Simonen FA, SR Doctor, SR Gosselin, DL Rudland, H Xu, GM Wilkowski and BO Lydell. 2007. Probabilistic Fracture Mechanics Evaluation of Selected Passive Components - Technical Letter Report. PNNL-16625, Pacific Northwest National Laboratory, Richland, Washington. 
Singer RM, KC Gross, JP Herzog, RW King and S Wegerich. 1997. "Model-Based Nuclear Power Plant Monitoring and Fault Detection: Theoretical Foundations." In Proceedings of 1997 Conference on Intelligent System Applications to Power Systems, pp. 60-65. July 6-10, 1997, Seoul, South Korea. Korean Institute of Electrical Engineering.

Singer RM, KC Gross, RW King and S Wegerich. 1995. "A Pattern-Recognition-Based, Fault-Tolerant Monitoring and Diagnostic Technique." In Proceedings 7th International Symposium on Nuclear Reactor Surveillance and Diagnostics, pp. 13-14. June 19-23,1995, Avignon, France. OECD Nucl. Energy Agency.

Singer RM, KC Gross, M Walsh and KE Humenik. 1990. "Reactor Coolant Pump Monitoring and Diagnostic System." In 2nd International Machinery Monitoring and Diagnostic Conference. October 22-25, 1990, Los Angeles. CONF-901090-1.

Singh R. 2000. Three Decades of NDI Reliability Assessment. Report No. Karta-3510-99-01, Karta Technology, Inc., San Antonio, Texas.

Smeulers JPM, R Zeelen and A Bos. 2002. "PROMIS - A Generic PHM Methodology Applied to Aircraft Subsystems." In 2002 IEEE Aerospace Conference, pp. 3153-3159 Vol. 6. March 9-16, 2002, Big Sky, Montana. IEEE, Piscataway, New Jersey.

Smith CL, VN Shah, T Kao and G Apostolakis. 2001. Incorporating Aging Effects into PRA - A Feasibility Study Utilizing Reliability Physics Models. NUREG/CR-5632, U.S. Nuclear Regulatory Commission, Washington, DC.

Smith G, JB Schroeder, S Navarro and D Haldeman. 1997. "Development of a Prognostics Health Management Capability for the Joint Strike Fighter." In AUTOTESTCON '97, pp. 676-682. September 22-25, 1997, Anaheim, California. IEEE, Piscataway, New Jersey.

Sohn H, CR Farrar, FM Hemez, DD Shunk, DW Stinemates, BR Nadler and JJ Czarnecki. 2003. A Review of Structural Health Monitoring Literature: 1996-2001. LA-13976-MS, Los Alamos National Laboratory, Los Alamos, New Mexico.

Srivastava AN, RW Mah and C Meyer. 2009. NASA Integrated Vehicle Health Management Technical Plan Ver. 2.03. NASA, Aeronautics Research Mission Directorate, Aviation Safety Program [online document]. http://www.aeronautics; http://www.aeronautics.nasa.gov/nra pdf/ivhm tech plan c1.pdf.

Stevenson JD. 2006. "The Maintenance Rule." In Companion Guide to the ASME Boiler \& Pressure Vessel Code, Volume 2, Second Edition: Criteria and Commentary on Select Aspects of the Boiler \& Pressure Vessel and Piping Codes, pp. 503-515 ed: KR Rao. ASME Press, New York.

Strong E, JB Coble, S O'Reilly and JW Hines. 2011. "Feasibility of Prognostics for Transformer Remaining Useful Life Predictions." In MFPT: The Applied Systems Health Management Conference 2011, Enabling Sustainable Systems. May 10-12, 2011, Virginia Beach, Virginia. MFPT Society.

Strong E and JW Hines. 2012. "Comparison of Transient and Steady-State Order Analysis for Degraded Induction Motors." To be presented at the 8th International Conference on Nuclear Plant Instrumentation, Control, and Human-Machine Interface Technologies (NPIC\&HMIT), July 22-26, 2012, San Diego, California. 
Su L, G DeMare and M Nolan. 1993. "DARTS: An Enabling Technology for Concurrent. Engineering." In AUTOTESTCON '93 - IEEE Systems Readiness Technology Conference, pp. 383-388. September 2023, 1993, San Antonio, Texas. IEEE, Piscataway, New Jersey.

Su LP, M Nolan, G DeMare and DR Carey. 1999. "Prognostics Framework [for Weapon Systems Health Monitoring]." In AUTOTESTCON '99 - IEEE Systems Readiness Technology Conference, pp. 661-672. August 30-September 2, 1999, San Antonio, Texas. IEEE, Piscataway, New Jersey.

Su LP, M Nolan, G DeMare and B Norman. 2000a. "Prognostics Framework--UPDATE II." In AUTOTESTCON 2000 - IEEE Systems Readiness Technology Conference, Future Sustainment for Military Aerospace, pp. 497-504. September 18-20, 2000, Anaheim, California. IEEE, Piscataway, New Jersey.

Su LP, M Nolan, G DeMare and B Norman. 2000b. "Prognostics Framework: Software Design Tool." In 2000 IEEE Aerospace Conference. March 18-25, 2000, Big Sky, Montana. IEEE, Piscataway, New Jersey.

Suarez EL, MJ Duffy, RN Gamache, R Morris and AJ Hess. 2004. "Jet Engine Life Prediction Systems Integrated with Prognostics Health Management." In 2004 IEEE Aerospace Conference, pp. 3596-3602. March 6-13, 2004, Big Sky, Montana. IEEE, Piscataway, New Jersey.

Tang L, GJ Kacprzynski, K Goebel, J Reimann, ME Orchard, A Saxena and B Saha. 2007. "Prognostics in the Control Loop." In Artificial Intelligence for Prognostics - Papers from the AAAI Fall Symposium, pp. 128-135. November 9-11, 2007, Arlington, Virginia. American Association for Artificial Intelligence.

Tarantola A. 1987. Inverse Problem Theory: Methods for Data Fitting and Model Parameter Estimation. Elsevier Science BV.

Teo YH, WK Chiu, FK Chang and N Rajic. 2009. "Optimal Placement of Sensors for Sub-surface Fatigue Crack Monitoring." Theoretical and Applied Fracture Mechanics 52(1):40-49.

Tian Y, A Tamburrino, SS Udpa and L Udpa. 2003. "Time-of-Flight Measurements from Eddy Current Tests." In Review of Progress in Quantitative Nondestructive Evaluation, Vol. 22. July 14-19, 2002, Bellingham, Washington. American Institute of Physics, Melville, New York.

Tian Z, T Jin, B Wu and F Ding. 2011a. "Condition Based Maintenance Optimization for Wind Power Generation Systems Under Continuous Monitoring." Renewable Energy 36:1502-1509.

Tian Z, Y Zhang and J Cheng. 2011b. "Condition Based Maintenance Optimization for Multicomponent Systems " In Annual Conference of the Prognostics and Health Management Society. September 25-29, 2011, Montreal, Quebec. PHM Society.

Tikhonov AN and V Arsenin. 1977. Solutions of Ill-Posed Problems. Wiley.

Tipping PG, Ed. 2010. Understanding and Mitigating Ageing in Nuclear Power Plants. Woodhead Pubs, Cambridge. Woodhead Publishing Series in Energy.

Tobon-Mejia DA, K Medjaher and N Zerhouni. 2010. "The ISO 13381-1 Standard's Failure Prognostics Process through an Example." In 2010 Prognostics and System Health Management Conference (PHM '10), pp. 1-12. January 12-14, 2010, Macao, China. IEEE Computer Society, Piscataway, New Jersey. 
Trifanov A. 2007. "Status of Research Project - Incorporating Ageing Effects into PSA." In European Commission Workshop on Use of Probabilistic Safety Assessment (PSA) for Evaluation of Impact of Ageing Effects on the Safety of Nuclear Power Plants. November 15-16, 2007, Budapest, Hungary.

Turkcan E. 1985. "On-line Monitoring of a PWR for Plant Surveillance by Noise Analysis." Progress in Nuclear Energy 15:365-378.

Turkcan E, O Ciftcioglu and JP Verhoef. 1996. "New Technologies in Nuclear Power Plant Monitoring and Diagnosis." IAEA 621-SP-384.41. Presented at the Specialists' Meeting on Monitoring and Diagnosis Systems to Improve NPP Reliability and Safety, Gloucester, United Kingdon, May 14-17, 1996.

Udpa L. 2004. "Chapter 7, Signal and Image Processing for Electromagnetic Testing." In

Nondestructive Testing Handbook, Third Edition: Volume 5, Electromagnetic Testing, eds: SS Udpa and PO Moore. American Society for Nondestructive Testing, Columbus, Ohio.

Udpa L and SS Udpa. 1997. "Application of Signal Processing and Pattern Recognition Techniques to Inverse Problems in NDE." International Journal of Applied Electromagnetics and Mechanics 8:99-117.

Uhrig RE and JW Hines. 2005. "Computational Intelligence in Nuclear Engineering." Nuclear Engineering and Technology 37(2):127-138.

Unwin SD, PP Lowry, RF Layton Jr, PG Heasler and MB Toloczko. 2011a. "Multi-state Physics Models of Aging Passive Components in Probabilistic Risk Assessment." In International Topical Meeting on Probabilistic Safety Assessment and Analysis 2011 (PSA 2011) pp. 161-172. March 13-17, 2011, Wilmington, North Carolina. American Nuclear Society, La Grange, Illinois.

Unwin SD, PP Lowry and MY Yoyooka. 2010. "Component Degradation Susceptibilities a the Bases for Modeling Reactor Aging Risk." In Proceedings of the ASME 2010 Pressure Vessels \& Piping Division / K-PVP Conference (PVP2010). July 18-22, 2010, Bellevue, Washington. American Society of Mechanical Engineers, New York.

Unwin SD, PP Lowry, MY Yoyooka and BE Ford. 2011b. "Degradation Susceptibility Metrics as the Bases for Bayesian Reliability Models of Aging Passive Components and Long-Term Reactor Risk." In Proceedings of the ASME 2011 Pressure Vessels \& Piping Division (PVP2011). July 17-21, 2011, Baltimore, Maryland. American Society of Mechanical Engineers, New York.

Upadhyaya BR and F Li. 2011. Advanced Instrumentation and Control Methods for Small and Medium Reactors with IRIS Demonstration, Vol. 4: Dynamic Modeling, Sensor Placement Design, and Fault Diagnosis of Nuclear Desalination Systems. Report No. DE-FG07-07ID14895/UTNE/2011-6. Final Report prepared for the U.S. Department of Energy, University of Tennessee.

Upadhyaya BR, M Naghedolfeizi and B Raychaudhuri. 1994. "Residual Life Estimation of Plant Components." P/PM Technology June:22-29.

Upadhyaya BR, K Zhao and B Lu. 2003. "Fault Monitoring of Nuclear Power Plant Sensors and Field Devices." Progress in Nuclear Energy 43(1-4):337-342.

USDI. 2000. Transformer Maintenance, Facilities Instructions, Standards, and Techniques, Volume 331. FIST 3-30, United States Department of the Interior (USDI), Bureau of Reclamation, Washington, D.C. Available at http://www.usbr.gov/power/data/fist/fist3 30/fist3 30.pdf. 
USDI. 2003. Transformer Diagnostics, Facilities Instructions, Standards, and Techniques, Volume 3-31. FIST 3-31, United States Department of the Interior (USDI), Bureau of Reclamation, Washington, D.C. Available at http://www.usbr.gov/power/data/fist/fist3 31/fist3-31.pdf.

Usynin AV. 2007. A Generic Prognostic Framework for Remaining Useful Life Prediction of Complex Engineering Systems. PhD Thesis, University of Tennessee, Knoxville, Tennessee. Available at http://trace.tennessee.edu/utk graddiss/319.

Valentin R, M Osterman and B Newman. 2003. "Remaining Life Assessment of Aging Electronics in Avionic Applications." In Proceedings of Annual Conference on Reliability and Maintainability (RAMS 2003), pp. 313-318. January 27-30, 2003, Tampa, Florida. IEEE, Piscataway, New Jersey.

Venkatasubramanian V, R Rengaswamy and SN Kavuri. 2003a. "A Review of Process Fault Detection and Diagnosis: Part II: Qualitative Models and Search Strategies." Computers \& Chemical Engineering 27(3):313-326.

Venkatasubramanian V, R Rengaswamy, SN Kavuri and K Yin. 2003b. "A Review of Process Fault Detection and Diagnosis: Part III: Process History Based Methods." Computers \& Chemical Engineering 27(3):327-346.

Venkatasubramanian V, R Rengaswamy, K Yin and SN Kavuri. 2003c. "A Review of Process Fault Detection and Diagnosis: Part I: Quantitative Model-Based Methods." Computers \& Chemical Engineering 27(3):293-311.

Verma A, A Ibragimov and S Ramachandran. 2003. "Wavelet-Based Neural Net Application for Feature Detection and Classification." Neural, Parallel and Scientific Computations 11(1-2):19-40.

Vichare $\mathrm{N}$ and M Pecht. 2006. "Prognostics and Health Management of Electronics." IEEE Transactions on Components and Packaging Technologies 29(1):222-229.

Vichare N, P Rodgers, V Eveloy and M Pecht. 2004. "In Situ Temperature Measurement of a Notebook Computer - A Case Study of Health and Usage Monitoring of Electronics." IEEE Transactions on Device and Materials Reliability 4(4):658-663.

Villaran M and R Lofaro. 2010. Essential Elements of an Electric Cable Condition Monitoring Program. NUREG/CR-7000, BNL-NUREG-90318-2009, U.S. Nuclear Regulatory Commission, Washington, D.C.

Vipperman JS. 1999. "Structural Health Monitoring Applications Using Piezo-dielectric Effects." In The ASME International Mechanical Engineering Congress and Exposition: Adaptive Structures and Material Systems - 1999 (IMECE 1999), pp. 397-401. November 14-19, 1999, Nashville, Tennessee. ASME, Fairfield, New Jersey.

Vogel C. 2002. Computational Methods for Inverse Problems. Society for Industrial and Applied Mathematics (SIAM), Philadelphia.

Wacker JF, JI Friese, LO Casazza, JP McKinley, SL Petersen, KB Wagnon, GJ Lumetta, SE Bonde and JW Grate. 2007. FY06 Annual Report on the Progress and Path Forward for the NA-22 Funded Project PL06-AUT308-PD01: Automation of Ultra-Trace and Radiochemical Methods. PNNL-16527, Pacific Northwest National Laboratory, Richland, Washington. 
Wald A. 1945. "Sequential Tests of Statistical Hypotheses." Annals of Mathematical Statistics 16(2):117-186.

Walker MG. 2010. "Next Generation Prognostics and Health Management for Unmanned Aircraft." In 2010 IEEE Aerospace Conference. March 6-13, 2010, Big Sky, Montana. IEEE, Piscataway, New Jersey.

Walter R. 2006. Open Systems Architecture for Condition-based Maintenance (OSA-CBM) Primer. Penn StateUniversity/Applied Research Laboratory, The Boeing Company, and Machine Information Management Open Standards Alliance (mimosa). Technical Report.

Waltz EL and J Llinas. 1990. Multisensor Data Fusion. Artech House.

Wang M, AJ Vandermaar and KD Srivastava. 2002. "Review of Condition Assessment of Power Transformers in Service." IEEE Electrical Insulation Magazine 18(6):12-25.

Whisnant K, KC Gross and N Lingurovska. 2005. "Proactive Fault Monitoring in Enterprise Servers." In 2005 International Conference on Computer Design, CDES'05, pp. 3-10. June 27-30, 2005, Las Vegas, Nevada. CSREA Press, Bogard, Georgia.

Wilkinson M, F Spianto and M Knowles. 2006. "Towards the Zero Maintenance Wind Turbine." In 41st International Universities Power Engineering Conference (UPEC 2006), pp. 74-78. September 6-8, 2006, Newcastle upon Tyne, United Kingdom. IEEE, Piscataway, New Jersey.

Wilkinson MR, F Spinato and PJ Tavner. 2007. "Condition Monitoring of Generators Other Subassemblies in Wind Turbine Drive Trains." In IEEE Symposium on Diagnostics for Electric Machines Power Electronics and Drives (SDEMPED), pp. 388-392. September 6-8, 2007, Cracow, Poland. IEEE, Piscataway, New Jersey.

Wilkowski G, R Tregoning, P Scott and D Rudland. 2002. "Status of Efforts to Evaluate LOCA Frequency Estimates Using Combined PRA and PFM Approaches." In 28th MPA-Seminar. October 2002, Universitaet Stuttgart, Germany. Materials Testing Institute.

Willetts AJ and FV Ammirato. 1987. "Objectives and Techniques for Performance Demonstration of InService Examination of Reactor Pressure Vessels." In Performance and Evaluation of Light Water Reactor Pressure Vessels, pp. 79-86. June 28-July 2, 1987, San Diego, California.

WNA. 2011. The Economics of Nuclear Power. World Nuclear Association (WNA). Accessed July 3, 2012. Available at http://www.world-nuclear.org/info/inf02.html.

Wong S-M, FX Talbot, RM Latta and RP Correia. 1999. Lessons Learned from Maintenance Rule Baseline Inspections. NUREG-1648, U.S. Nuclear Regulatory Commission, Washington, D.C.

Xiang P, S Ramakrishnan, Y Cai, P Ramuhalli, R Polikar, S Udpa and L Udpa. 2001. "Automated Analysis of Rotating Probe Multi-Frequency Eddy Current Data from Steam Generator Tubes." International Journal of Applied Electromagnetics and Mechanics 12(3/4):151-164.

Xu D and W Zhao. 2005. "Reliability Prediction Using Multivariate Degradation Data." In Annual Reliability and Maintainability Symposium (RAMS 2005), pp. 337-341. January 24-27, 2005, Alexandria, Virginia. IEEE, Piscataway, New Jersey. 
Yan M, M Afzal, S Udpa, S Mandayam, Y Sun, L Udpa and P Sacks. 1998. "Iterative algorithms for electromagnetic NDE signal inversion." In Proceedings of the 3rd International Workshop on Electromagnetic Non-Destructive Evaluation II (ENDE (II), pp. 287-296. September 1997, Reggio Calabria, Italy. IOS Press, Lansdale, Pennsylvania.

Yang K and J Xue. 1996. "Continuous State Reliability Analysis." In 1996 Annual Reliability and Maintainability Symposium (RAMS '96), International Symposium on Product Quality and Integrity, pp. 251-257. January 22-25, 1996, Las Vegas, Nevada. IEEE, Piscataway, New Jersey.

Yang Q. 2004. Model-Based and Data Driven Fault Diagnosis Methods with Applications to Process Monitoring. Ph.D. Thesis, Case Western Reserve University, Cleveland, Ohio. Available at http://rave.ohiolink.edu/etdc/view?acc num=case1080246972.

Yang W, PJ Tavner and MR Wilkinson. 2009. "Condition Monitoring and Fault Diagnosis of a Wind Turbine Synchronous Generator Drive Train." IET Renewable Power Generation 3(1):1-11.

Yim J, SS Udpa, M Mina and L Udpa. 1996. "Optimum Filter Based Techniques for Data Fusion." In Review of Progress in Quantitative Nondestructive Evaluation (QNDE), Vol. 15, pp. 773-780. Plenum Press, New York.

Yim J, SS Udpa, L Udpa and M Mina. 1994. "Fusion of Ultrasonic and Eddy Current Images Using Radial Basis Function." In Proceedings of the 3rd Annual Midwest Electrotechnology Conference, pp. 10-14. April 1994.

Zaher A, SDJ McArthur and DG Infield. 2009. "Online Wind Turbine Fault Detection through Automated SCADA Data Analysis." Wind Energy 12:574-593.

Zetec. RevospECT. Zetec. Snoqualmie, Washington. Accessed March 15, 2012. Available at http://www.zetec.com/2010/07/revospect/.

Zhang QJ and KC Gupta. 2000. Neural Networks for RF and Microwave Design. Artech House.

Zimmerman AT, M Shiraishi, RA Swartz and JP Lynch. 2008. "Automated Modal Parameter Estimation by Parallel Processing within Wireless Monitoring Systems." Journal of Infrastructure Systems 14(1):102-113. 


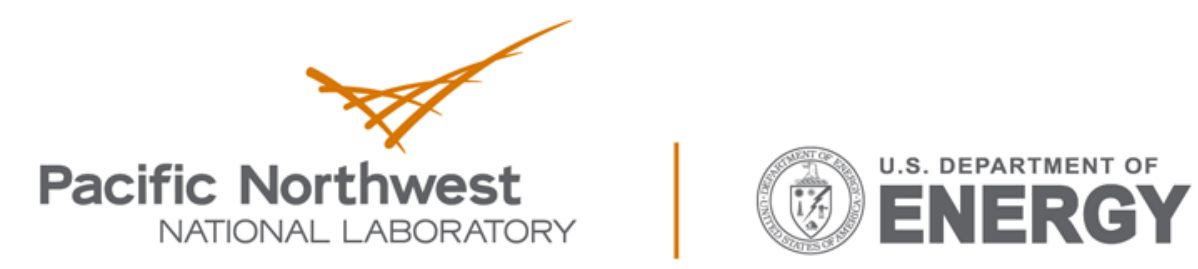

Proudly Operated by Battelle Since 1965

902 Battelle Boulevard

P.O. Box 999

Richland, WA 99352

1-888-375-PNNL (7665)

www.pnnl.gov 